
March 1938

\title{
AN ABSOLUTE ELECTROMETER FOR THE MEASUREMENT OF HIGH ALTERNATING VOLTAGES
}

\author{
By Herbert B. Brooks, Francis M. Defandorf, and Francis B. Silsbee
}

\begin{abstract}
An absolute electrometer of the attracted-disk type is described. It is suitable for the measurement of alternating voltages up to 275,000 volts effective value with an accuracy of a few hundredths of a percent. A set of equally spaced coaxial guard hoops, maintained at equally spaced potentials, serves to produce a uniform field at the disk in spite of the large separation $(110 \mathrm{~cm})$ required to avoid sparkover at the high operating voltage. Formulas are derived by which corrections can be applied for any deviation of the individually measured hoop potentials from the ideal equal spacing.

The disk hangs from one arm of a delicate balance which serves to measure the force of attraction. Light reflected from a mirror carried by the balance beam serves to magnify its motion and to indicate to the operator at a safe distance when a condition of equilibrium is reached. It also serves to indicate the height of the disk relative to its surrounding guard ring. The scale reading corresponding to the ideal coplanar condition is obtained from auxiliary measurements made with a pair of special microscopes adapted for measuring distances in the line of sight by a calibrated focus adjustment.

The change in the attractive force as the disk moves away from the coplanar position has been measured and compared with that calculated theoretically. The effects of this change on the sensitivity and on the stability of the balance are worked out in detail.

Trials of this instrument under various conditions, both normal and with certain adverse influences exaggerated, over the range from 10,000 to 100,000 volts, indicate that the probable error of values obtained with it is about 0.01 percent, and that this error will not be greatly increased when the instrument is used at 275,000 volts.
\end{abstract}

\section{CONTENTS}

I. Introduction

II. General description

III. Stability and sensitivity

IV. Formulation of the working equation

V. Precision and accuracy

VI. Experimental tests

1. Electrical discharges.... 270

2. Tilt of disk and of lower plate...

3. Eccentricity of disk in aperture

4. Width of gap

5. Flatness of disk and of guard ring

6. Ventilating holes....

7. Temperature

8. Guard-hoop potentials.

9. Flatness of lower plate.... 285

10. Vibration

VII. Mechanical details

1. Main structure

2. Lower plate

3. Disks and damping chamber

4. Balance

5. Potential divider and hoops 
VIII. Details of operation

1. Weighing

2. Measurement of coplanarity

3. Gaging

4. Measurement of diameters

5. Survey of the disk

6. Measurement of hoop-potential distribution

IX. Mathematical details_...

1. Correction for nonuniformity of hoop-potential distribution... 300

2. Sources of error in Fourier coefficients, $G_{k}{ }^{\prime} \ldots \ldots \ldots \ldots$

3. Variation of force with height of disk

$\mathrm{X}$. Conclusions and discussion

XI. List of principal symbols

\section{INTRODUCTION}

The growth of the electrical industry has been marked for many years by a continued increase in the voltage at which electrical energy is transmitted. This is the natural result of the greater economy of high-voltage transmission for long distances and large amounts of power. The art of measuring alternating voltages and of measuring power and energy at high voltages has kept pace with this growth by extending further and further the methods and types of apparatus originally developed for more moderate voltages.

It seemed desirable to develop an independent method for measuring high voltages with an accuracy approaching 0.01 percent, which would afford a check on the methods at present in use. Such a method should provide a safeguard against any constant and unrecognized errors which tend to creep into the measurements of quantities which are widely different in magnitude from their basic units. Such an independent method for measuring high voltages was felt to be particularly desirable at the National Bureau of Standards, because one function of this Bureau is to check instrument voltage transformers and other apparatus used throughout this country in the commercial measurement of high voltages.

The precise methods in common use for the measurement of effective values of high alternating voltages involve a stepping-up by a factor of about 100,000 from the electromotive force of a standard cell to the voltage to be measured, together with a transfer from direct to alternating current by an instrument of the electrodynamic type. It was felt that the greatest possible contrast to this type of method would be obtained by using an absolute electrostatic instrument to which the entire potential difference to be measured could be directly applied. The attracted-disk type of instrument was adopted because in it the maximum permissible electric field is utilized most effectively, and because it is most adaptable to shielding from external disturbances.

This type of instrument was originally suggested by Sir William Snow Harris ${ }^{1}$ in 1834. His rudimentary device, shown in figure 1, consisted of a metal disk $d$ hung from one arm of a balance and a second disk $a$ supported coaxially below the suspended disk. The force of attraction between the disks is proportional to the square of the potential difference between them. Because of the crowding of the electric charges toward the edges of the disk, and because of the

\footnotetext{
1 Sir William Snow Harris. On a new electrometer, and the heat excited in metallic bodies by voltaic electricity. Trans. Roy. Soc. Edinburgh 12, 206 (1834).
} 




Figure 1.-Attracted-disk electrometer of Sir William Snow Harris, 1834. 
forces exerted by neighboring objects, voltage measurements with the instrument in this form have relative significance only.

To remove this limitation of the Harris electrometer, and to develop it into an instrument suitable for accurate measurements, Lord Kelvin , ${ }^{2}$ about the year 1870 , surrounded the attracted disk by a fixed "guard ring" electrically connected to the disk, the flat lower surface of the guard ring being in the same plane as the lower surface of the disk. He also increased the diameter of the lower plate to equal the outside diameter of the guard ring. By making this diameter and that of the lower plate several times as great as the separation between these parts, Kelvin greatly reduced the effect of nonuniformity of charge at their edges on the force acting on the disk.

The simple theory of the Kelvin guard ring electrometer considers the disk, guard ring, and lower plate as plane parallel surfaces, and makes further assumptions, some of which, of a geometrical nature, are as follows:

1. That the lower surface of the disk and that of the guard ring are in the same plane.

2. That the clearance between the disk and the guard ring is zero, although the disk is free to move vertically.

3 . That the common diameter of the guard ring and of the lower plate is so great in comparison with the distance between these parts that any further increase in this diameter would produce an inappreciable effect upon the electric field acting on the disk and therefore upon the force acting on the disk.

With these assumptions, it may be shown that for an effective difference of potential between the disk and the lower plate of $V_{s}$ electrostatic units the electric force on the disk is

$$
F=\frac{V_{s}^{2} A}{8 \pi b^{2}} \text { dynes, }
$$

where $A$ is the area of the disk and $b$ is the separation between it and the lower plate.

When the condition in assumption 1 is not realized, that is, when the disk either recedes into or protrudes from the guard ring, the electric charge ceases to be uniformly distributed over the surface of the disk and eq 1 must be extended to include a correction for the lack of coplanarity.

The condition in assumption 2 cannot be realized. There must be a gap between the disk and the guard ring. The effect of this gap, when the lower surface of the disk and that of the guard ring are coplanar, has commonly been allowed for by regarding the effective radius of the disk as exceeding the actual radius by one-half of the width of the gap.

The assumption made in condition 3 is not difficult to realize when the voltage to be measured is only a few kilovolts. It is obvious from eq 1 that in any event, to make the electric force to be measured large, the separation $b$ should be small. For any given voltage, however, there is a minimum separation ${ }^{3}$ below which sparkover will occur. For the high voltages now in commercial use (220 kilovolts and higher), this minimum value of $b$ is so large as to make it impracticable to use a

\footnotetext{
${ }^{2}$ Lord Kelvin, Papers on Electrostatics and Electromagnetism, Sect. 360, 2d ed. p. 287 (Macmillan \& Co., London, 1884).

3 Instruments of smaller dimensions immersed in compressed gas have been used successfully by Palm (Z. tech. Physik 1, 137 (1920); 14, 390 (1933)) for high-voltage measurement and give large forces, but operation in the open seemed to us to offer greater convenience for studying the effect of various sources of error.
} 
guard ring and a lower plate large enough to satisfy assumption 3 . When the present investigation was begun, search was therefore made for some practicable method by which the electric force on the disk could be made to have the same value, to a sufficient approximation, as it would have if the common diameter of the guard ring and the lower plate were four or five times as great as the separation between them. Such an expedient ${ }^{4}$ was found in a system of equally spaced coaxial "guard hoops" which enclose the cylindrical space between the guard ring and the lower plate. In operation, these hoops are maintained at equally spaced potentials, intermediate between those of the guard ring and of the lower plate.

In addition to the three geometrical assumptions just discussed, the use of an actual instrument of this type involves the further assumptions that in its construction the theoretical conditions, such as flatness and parallelism of surfaces, squareness of edges, equality of spacing of the hoops and of their potentials, etc., have been realized with sufficient exactness, and also that spurious effects, such as an electric wind from a brush discharge, are not present to a significant extent. In the present work wherever possible, as for example in the case of the hoop potentials, the departures of these various conditions from the ideal have been the subject of auxiliary measurements the results of which, when substituted in theoretically derived equations, give the corrections needed to allow for the departures. In the case of other conditions, such as coplanarity and flatness, our procedure has been to make observations under conditions definitely on either side of the ideal and to obtain the final result by interpolation. For the remaining and more numerous class of conditions, such as lack of parallelism, eccentricity, etc., we have performed check experiments with the disturbing condition present to an exaggerated degree, and from the smallness of the resulting effect on the indication of the instrument we have felt justified in neglecting the error present under normal operating conditions.

An absolute electrometer embodying these principles has accordingly been designed and built at the National Bureau of Standards for measurements up to 275,000 volts. It is the purpose of the present paper to describe this instrument in detail and to give the fundamental basis on which its indications depend and experimental data showing its possibilities and limitations. The comparison of results obtained with it and those obtained by the conventional method of measuring high voltages is treated in another paper. ${ }^{5}$

\section{GENERAL DESCRIPTION}

The general appearance of the electrometer with its capacitance potential divider is shown in figure 2 . It is about $1 \mathrm{~m}$ in diameter and $3 \mathrm{~m}$ high. The lower plate is adjustable in height to give separations up to $110 \mathrm{~cm}$.

The fundamental features of the instrument are shown schematically in the center of figure 6 . It is evident that ideally the disk forms the central part of the upper plane equipotential boundary of a cylindrical space, the lower plate forms the other parallel equi-

- Chester Snow. Effect of clearance and displacement of attracted disk, and also of a certain arrangement of conducting hoops upon the constant of an electrometer. BS J. Research 1, 514 (1928) RP17.

$\checkmark$ Francis B. Silsbee and Francis M. Defandorf. A transformer method for measuring high alternating voltages and its comparison with an absolute electrometer. J. Research NBS 20, 317 (1938) R P1079. 


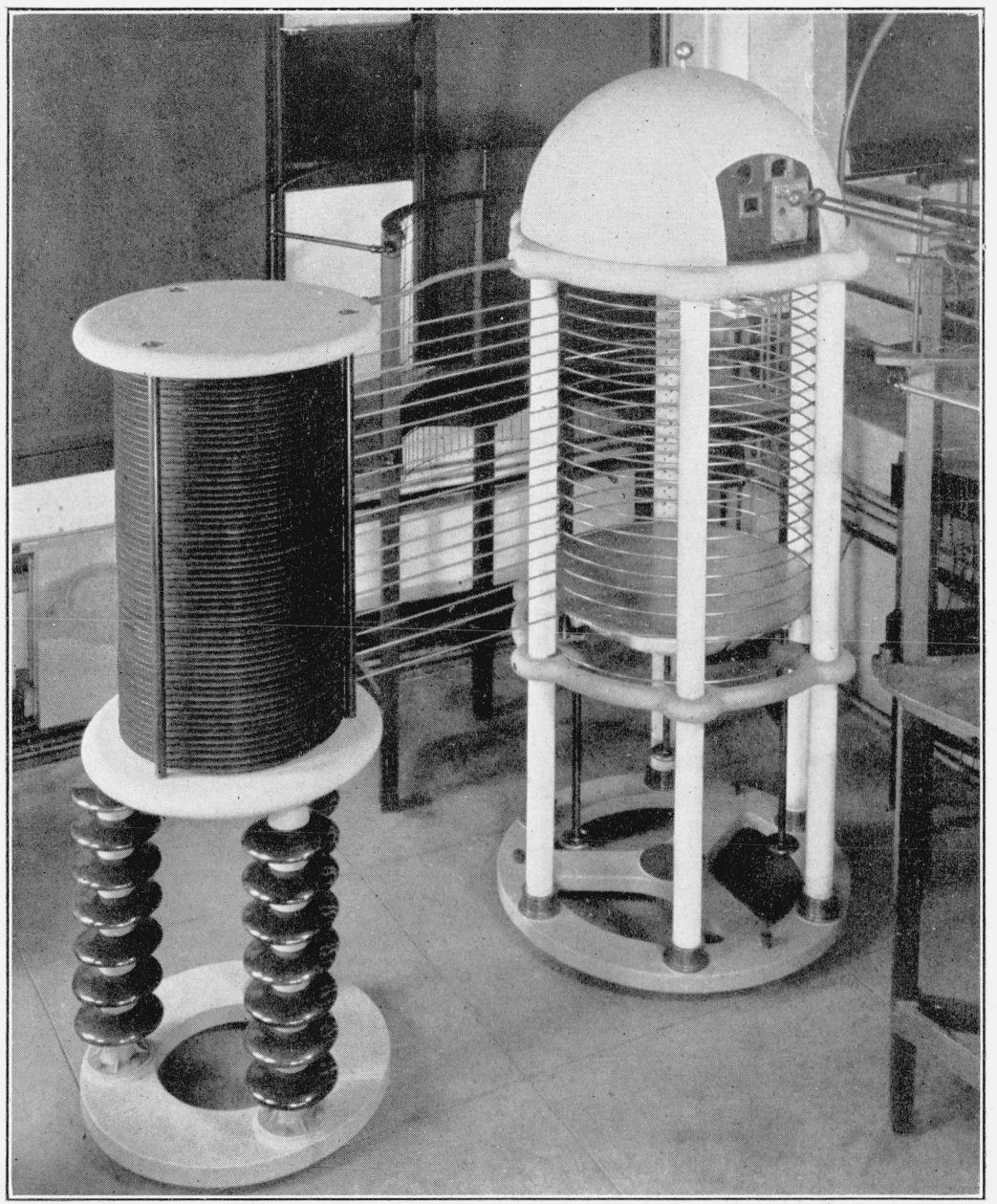

Figure 2.-Electrometer (right) and capacitance potential divider (left). Hoops are here shown spaced at $6 \mathrm{~cm}$ instead of at the normal $2 \mathrm{~cm}$. 


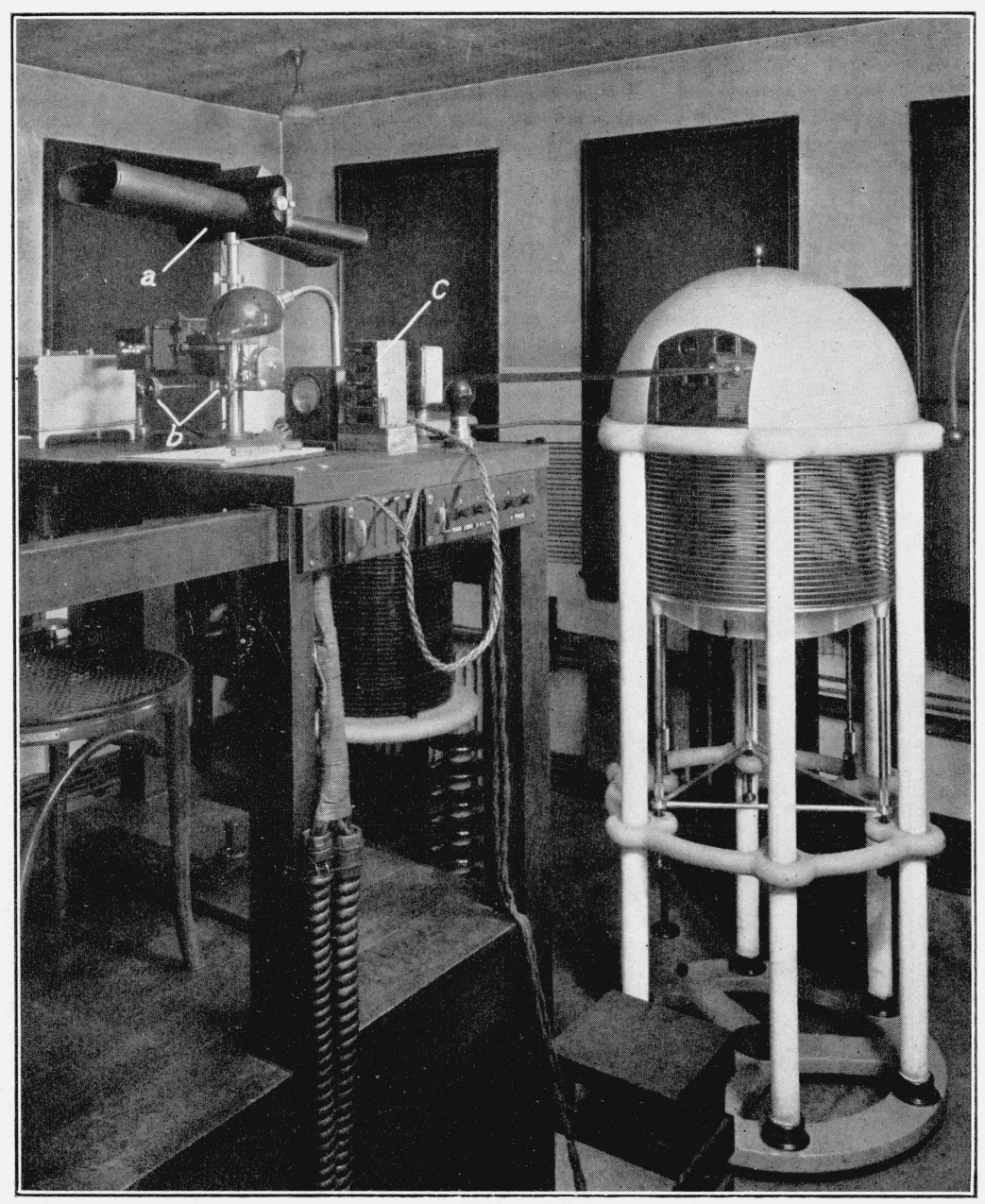

FIGURE 3.-Electrometer (right) and observer's control station (left).

$a$, Mounting for ground-glass scale; $b$, control-rod handles; and $c$, voltage-control box. 
potential boundary, while the cylindrical boundary surface is forced by the charged hoops to have a potential gradient which is uniform (except for a slight ripple near the hoops which has a negligible effect at the disk). The dome-shaped metal cover protects the balance and the upper surface of the disk from electrostatic forces and also from air currents and dust.

The upper plate, disk, and dome are at a high potential above ground when the electrometer is in use. To assure the safety of the operator the mechanisms for arresting and controlling the balance are operated by long insulating rods and the balance is read by an optical lever system.

In use, the counterpoise is adjusted for equilibrium. Known weights are then removed from a pan on the disk arm. Voltage is applied and adjusted to restore equilibrium by producing an electrostatic force equal to the weight just removed. The motion of the balance is nearly critically damped by the piston action of the disk, and the observer adjusts for an exact balance at the scale zero instead of using the more customary procedure of observing the successive turning points in the swings of an undamped balance.

A more detailed idea of the construction of the electrometer can be obtained from figure 27 , which will be found facing p. 314 . This figure is in effect a vertical cross section through the axis of the instrument, but many of the smaller parts are drawn to an exaggerated scale and others, particularly parts of the optical system, have been so shifted in azimuth as to more clearly indicate their function.

The attracted disk (1) hangs in an aperture in the guard ring, which consists of the upper plate (4) and the bushing (3) which fits into it. The disk is electrically connected to the guard ring by gold ligaments (17) which conduct the charging current of the disk around the agate knife-edges. The guard ring is supported by six hollow pillars (36) of fused silica which rest on the base ring (43). An intermediate ring (42), rigidly attached to the silica pillars, carries the three rods (38) which support the lower plate (35) and also carries the jack screws (41) of coarse pitch which are used only in making large changes in the height of the lower plate. The support rods (38), of invar, which support the lower plate when the instrument is in use, rest on three micrometer screws (40) of fine pitch. These screws are used in the final adjustment of the lower plate to the proper level.

The silica pillars also support the guard hoops (31), each of which rests on three horizontal wooden pegs (32) inserted in equally spaced holes drilled in three of the six silica pillars. The leads connecting each hoop to the appropriate tap in the potential divider are seen in figure 2.

The more essential features of the balance mechanism are shown schematically and enlarged above the guard ring in figure 27 . The weights, aggregating 0.1 to $2.5 \mathrm{~g}$, rest on a weight pan (16) which can be lowered onto the stirrup when the instrument zero is to be read, or raised by the lifter (15) when voltage is to be measured. The counterweight is at (20) and the fine gold chain (21), one end of which is attached to the beam, while the other can be moved vertically by the arm, serves as a fine adjustment of the mass on the counterweight arm of the balance.

The stabilizing arm (22), on which may be placed weights such as that shown, provides the stabilizing effect required to offset the 
inherently unstable action which would otherwise result from the variation of the electrostatic force. Under extreme conditions a stabilizing mass of $15 \mathrm{~g}$ at a distance of $12 \mathrm{~cm}$ below the central knifeedge is required. Arrestments are provided: (a) For lifting the beam off its central knife-edge; (b) for lifting the stirrup, which carries the disk and weight pan, off the end knife-edge; and (c) for clamping the stem of the disk, while the weight pan is being raised or lowered by its lifter. This procedure of clamping the stem (clamp 12) obviates any relative shift of the knife-edges with respect to their bearings between the time when the voltage is measured with the weights removed and the time when the zero is observed with the weights on the stirrup and the voltage removed. The clamp and weight lifter, as well as the "chainomatic" arm, can be controlled through long insulating rods from the observer's station. (See fig. 3.)

To indicate the position of the beam and thus the height of the attracted disk, the mirror (18) attached to the balance beam reflects the image of an illuminated reticle (27) onto the ground-glass electrometer scale at the observer's station. This optical method is equivalent to the use of a pointer $5 \mathrm{~m}$ long. For clearness, in figure 27 the prism (13) has been drawn in a position turned $90^{\circ}$, about a vertical axis, from that in which it is actually located. Its position in the electrometer is such that a tilt of the beam through a vertical angle produces a horizontal motion of the image. A second image of the illuminated scale is formed by reflection from a second mirror (19) rigidly supported near the beam mirror. The position of the beam image relative to this fiducial image gives a true indication of the position of the beam and disk and is unaffected by any motion of the observer's platform and its scale relative to the electrometer proper.

Four bushings (3) have been provided, each of which accurately fits the central opening in the upper plate and reduces it to a diameter suitable for a disk of $10,12,14$, or $16 \mathrm{~cm}$ diameter, respectively. To permit the required accurate shaping of the edge and of the flat surface at the important region adjacent to the disk, a ring (2) of stainless steel $20 \mathrm{~cm}$ in outside diameter is inserted at the inner edge of each bushing.

The vertical separation between the guard ring and the lower plate is measured by gage rods (33) inserted through holes in the bushing, the relative heights of the top of the rod and of the block (6) being shown by a sensitive surface gage (5).

In order to correlate readings on the electrometer scale with the height of the disk relative to the guard ring, auxiliary measurements of this height are made for some particular scale reading, using the coplanarity microscope (29). The optical system of one of these instruments is indicated schematically in figure 27 and is described in more detail in section VIII-2. For this measurement, before and after each series of weighings, a mounting containing a pair of such microscopes is clamped to the under side of the guard ring. These microscopes are focused alternately on the lower surface of the disk and on that of the guard ring, and the difference in height is derived from the observed difference in focus adjustment. 


\section{STABILITY AND SENSITIVITY}

The functioning of the electrometer depends essentially on a balance between the electrostatic and the mechanical forces acting on the disk, and hence its sensitivity and even its stability are vitally affected by the manner in which these forces vary with the position of the disk. A downward displacement of the disk from its normal position will increase the force of attraction; first, because of the increase in the electric gradient with the decrease in the distance to the lower plate, and second, because of the tendency for electric charge to redistribute itself, its density increasing on the protruding disk at the expense of that on the adjacent portions of the guard ring. The first, and more obvious, effect varies inversely as the separation between the guard ring and the lower plate, and becomes negligible at the widest spacings. The second effect, however, though less evident on first consideration, is so large as to predominate except at very small separations, and increases slowly with separation. The resultant effect is consequently very appreciable at all separations. The attractive force $F$ for a given (constant) voltage may be expressed in the form

$$
F=F_{0}(1-f h),
$$

where $F_{0}$ is the force when the disk and the guard ring are coplanar, $h$ is the height of the disk above the plane of the guard ring, and $f$ is a coefficient which depends somewhat on $h$ and on the proportions of the instrument. Under most conditions used in the present work, the force changes between 10 and 20 percent for 1-mm displacement of the disk so that $f$ in cgs units lies between 1 and 2 . This large value of $f$, and hence rapid change in the attractive force with displacement, has two effects on the operation of the electrometer. First, it makes it necessary to determine with high accuracy the true zero (i. e., the position of the spot of light for which the disk and the guard ring are truly coplanar); and second, it reduces the stability ${ }^{6}$ of the balance to such an extent that the moving system would be unstable under most conditions if some stabilizing device were not added.

Considering first the precision required in measuring $h$, we find from eq 2 that, for a constant voltage

$$
\frac{1}{F} \frac{d F}{d h}=-f \text { approx. }
$$

For the scale magnification by the optical lever of 80 and for the typical value of 1.6 for $f$, a precision of 1 percent in voltage requires that the height $h$ of the disk be known to $1 / 8 \mathrm{~mm}$ and that the scale be read merely to $1 \mathrm{~cm}$. On the other hand, for a precision of 0.01 percent in voltage the scale must be read to $0.1 \mathrm{~mm}$ and the height of the disk relative to the guard ring must be known to about $1 \mu$. Except at very small separations, this latter requirement is materially more exacting than the precision required in measuring the vertical separation $c$ between the guard ring and the lower plate. For example, when the separation is $50 \mathrm{~cm}$, the height of the disk above the guard ring must be known 25 times as precisely as its height above the lower plate.

\footnotetext{
- This tendency toward instability was discussed by Kelvin and more particularly by Abraham and Lemoine, J. phys. [3] 4, 463 (1895).
} 
For a precision of 0.01 percent the permissible error in the measurement of $h$ is in fact less than the unavoidable bulging and irregularities in the lower surface of the disk, and the question arises, which parts of this surface should be measured to determine the mean values used for the separation and for the displacement. Except when the separation is small, the major part of the term involving $f h$ results from the increase or diminution of electrostatic flux near the edge of the disk.

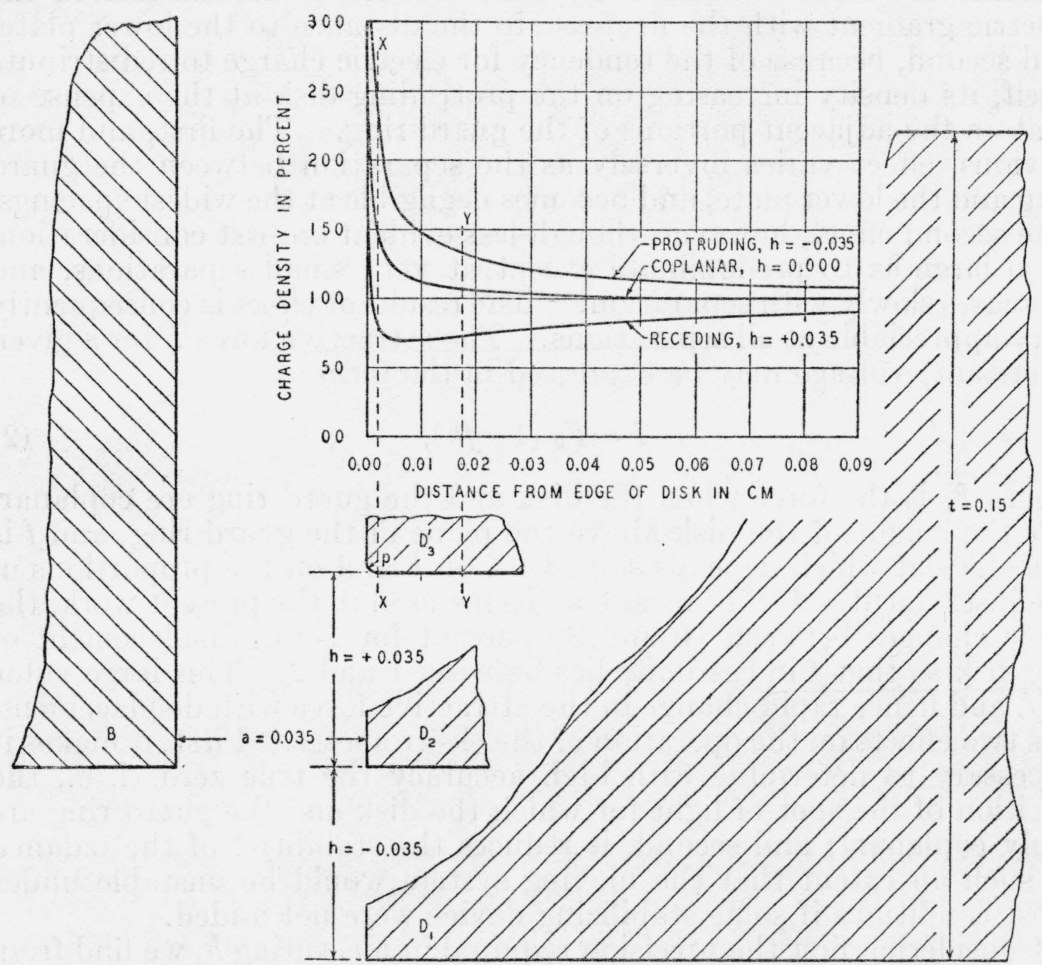

FIGURE 4.-Curves of theoretical distribution of charge on the disk near its edge, and magnified diagram showing relation of parts near the gap.

Ordinates give the charge density for a disk $10 \mathrm{~cm}$ in diameter with a gap $0.035 \mathrm{~cm}$ wide in percentage of the charge density at its center. The curves marked "protruding" and "receding" are for vertical displacements of $0.035 \mathrm{~cm}$ of a normal (i. e., square-cornered) disk from its "coplanar" position.

To the same magnified scale as the abscissas, this figure also shows: (1) The relative positions of the bushing $B$, and disk with the gap width $a=0.035 \mathrm{~cm}$; (2) displacements, $h=0.035 \mathrm{~cm}$ of the disk from its "coplanar" position at $D_{2}$ to "protruding" at $D_{1}$ and "receding" at $D_{3}$ shown, however, in this case with the edge rounded; (3) the actual disk thickness, $t=0.15 \mathrm{~cm}$; (4) the center of curvature $p$ on line $X X$ with the edge of the disk at $D_{\mathbf{3}}$ shown rounded as in test of table 2 , set $C$; and (5) intersection of line $Y Y$ with lower surface of disk which is the average position both of the center of the portion of the disk sighted when the coplanarity microscopes are used, and of the finger of the optical indicator when used in surveying the rim of the disk.

The graphs of figure 4 show the theoretical distribution of charge near the edge of the electrometer disk. These graphs are based on a paper by Snow. ${ }^{7}$ His equation 8 has here been applied to the case of a 10 -cm disk with a separation of $28 \mathrm{~cm}$, and a gap between the disk and the edge of the opening in the guard ring of $0.035 \mathrm{~cm}$, which is somewhat larger than that normally used. The ratio of the density of charge at any point on the surface to that at the center of the disk has been

\footnotetext{
${ }^{7}$ Chester Snow. Effeci of clearance and displacement of attracted disk, and also of a certain arrangement of conducting hoops upon the constant of an electrometer. BS J. Research 1, 513 (1928) RP17.
} 
plotted as ordinate against distance inward from the edge of the disk as abscissa, for three different positions of the disk. It is evident from these graphs that the major part of the excess or deficiency of charge, as compared with the ideal case in which there is no gap or displacement, occurs mainly on a peripheral belt a few millimeters wide around the circumference of the disk. Hence $h$ should be taken as the mean vertical displacement between the adjacent edges of the disk and the guard ring, and the term involving $f$ should vanish when $h$ is zero.

Hitherto we have considered only a perfectly plane disk, which cannot be exactly realized in practice. If the actual disk were indented so that on the average its surface were higher than its rim by an amount $h_{d}$, then, when the edges were coplanar the mean distance from the disk to the lower plate would be $c+h_{d}$ and the force would be expected to be less than that on a plane disk by the factor $c^{2} /\left(c+h_{d}\right)^{2}$. Hence the use of the quantity $c+h_{d}$ in place of $c$ for the separation, as is indicated in the factor affecting the right-hand member of eq 12 below, would be expected to correct for such a deviation of the surface of the disk from an ideal plane, if the resulting increase in distance were the only effect. As is shown below, however (see fig. 9), this procedure is not sufficient and a further correction, determined from experiments using nonplanar disks as described in section VI-5, must be applied as indicated by the term $\varphi_{1}\left(h_{d} / r_{d}\right)$ in eq 12 .

The second effect of the rapid variation of attractive force with disk height, namely, the effect on the stability of the balance, is best treated in connection with the following general consideration of the behavior of the balance during the weighing procedure. In any balance certain factors, such as the height of the central knife edge above the center of gravity of the beam and above the line joining the end knife-edges, introduce a restoring moment which increases approximately in proportion to the tilt of the beam and opposes the moment caused by the unbalance of the weights. In this electrometer two other restoring moments contribute to the stability of the balance. One is produced by the "chainomatic" device and is proportional to the tilt, to the mass of the chain per unit length, and to the square of the distance from the central knife-edge to the point of attachment of the chain. The other, and much the largest moment of all, is produced by stabilizing weights which may be attached as desired at definite distances along an aluminum rod which extends downward from the center of the beam. The total effective mechanical "stiffness" (i. e. restoring moment per unit angle) resulting from all these effects may be designated by $P$, and expressed in dyne-centimeters per radian. The turning moment produced by these stabilizing effects will be proportional to the angular tilt of the beam away from some particular (though unknown) position at which their resultant is zero. Let the reading of the spot of light on the electrometer scale for this hypothetical position be $S_{D}$. Then, when the scale reading has some other value, $S$, the increase in tilt in radians will be $\left(S-S_{D}\right) / L$, where $L$ is the effective pointer length ${ }^{8}$ of the optical system, and the torque tending to reduce this tilt will be $P\left(S-S_{D}\right) / L$.

The process of weighing consists essentially in two operations. In the first, with no voltage applied to the instrument, a mass $M_{F}$,

\footnotetext{
${ }^{8}$ This effective pointer length is approximately twice the distance from the mirror to the scale but is somewhat less than this, because the axis of rotation is not parallel to the mirror.
} 
consisting of the weights and the weight pan, is added to the mass $M_{d}$ on the disk arm of length $l_{d}$ of the balance, and this total is balanced to give a scale reading $S_{o}$ by the counterpoise of mass $M_{t}$ acting with an $\operatorname{arm} l_{t}$ and by the effective mass $M_{c}$ of the chain at an arm $l_{c}$. This effective mass is that borne by the balance when the reading is $S_{D}$. There may also be a force $A_{0}$ acting upward on the disk at this time as a result of air currents. Equating moments about the central knife-edge leads to the equation

$$
\left(M_{F}+M_{d}\right) g l_{d}-A_{o} l_{d}=M_{t} g l_{t}+M_{c} g l_{c}+P\left(S_{o}-S_{D}\right) / L
$$

In the second operation $M_{p}$ is removed, but the electric force $F$ is acting on the disk, and the air force may have a new value $A_{v}$. The voltage applied to the instrument is alternating and the instantaneous electrostatic force pulsates sinusoidally, at double the frequency of the voltage, between zero and a maximum value and is proportional at each instant to the square of the instantaneous voltage. The mean value of the force over a cycle is therefore equal to the constant force which would be produced by a constant voltage, the value of which is equal to the effective (root-mean-square) value of the actual alternating voltage. The period of swing of the balance is so very great (several seconds) in comparison with the period $(0.0083 \mathrm{sec})$ of the force produced by a 60-cycle voltage, that the amplitude of the forced oscillations is very small and the instrument may be taken as an indicator of the effective value of the applied voltage. Considering therefore only the constant mean force $F$, the equation with voltage applied is

$$
\left(M_{d} g+F\right) l_{d}-A_{v} l_{d}=M_{t} g l_{t}+M_{c} g l_{c}+P\left(S_{0}-S_{D}\right) / L
$$

Combining eq 3 and 4 gives for the attractive force

$$
F=M_{F} g+\left(A_{v}-A_{o}\right)+P\left(S_{o}-S_{o}\right) / L l_{d}
$$

If the scale reading during the measurements with the coplanarity microscopes was $S_{m}$, and if these measurements showed the average rim of the disk to be $h_{m}$ centimeters above the average rim of the guard ring, then at the time of weighing, the rim of the disk was

$$
h=h_{m}-\left(S_{v}-S_{m}\right) l_{d} / L
$$

centimeters above the rim of the guard ring.

Inserting this value and that for $F_{o}$ (from eq 51 section IX 3 ) into the expression (eq 2) for the force and denoting by $q$ the ratio $L / l_{d}$ by which the motion of the disk is magnified at the scale, gives

$$
F=\frac{V_{s}^{2} r_{m}{ }^{2}}{8 c^{2}}\left[1-f h_{m}+f\left(S_{v}-S_{m}\right) / q\right]
$$

Here the factor outside the bracket is the force for the coplanar position and the bracket gives the correction factor which allows for the change in force with height of the disk relative to the guard ring. Equation 7 may be combined with eq 5 above; and the result solved for $V_{s}$. This gives

$$
V_{s}=\frac{2 c \sqrt{2 M_{r} g}}{r_{m}}\left[\frac{1+\frac{A_{v}-A_{o}}{M_{r} g}+\frac{P\left(S_{v}-S_{o}\right)}{M_{r} g q l_{d}^{2}}}{1-f h_{m}+f\left(S_{0}-S_{m}\right) q}\right]
$$


When the instrument is operated at its normal undeflected position, the differences $\left(S_{v}-S_{o}\right)$ and $\left(S_{v}-S_{m}\right)$, as well as the air forces and the term $f h_{m}$, are relatively small and the polynomials containing them will give a rapid convergence when expanded by the binomial theorem. This procedure and the insertion for $r_{m}$ of its value $\left(r_{b}+r_{d}\right) / 2$ gives to a very close approximation

$$
V_{s}=\frac{4 c \sqrt{2 M_{F} g}}{r_{b}+r_{d}}\left[1+\frac{A_{v}-A_{0}}{2 M_{F} g}+\frac{f h_{m}}{2}-\frac{f\left(S_{v}-S_{m}\right)}{2 q}+\frac{P\left(S_{v}-S_{0}\right)}{2 q l_{d}{ }^{2} M_{F} g}\right]
$$

It follows from eq 7 that a lowering of the disk corresponding to an increase $\Delta S_{v}$ in reading causes a relative increase in the attractive force of $f \Delta S_{v} / q$. The corresponding increase in the restoring force is $P \Delta S_{v} / q l_{d}^{2} M_{F} g$. Unless the latter exceeds the former the balance will be unstable. The condition at which the balance is at the threshold of stability corresponds to

$$
P=f l_{d}^{2} M_{F} g
$$

and $P$ must always be adjusted to a value somewhat greater than this.

It follows from eq 8 that the relative error in voltage $\Delta V_{s} / V_{s}$ resulting from an error $\Delta S_{0}$ in reading the zero with no voltage applied, is

$$
\frac{\Delta V_{s}}{V_{s}}=-\frac{P \Delta S_{0}}{2 q M_{F} g l_{d}^{2}}
$$

Since $P$ cannot be less than the value giving stability, this sensitivity when reading the zero cannot be better than $\Delta V_{s} / V_{s}=f \Delta S_{0} / 2 q$. For $f=1 \mathrm{~cm}^{-1}$ and $q=80$ an error of $1 \mathrm{~mm}$ in reading $S_{0}$ gives an error of 0.06 percent in the measurement of voltage.

On the other hand, the relative error in voltage resulting from an error $\Delta S_{v}$ in balancing the instrument while voltage is applied is, assuming $f$ to be constant,

$$
\frac{\Delta V_{S}}{V_{s}}=\frac{1}{2 q}\left\{\frac{P}{l_{d}^{2} M_{F} g}-f\right\} \Delta S_{v}
$$

and can be made as small as desired by adjusting $P$ to be only very slightly greater than the critical value. Since the process for determining $S_{v}$ is substantially the same as that for $S_{0}$, the only complication being unsteadiness caused by fluctuations in voltage, there is little to be gained by making $P$ less than 1.1 times $f l_{d}{ }^{2} M_{F} g$. The period of swing is then not excessively long and the error $\Delta V_{s}$ from a given $\Delta S_{0}$ is only 10 percent worse than the unavoidable minimum.

The stability coefficient $P / 2 g q l_{d}{ }^{2}$ can be denoted by the single letter $\gamma$ and can be measured experimentally by determining the sensitivity of the balance with no voltage applied. In terms of this quantity the criterion for stability becomes $2 \gamma / M_{F} \geqq f / q$.

It is of interest to note that the tendency toward instability is so great that we are obliged to use values of the stifiness $P$ which are from 7 to 50 times as great as that which would be feasible if the electrical action tending to instability did not exist. Hence a proportional and very large loss of sensitivity is incurred.

The steadiness of the a-c voltage and the period of the balance are such that by careful manipulation we can obtain experimental scale- 
law curves even in the unstable region. Figure 5 gives a set of such curves in which voltage is plotted against scale reading. These curves were obtained by using different stabilizing weights, other conditions remaining unchanged. The sensitivity is seen to be low for curve $a$,

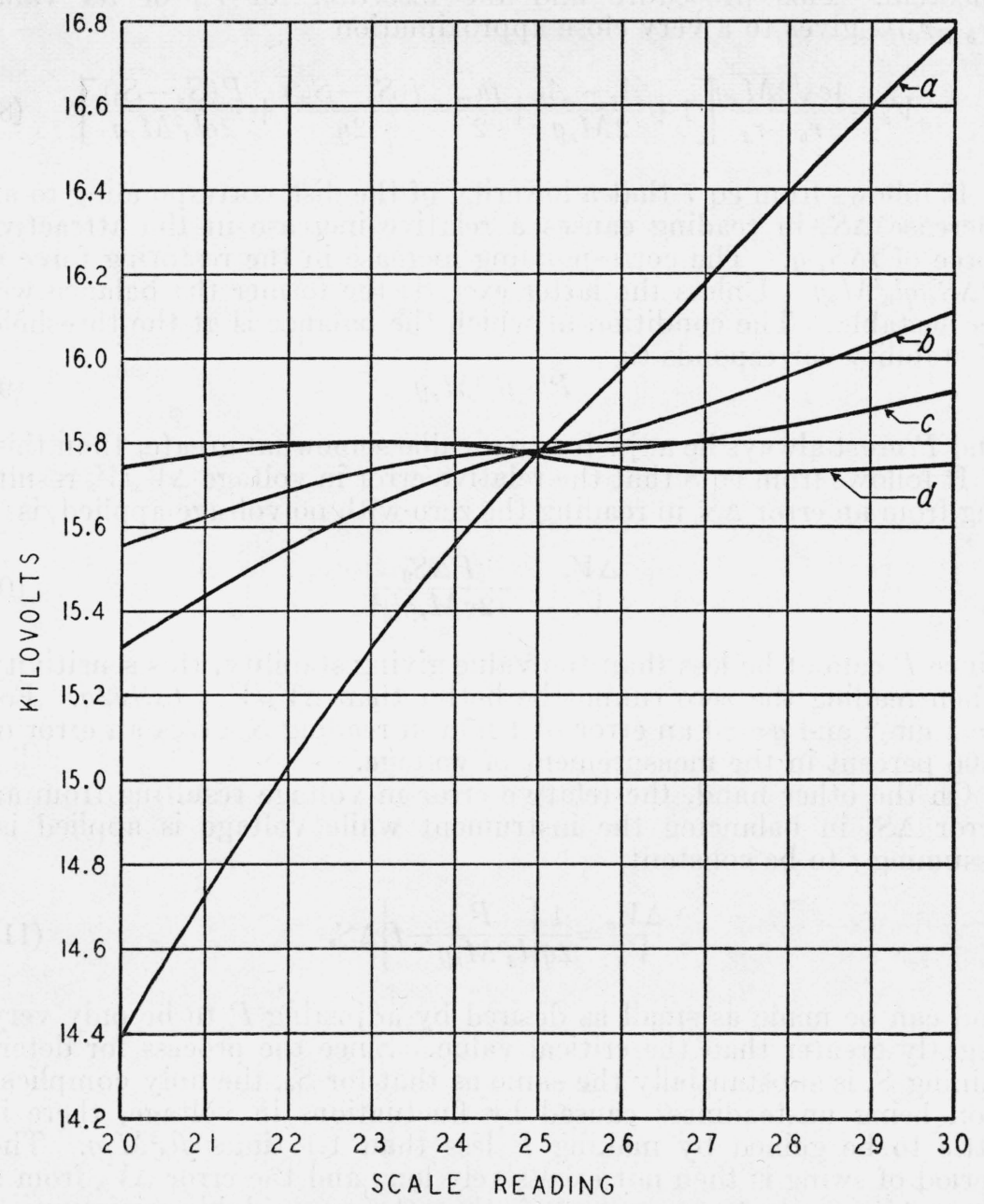

FiguRe 5.-Scale law of electrometer for various stabilities.

Disk and guard ring are coplanar at scale reading of 25. Curve $a$ taken with excessive stabilizing weight so that $\gamma / M_{F}=0.0248$; curve $b$ with normal stability $\gamma / M_{F}=0.0148$; curve $c$ with low stability $\gamma / M_{F}=0.0121$; curve $d$ with $\gamma / M_{F}=0.0098$, so that the movement is unstable between the 23- and the 27-cm positions but stable for greater displacements. Separation $=6 \mathrm{~cm}$, disk radius $=5 \mathrm{~cm}, M_{F}=250 \mathrm{mg}$.

to become higher for $b$ and $c$, and to be still high for $d$ but with an unstable range near the coplanar position. In this unstable range an increase in reading corresponds to a decrease in voltage. It may be noted that the existence of such a region in which the instrument is unstable at small deflections but stable at larger ones, can be predicted from the purely theoretical treatment of Snow. (See eq 50, section IX-3, p. 310.) 


\section{FORMULATION OF THE WORKING EQUATION}

In addition to the factors considered in the derivation of eq 8 above, a number of other corrections must be introduced to obtain the final working equation which gives the applied voltage in terms of the dimensions, scale readings and auxiliary measured quantities.

If the guard hoops are not maintained at exactly the proper relative potentials, a correction for the departure of their potentials from the ideal distribution must be applied. As shown in section IX-1, this correction can be put in the form $\sum_{k=1}^{k=\infty} C_{k} G_{k}$, where $G_{k}$ is a coefficient of the Fourier series which describes the departure of the actual potential distribution at the guard hoops from the ideal linear condition, and each $C_{k}$ is a numerical coefficient which can be calculated from the dimensions of the electrometer.

As already indicated, any lack of flatness of the disk requires both the addition of $h_{d}$ as a correction to the separation $c$ and the application of the empirically determined correction $\phi_{1}\left(h_{d} / r_{d}\right)$ to the voltage.

At extremely wide separations the distortion of the electric field in the spaces between the guard hoops may produce an appreciable effect. The experimentally determined correction for this is represented by the term $\phi_{2}\left(s / r_{h}\right)$ in eq 12 .

The dielectric constant $\epsilon$, of the atmosphere in which the electric field exists is not exactly unity and the voltage required to produce a given force is less on this account by the factor $1 / \sqrt{\epsilon}$, or approximately $1-(\epsilon-1) / 2$. For dry air at $0^{\circ} \mathrm{C}$ and $760-\mathrm{mm} \mathrm{Hg}$ pressure the dielectric constant is 1.000585 . The dielectric constant of moist air, ${ }^{9}$ however, is appreciably larger than that of dry air at the same total pressure and temperature, and for moist air we have used a correction calculated on the basis of a simple mixture rule. The total correction for dielectric constant in the present work was usually about 0.031 percent.

To express the voltage indicated by the electrometer in absolute electromagnetic volts, $V_{M}$, the value in electrostatic units $V_{s}$ must be multiplied by $v$, the ratio of the electrostatic unit to the electromagnetic unit. At present the factor 299.805 seems to be the best value. It results from the work of Rosa and Dorsey, combined with recent determinations of the ratio of the international and the absolute ohms.

By combining all of the various correction factors the following working equation of the instrument for small deflections results:

$$
\begin{aligned}
& V_{M}=\frac{4 v\left(c+h_{d}\right) \sqrt{2 M_{p} g}}{\left(r_{b}+r_{d}\right)}\left[1+\frac{A_{v}-A_{o}}{2 M_{P} g}+\frac{\gamma}{M_{r}}\left(S_{0}-S_{0}\right)\right. \\
& \left.\frac{f\left(S_{v}-S_{m}\right)}{2 q}+\frac{f h_{m}}{2}-\sum_{k=1}^{k=\infty} C_{k} G_{k}-\frac{\epsilon-1}{2}+\phi_{1}\left(\frac{h_{d}}{r_{d}}\right)+\phi_{2}\left(\frac{s}{r_{h}}\right)\right]
\end{aligned}
$$

- Delcelier, Guinchant and Hirsch. Onde Electrique 5, 189 (1926). 
In the use of the instrument, zero readings are always taken just before and just after the readings with voltage applied, and the unavoidable assumption is made that the air force $A_{v}$ while voltage is applied is the same as the mean, $A_{o}$, of the air forces during the zero readings. Although the second term in the bracket is therefore always taken to be zero, it has been retained throughout the derivation of eq 12 as a reminder that air forces are perhaps the largest uncontrolled source of error in the use of the instrument.

\section{PRECISION AND ACCURACY}

The usual process for obtaining an estimate of the accuracy of an instrument, namely, the comparison of its indications with those of a standard instrument known to be more accurate, could not be applied because no other standard of greater accuracy is available in this range.

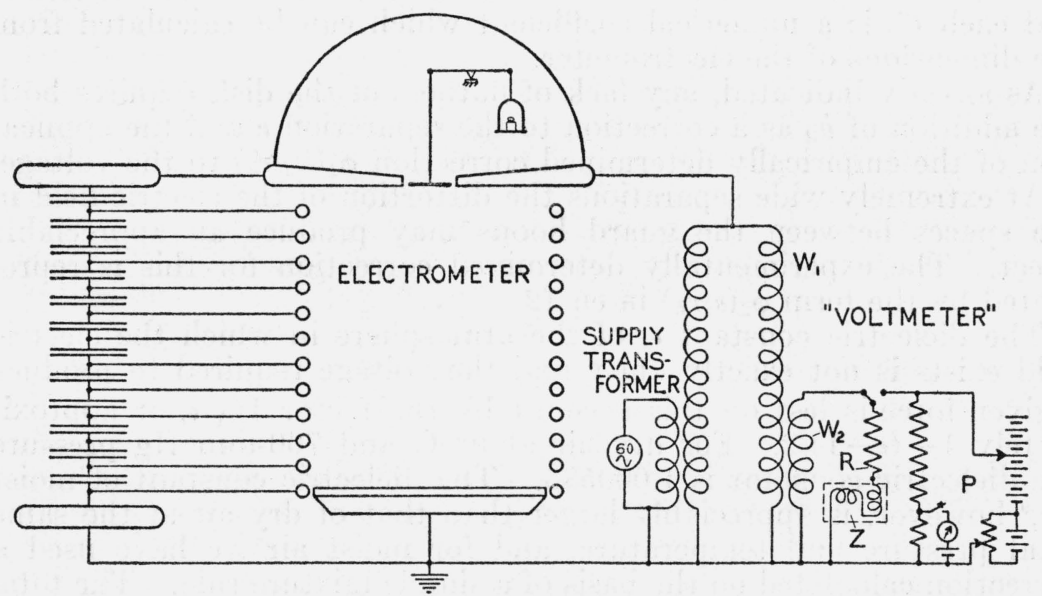
FIGURE 6.-Schematic diagram of connections , used in comparing electrometer with

The 230/100,000-volt step-up supply transformer is energized by a 60 -cycle 15 - $\mathrm{kva}$ alternator driven by a d-c motor supplied by storage batteries. The step-down transformer supplies the electrodynamic reflecting voltmeter $Z$ which has a series resistor $R$. The potentiometer circuit $P$ serves to calibrate the voltmeter.

The best comparison standard available consisted (as shown in fig. 6) of the combination of a step-down voltage transformer with a reflecting electrodynamic voltmeter $Z$ of the suppressed-zero type ${ }^{10}$ connected to its low-voltage winding $W_{2}$. The series resistor $R$ of this voltmeter was adjusted to keep the deflection approximately the same for all values of applied voltage. Because of the long scale distance and delicate suspension of this voltmeter, its sensitivity (16 $\mathrm{mm}$ for 0.1 percent change in voltage) is nearly 10 times that of the electrometer. For brevity, the combination of transformer and reflecting voltmeter will be referred to simply as the "voltmeter."

Immediately before and after each set of weighings, the reflecting voltmeter is checked against the electromotive force of a standard cell by the use of a potentiometer circuit $P$. Careful checks made recently, as well as at intervals during the past 15 years, have shown that the ratio of the voltage transformer for a given set of operating conditions remains constant to at least 0.02 percent.

\footnotetext{
10 F. K. Harris. A suppressed-zero electrodynamic voltmeter. BS J. Research 3, 445 (1929) RP105.
} 
In the course of this work a series of 64 independent comparisons of the electrometer with the "voltmeter" were made at various voltages from 10,000 to 100,000 . These comparisons were distributed over a variety of conditions which include: (a) The use of two disks of different radii; (b) five different separations between disk and lower plate; (c) six different combinations of series or parallel connection of the primary and the secondary windings of the stepdown transformer; (d) seven different values of magnetic flux density at which the transformer was operated; and (e) many different values of resistance in the calibrating potentiometer circuit. This work, using the technique finally developed, extended over a period of about 8 months. The scattering of these observations, as shown by the average difference taken without regard to sign, between the results given by the two independent methods, is 0.010 percent. It is, of course, impossible to separate this resultant scattering into the portions contributed respectively by the two methods of measurement, but it is certain that this figure constitutes an upper limit to the scattering which could have originated in either method. If any small secular changes had occurred in the ratio of the transformer during the progress of the work they would also have contributed to this observed scattering. The ratio of the transformer for certain connections as measured after the comparisons differed by 0.008 percent from the value observed before the comparisons. This fact suggests that some secular changes probably did occur.

An estimate of the precision of the individual readings is had from a study of curves such as $E$, figure 26 . Here differences between the two methods of measurement observed in random order but with voltage continuously applied at various scale readings, and hence with the disk at various heights above its guard ring, are plotted against scale reading. An examination of this and many similar curves shows that individual points seldom lie as much as 0.005 percent from a smooth curve.

If we consider the matter from the other point of view and deduce the probable accuracy of the whole by combining the accuracies of the parts, we find the situation to be as shown in table 1. Here the third column gives the observed or estimated values of the uncertainty of our measurements of the quantities listed in the first column. The observed values of uncertainty are deduced from data taken in the later part of the work, this uncertainty being expressed as the average, without regard to sign, of a number of differences between pairs of observations on supposedly the same quantity. In many cases the two observations of a pair were separated by a considerable lapse of time and hence the difference includes any secular drift during this time as well as the resultant errors of the two measurements themselves. In the three columns marked case 1 , case 2 , and case 3 , for three assumed sets of operating conditions, is given the uncertainty in the measurement of voltage which would result from an uncertainty in each quantity equal to the value given in the third column. At the bottom of these columns is given the resultant uncertainty to be expected from a random combination of the errors contributed by the several quantities listed.

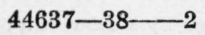


TABLE 1.-Probable uncertainty in component measurement and in voltage

\begin{tabular}{|c|c|c|c|c|c|}
\hline \multirow{2}{*}{ Quantity } & \multirow{2}{*}{ Symbol } & \multirow{2}{*}{$\begin{array}{l}\text { Uncertainty } \\
\text { observed or } \\
\text { estimated }\end{array}$} & \multicolumn{3}{|c|}{$\begin{array}{l}\text { Resulting percentage uncer- } \\
\text { tainty in voltage }\end{array}$} \\
\hline & & & Case 1 & Case 2 & Case 3 \\
\hline $\begin{array}{l}\text { Weight } \\
\text { Separation } \\
\text { Radius of disk and bushing } \\
\text { Zero setting (including air forees) } \\
\text { Voltage setting } \\
\text { Coplanar setting } \\
\text { Disk height } \\
\text { Disk concavity } \\
\text { Resistance arms of potential distribution bridge }\end{array}$ & $\begin{array}{c}M_{P} \\
c \\
r_{d}+r_{b} \\
S_{o} \\
S_{;} \\
S_{m} \\
h_{m} \\
h_{d} \\
R_{1} \text { or } R_{2}\end{array}$ & $\begin{array}{l}0.01 \mathrm{mg} \\
3.4 \mu \\
2.4 \mu \\
0.04 \mathrm{~mm} \\
0.04 \mathrm{~mm} \\
0.06 \mathrm{~mm} \\
0.8 \mu \\
2.2^{\mu} \\
0.01 \mathrm{ohm}\end{array}$ & $\begin{array}{l}0.000_{2} \\
.005_{8} \\
.001_{5} \\
.003_{6} \\
.000_{3} \\
.005_{0} \\
.005_{2} \\
.002_{0}\end{array}$ & $\begin{array}{l}0.000_{2} \\
.002_{8} \\
.001_{5} \\
.010_{0} \\
.008_{0} \\
.005_{0} \\
.005_{2} \\
.003_{0}\end{array}$ & $\begin{array}{l}0.0008 \\
.0007 \\
.001_{8} \\
.005_{7} \\
.002_{2} \\
.005_{0} \\
.005_{0} \\
.004_{0} \\
.000_{0}\end{array}$ \\
\hline $\begin{array}{l}\text { Resultant uncertainty (square root of sum of } \\
\text { squares) }\end{array}$ & & & & .014 & .010 \\
\hline
\end{tabular}

Case 1 is for $c=6 \mathrm{~cm}, V=30,000$ volts, $M_{F}=2,200 \mathrm{mg}$, stability coefficient $\gamma=1.1$ times its value at the threshold of instability.

Case 2 is for $c=12 \mathrm{~cm}, V=60,000$ volts, $M_{F}=2,200 \mathrm{mg}$, stability coefficient $\gamma=3.5$ times threshold value.

Case 3 is for $c=48 \mathrm{~cm}, V=120,000$ volts, $M_{F}=550 \mathrm{mg}$, stability coefficient $\gamma=1.7$ times threshold value.

In the case of the quantity $h_{m}$ the value given under the column headed "uncertainty" is the difference in the two values of $h_{m}$ observed at the beginning and end of the set of weighings on the same day, the value, $0.8 \mu$, being the mean for 20 days. This measurement involves both the act of setting the vernier image to the scale reading $S_{m}$ and the measurement of the resulting $h_{m}$ with the coplanarity microscopes. The item $0.06 \mathrm{~mm}$ for the uncertainty in $S_{m}$ is the same value expressed as a distance on the scale. Either uncertainty taken alone covers the entire operation and the two should therefore not be combined.

The value $0.04 \mathrm{~mm}$ for $S_{o}$ is the mean of 20 pairs of observations, the members of each pair being obtained just before and just after a series of weighings, the time interval being on the average, $1 / 2$ hour. As each "observation" is itself the mean of 30 readings, the true errors of reading have largely cancelled out and the result is rather an index of the changes in air force between beginning and end.

Similarly, the value for the uncertainty in $S_{v}$ is deduced from the average deviation from their mean, without regard to sign, of the $n$ individual readings (in most cases $n=10$ ) taken during a single weighing, by dividing this mean deviation by $(n-1)^{1 / 2}$. The value given, which is the mean of 10 such separate sets taken on different days, is largely a measure of variations in air currents combined with uncertainties resulting from unsteadiness in the applied voltage, ${ }^{11}$ rather than a simple reading error.

An air force of $0.1 \mathrm{mg}$, which would produce an error of 0.01 percent in voltage under typical conditions, could be produced by a convection current having a velocity of about $1 \mathrm{~cm} / \mathrm{sec}$. It is therefore evident that such forces will materially reduce the precision otherwise attainable by a single reading with this instrument. On the other hand, the natural fluctuations in such air currents should cause their effects

\footnotetext{
11 In the present work a 15-kva alternator of the revolving-field type was driven by a 230-volt d-c motor supplied by a 50-ampere storage battery, while the alternator field was supplied by a second battery. In spite of this arrangement and of the great pains taken to keep the bearings and brushes in prime condition, irregular fluctuations of the order of 0.01 percent were nearly always present.
} 
to largely cancel each other when the mean of a fairly large number of readings is taken. The small scattering of the zero readings noted in table 1 is evidence for this.

It appears from an inspection of table 1 that the uncertainty in the measurement of weight, potential distribution, disk radius, separation (at large separations) and deflection setting (when working with a small margin of stability), could produce only negligible errors. On the other hand, errors of 0.005 percent may well be introduced by uncertainty in disk flatness, disk height, zero setting, separation (at small separations), and in deflection setting at high stability. If a random combination of their effects be assumed, the over-all uncertainty in any one complete determination of voltage may therefore be expected to have the value marked "resultant uncertainty" in table 1.

\section{EXPERIMENTAL TESTS}

During the later stages of the development of the instrument a rather elaborate program of experimental work was carried out with it to determine its possibilities and limitations. Because of the small size of the high-voltage laboratory of the National Bureau of Standards, and the voltage rating of the available transformers, this experimental work was limited to 100,000 volts. However, it included measurements at potential gradients exceeding those which will normally be present in work at 275,000 volts, and also measurements at the full separation needed for this latter voltage.

Because of its intended use as an absolute instrument, independent of other methods of high-voltage measurement, it was essential that all necessary tests should be applied to insure that the construction and the functioning of the electrometer were in sufficiently close agreement with the ideal conditions for which its theoretical equation was derived. For this purpose various rather drastic departures from the ideal conditions were intentionally introduced and the corresponding effects determined by comparing the indications of the electrometer with those of the "voltmeter" described above, which was of course left unaffected ${ }^{12}$ during each experiment.

In order that no disturbance should occur, other than the change in condition intentionally introduced in each experiment, the mass $M_{F}$ was left unchanged, and any resulting change in the voltage necessary to restore the balance of the instrument was derived from the readings of the "voltmeter."

Whenever feasible, runs with such changed operating conditions were immediately preceded and followed by check runs under normal conditions. This procedure tends to eliminate any secular drift in the calibration of either instrument. The constancy of the transformer allows us to place nearly as much confidence in observed differences for changed conditions on different days as when the runs under normal and changed conditions were made on the same day. For the purposes of this paper the absolute calibration of the transformer-electrodynamometer combination need not be considered. The results given below are expressed as a "relative difference" which is the percentage by which the indication of the electrometer exceeded that of the "voltmeter," both being reduced to the common basis of absolute

${ }_{12}$ Unfortunately, during the temperature runs, the transformer was necessarily exposed to the changes in room temperature. 
electromagnetic volts. Practically all the measurements were made at a frequency of 60 cycles per second.

It is to be noted that a number of the measurements reported here were made early in the work before certain refinements of technique had been developed. The scattering in the early results should therefore not be taken as a measure of the precision attainable by the later procedures.

\section{ELECTRICAL DISCHARGES}

In addition to the many ways in which an electrometer may fail to satisfy the purely geometrical requirements of its theory, there is the further possibility, in an instrument operating at high voltage, that some brush discharge or other partial breakdown of the dielectric may produce abnormal conditions. The possibility of such a discharge appears evident when one considers that, if the disk has a sharp square edge, the electric gradient at that edge is theoretically infinite even if the disk is withdrawn into the aperture in the guard. Should a discharge be present there may well be mechanical forces set up by the resulting "electric wind," and in addition the distortion of the electric field by the resulting ionization and space charges may possibly be appreciable.

A considerable number of tests were therefore planned with a view to produce or to modify such a discharge, or to imitate to an exaggerated extent its effects so that the result might be observed. These tests were made with spacings of $12 \mathrm{~cm}$ or less so that the guard hoops and their potential divider were not needed. This permitted the use of average gradients running up to 5,300 volts (effective) per $\mathrm{cm}$ at which sparks would occasionally pass between the edge of the upper plate and the rather sharp edge of the lower plate. In no case did a spark pass to the disk itself. The results of this group of tests are summarized in table 2. The seventh column shows the change in the required applied voltage resulting from the change in conditions.

In set $A$, with the disk tilted about a diameter through an angle of 6 minutes, the edge protrudes at one side by $0.09 \mathrm{~mm}$, this being nearly the extreme range measurable with the coplanarity microscopes. The force from any electric wind then present would be expected to vary materially with both the tilt and the applied voltage and thus reveal itself. The changes shown in the seventh column of table 2 are less than the precision at the time these experiments were made.

In set $B$ the power supply was obtained from a 60 -cycle and a 180 -cycle generator connected in series, the latter delivering about 30 percent as much voltage as the former. By reversing or by opening the field circuit of the latter, the wave form could be markedly varied, although the effective value was kept adjusted to the constant value required to balance the mass $M_{F}$. To eliminate any effect of a change in transformer ratio with wave form, the step-down transformer was omitted and the electrodynamometer was used in series with a shielded resistor of about $400,000 \mathrm{ohms}$. It might be expected that with the peaked wave form, by reason of the greater crest value of voltage, there would be more discharge than under the other two conditions. It is seen from table 2 that no change was detected though the voltage was almost sufficient to cause sparkover. 
TABLE 2.-Effects of various abnormal conditions



sDischarge if present should depend upon the magnitude of this quantity, "gradient" = effective voltage Xcrest factor $\div$ plate separation.

bThis column gives the voltage as indicated by the "voltmeter."

- At the time of these observations the precision of measurement was no better than \pm 0.04 percent.

dFigure 7 shows distribution of cotton lint during this experiment.

The tests in set $C$ were made by rounding off the lower edge, originally rectangular, of one disk to a radius of about $0.02 \mathrm{~mm}$. Line $X X$, figure 4 , p. 260 , marks the center of the radius of rounding. This rounding should reduce the gradient, which is theoretically infinite for a rectangular corner, to a very moderate value. The slight difference observed in the readings may perhaps be the result of some general distortion of the disk produced by the rounding operation, in spite of the fineness of the stone and the gentleness of the grinding force used.

In normal operation the disk, guard, and lower plate were frequently wiped with soft leather soaked in benzol and were therefore reasonably free from dust particles. To see whether such particles as did elude our vigilance could make an appreciable error, a number of cotton fibers were caused to cling to the attracted disk. This was done by placing cotton lint on the lower plate and connecting an a-c and a d-c source in series between the plates. Figure 7 shows the resulting condition which obtained during this experiment. In spite of this very drastic treatment no consistent change in the performance of the electrometer appears in set $D$. 
The tests of set $E$, in which either a source of direct voltage or a condenser was connected between the disk and its guard ring were intended to roughly simulate the effect of any possible electrical double layer on the surface such as might result from an electrical discharge or other cause. As shown in table 2, no effect was observed at 1.5 volts. At 4.5 volts the disk pulled over to the guard ring and adhered. Hence it may be assumed that there can be no rectifying action present which might cause an accumulation of charge.

\section{TILT OF DISK AND OF LOWER PLATE}

Departure from the ideal condition of parallelism between the disk and the upper and the lower plate was also studied. The data given in section $A$ of table 2 for cases in which the disk is considerably tilted with respect to the guard ring and the lower plate show that no large error need be expected from the unavoidable slight tilt of the disk. Section $F$ of table 2 gives similar data obtained when the lower plate was intentionally tilted at an angle of about $9^{\prime}$. These data indicate that the effect of tilt in normal operation must be very small, for the lower plate is always adjusted to have a tilt less than $1^{\prime}$.

\section{ECCENTRICITY OF DISK IN APERTURE}

Theoretically, one would expect that small horizontal displacements of the disk from its central position in the opening in the guard ring would have little or no effect on the attractive force for a given voltage, because such displacements do not alter the area of the gap. To test this point experimentally, weighings were made with the $16-\mathrm{cm}$ disk and $12-\mathrm{cm}$ separation. The results are given as set $G$ of table 2. No guard hoops were used with this short separation. The greatest displacement used $(0.025 \mathrm{~cm})$ was such as to make the disk very nearly touch the guard ring at one side when no voltage was applied. It is natural to assume that the electrostatic forces tend to center the disk, so that the actual eccentricity when measuring an applied voltage may have been less than the values observed with no voltage applied. It is evident from the very small mean difference given in the seventh column of table 2 that the effect of an initial eccentricity is negligible.

\section{WIDTH OF GAP}

Of the purely geometrical factors to be considered the most obvious is the gap which must be left between the disk and its guard ring, if the former is to move without friction. The early work of Maxwell, and more particularly the recent paper of Snow ${ }^{13}$ indicate that the effect of this gap, of width $a$, can be properly allowed for, provided the disk and ring are coplanar, by adding half the radial width of the gap to the radius $r_{d}$ of the disk itself. From the symmetry of the geometrical system treated by Snow, it is evident that his result can give no information as to terms of higher order in $a / r_{d}$, which might be appreciable in the case of disks of small radius and large gap. Such effects were therefore sought experimentally by a series of measurements in which a disk initially $10 \mathrm{~cm}$ in diameter having a radial clearance of $0.0055 \mathrm{~cm}$ was reduced in diameter by successive

${ }_{13}$ Chester Snow. Effect of clearance and displacement of attracted disk, and also of a certain arrangement of conducting hoops upon the constant of an electrometer. BS J. Research 1, 513 (1928). RP17. 


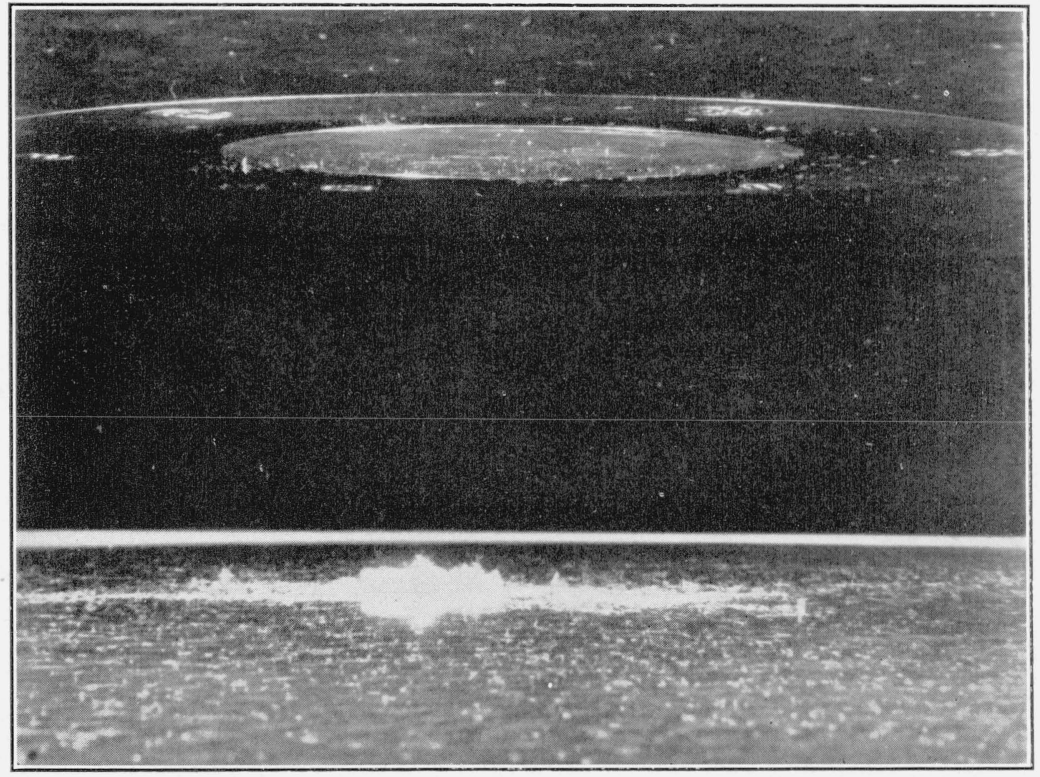

FIGURE 7.-Condition of disk and guard ring after lint had been applied for measurements given in table 2 , part $D$.

The disk, guard ring, and gage-rod holes (all much foreshortened) are visible at the top and the pile of lint on the lower plate below. 




FiguRE 11. Optical interference pattern formed by active surface of 10-cm bushing insert and a glass flat. 
small amounts until the radial clearance was $0.26 \mathrm{~cm}$. The results, shown in figure 8 , shows that the voltage as deduced by eq 12 , using the mean of the radius of the disk and that of the aperture, was not affected by more than 0.05 percent up to a gap of $0.26 \mathrm{~cm}$ or nearly 1.7 times the thickness of the disk. This somewhat remarkable result is consistent with a rough theoretical estimate based on Snow's work, which shows an astonishingly small penetration of lines of force in to the gap even when it is wide. At the larger gaps the air damping of the moving system was greatly reduced and the operation of the instrument became very inconvenient. With a wide gap it is also necessary to slide the coplanarity microscopes through a greater range to sight on the disk and on the guard, and the possibility of error from irregularities in their supporting grooves is increased. For gaps of less than $0.005 \mathrm{~cm}$ the damping is excessive, mechanical center-

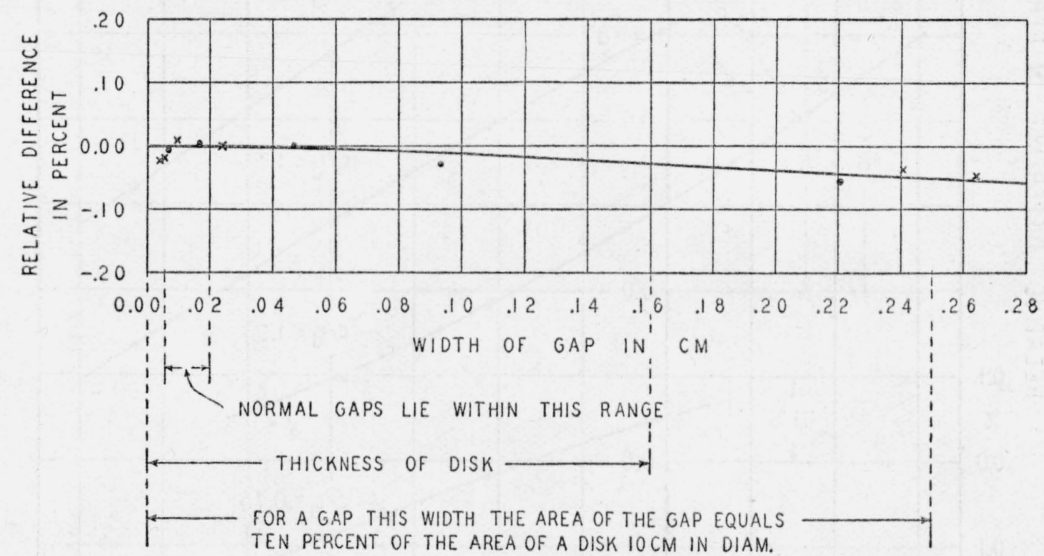

Figure 8.-Effect of width of gap.

Ordinates are the relative difference between the indication of the "voltmeter" and that of the electrometer as computed by adding half the width of the gap to the radius of the disk as required by eq 12 . Dots correspond to a series of measurements in which a disk initially $10 \mathrm{~cm}$ in diameter was turned down in successive stages. Crosses correspond to a series in which the aperture in the guard ring was enlarged.

ing adjustment of the disk to provide clearance becomes tedious, and dust particles that lodge in the gap produce frictional trouble more readily. A gap in the range between 0.006 and $0.02 \mathrm{~cm}$ gives satisfactory performance. From figure 8 the effect of terms of higher order in $a / r_{d}$ for gaps within this range is seen to be negligibly small.

\section{FLATNESS OF DISK AND OF GUARD RING}

Early in the work discrepancies between results obtained with different disks indicated that some previously unsuspected source of constant error was present. A considerable number of possible sources of error were investigated and the discrepancies were finally traced to the fact that the disks first used were not sufficiently plane. Figure 9 shows results obtained on a number of disks of varying degrees of flatness. Here the ordinates are the relative differences in the indications of the electrometer and the "voltmeter," while the abscissas are the values of $h_{d} / r_{d}$, i. e., the ratio of the mean height of the lower surface of the disk above its rim, to the radius of the disk. (If the curvature is constant this ratio is proportional to the ratio of the 
radius of the disk to that of the spherical surface of which the lower surface of the disk forms a segment.) Positive abscissas correspond to concave disks. The radii of curvature $R_{c}$ (in meters) of the spherical surfaces of several of the $10-\mathrm{cm}$ disks are indicated along the axis at the corresponding values of $h_{d} / r_{d}$.

The shape of the active surface of most of the disks used approximated more or less closely to that of a segment of a sphere. Others,



Figure 9.-Residual effects of lack of flatness of disk.

Abscissas are effective curvature expressed by the ratio of the mean height, $h_{d}$, of lower surface of the disk above the mean level of its rim, to its radius $r_{d}$. A plane disk has $h_{d} / r_{d}=0$. Positive values correspond to concave disks. The values indicated by $R_{c}$ are the radii in meters of a spherical surface of which the lower surface of a disk at that abscissa wculd be a segment. The ordinates are the relative differences between the indications of the voltmeter and those of the electrometer after allowance has been made for $h_{d}$ by using $c+h_{d}$ as the separation. Each curve corresponds to a different value of $c / r_{d}$. To avoid confusion each curve is drawn to a different axis of abscissas. Points $a, a^{\prime}$, and $a^{\prime \prime}$ show data obtained with an initially nearly spherical concave 10-cm disk before and after the sur face had been reworked; $b$ and $b^{\prime}$ show results before and after reworking a 10-cm convex disk. $c^{\prime}$ and $c^{\prime \prime}, d$ and $d^{\prime}$ show data obtained on built-up disks which were not so nearly parts of spherical surfaces. Distance $A$ to point $b_{o}$ shows total effect of $h_{d}$ in this case, no allowance being made for lack of flatness. Distance $B$ to point $b$ shows the residual effect after allowing for the direct effect of $h_{d}$ on spacing.

such as those corresponding to points marked $a^{\prime}, b^{\prime}, c^{\prime}$, and $d^{\prime}$, were of somewhat different shape. The nearly linear relation between relative difference and $h_{d} / r_{d}$ is perhaps fortuitous and may not apply to disks which depart markedly from the spherical shape. It is felt, however, that for the disks here used, the coefficients obtained from the slope of the plotted curves should suffice for computing the correction 
to be applied to the nearly flat disks used in the best measurements. Irregularities of smaller scale than the general curvature and not near the edge would be expected merely to shift the charge from one part of the disk to another without much effect on the total force.

The relative importance of a given distortion $h_{d}$ of the disk in its influence on the effective spacing and on the change in charge distribution is indicated by points $b_{o}$ and $b$. The former was obtained by entirely ignoring $h_{d}$ in the computation of the voltage as indicated by the electrometer, using eq 12 , and this result differs from the ideal

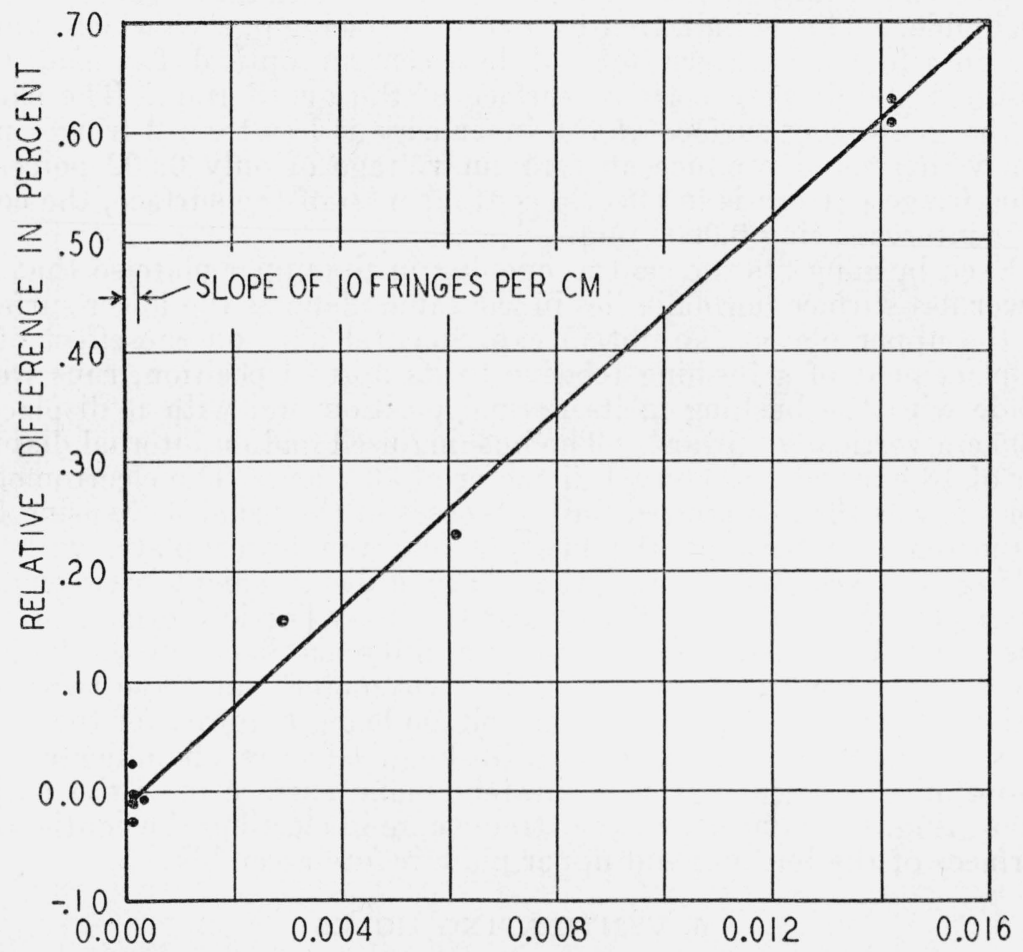

SLOPE OF BUSHING NEAR INNER EDGE (CONVEX)

Figure 10.-Effect of lack of flatness of guard ring.

Abscissas are the slope of the surface at the edge of the aperture. Ordinates are relative difference between electrometer and "voltmeter."

result for a flat disk by the amount $A$. The same data when using $c+h_{d}$ for the spacing but still ignoring the correction term $\phi_{1}\left(h_{d} / r_{d}\right)$, yield point $b$, which still differs by the amount $B$ from the ideal.

This remaining difference $B$ from the value corresponding to a plane disk must be the result of some additional effect of the somewhat irregular convexity of the disk used and comes presumably from the tendency of the electric charge to crowd toward a convex and retreat from a concave part of the surface. It is this contribution which is represented by $\phi_{1}\left(h_{d} / r_{d}\right)$ in eq 12 .

The slope of the curves in figure 9 shows that this additional effect is less at close separa tions $\left(c / r_{d}=0.75\right)$ and increases with the separation. 
For separations for which $c / r_{d}$ exceeds 1.5 the effect becomes nearly independent of separation.

A corresponding effect is to be expected if the surface of the guard ring near the disk is conical. Figure 10 shows the results of a series of measurements in which the inner portion of the guard ring extending for a distance of $5 \mathrm{~cm}$ from the gap was initially made conical, and then made flatter in successive stages. A 10-cm disk was used in these experiments. The error in voltage is seen to be proportional to the slope of the conical surface. In the guard rings finally used this slope could readily be made small enough to make this source of error negligible. This is indicated by figure 11 (facing p. 273) which shows the interference pattern formed between an optical flat and the polished stainless-steel active surface of the guard ring. The slope of the guard-ring surface at the inner edge is less than 2 fringes per $\mathrm{cm}$, which could produce an error in voltage of only 0.002 percent. This fringe pattern is in effect a contour map of the surface, the contour interval being $0.0003 \mathrm{~mm}$.

Each bushing fits the central opening in the upper plate so that its lower flat surface normally lies in the same plane as the lower surface of the upper plate. To obtain experimental data on the effect of a displacement of a bushing relative to its normal position, runs were made with the bushing in its normal position and with it displaced $0.05 \mathrm{~cm}$ vertically upward. The bushing used had an internal diameter of $16 \mathrm{~cm}$ and an external diameter of $30.2 \mathrm{~cm}$. The electrometer voltage was then computed for both cases in the usual way, using for $c$ the distance between the bushing and the lower plate, without taking into account the resulting relative displacement between the outer portions of the guard ring and the inner portion formed by the bushing. The change in the relative difference between the indications of the electrometer and of the "voltmeter" for these two conditions was 0.38 percent, a higher voltage being required for the same force in the case of the displaced bushing. The result indicates the importance of securing an accurately plane surface over the entire guard ring, which necessitates extreme care in cleaning the contiguous surfaces of the bushing and upper plate before assembly.

\section{VENTILATING HOLES}

It was originally planned to close the six gaging holes $(20 \mathrm{~mm}$ in diameter) in the guard ring by means of tight-fitting solid plugs whose lower ends were flush with the lower plane surface of the guard ring. Experience showed, however, that the closing of these holes in this manner increased the difficulties from air currents and made the damping excessive. Consequently, perforated plugs (9) figure 27 (see also fig. 16) are used which permit a rapid equalization of air pressure on the two sides of the disk. Because the centers of these plugs are only $13 \mathrm{~cm}$ from the center of the attracted disk, and the holes $(4 \mathrm{~mm}$ in diameter) in the bottom of each plug break the continuity of the plane surface of the guard ring, it seemed desirable to obtain a satisfactory experimental check before considering their effect to be negligible. Such a check was made by removing three alternate plugs, stopping the holes in the upper side of the guard ring with corks, and then taking observations in the usual way. As customarily used with the plugs in place, the total area of the ventilating openings is 
$404 \mathrm{~mm}^{2}$. With the three plugs removed this total area is increased to $1,106 \mathrm{~mm}^{2}$. The mean of readings taken before removing and after replacing the plugs gives a value only 0.005 percent lower than the mean of readings taken with the plugs removed. This shows that the effect of the ventilating holes can be neglected.

\section{TEMPERATURE}

The primary effects of an increase in temperature on the indications of the electrometer arise from: (1) The expansion of the disk and of the aperture in the guard ring, both of which increase the effective area, and (2) vertical expansion of the silica pillars, of the invar rods supporting the lower plate, and of the lower plate itself, the net effect of which is a decrease in the separation. There is, therefore, a net increase in the force of attraction and a decrease in the voltage required for a given force. The temperature coefficient of voltage for a given force as calculated is $-89 \times 10^{-6} \mathrm{per}^{\circ} \mathrm{C}$ at a separation of 6 $\mathrm{cm}$ and $-24 \times 10^{-6} \mathrm{per}^{\circ} \mathrm{C}$ at $48 \mathrm{~cm}$. The construction of the instrument is such as to minimize any other temperature effects, but as an experimental check to detect any large unsuspected temperature effects, observations were made in June 1933 at temperatures ranging from 25 to $34^{\circ} \mathrm{C}$ with a separation of $6 \mathrm{~cm}$. These observations indicated that, after the calculated corrections mentioned above had been applied, there remained no residual temperature effect greater than the precision of the determinations. Because of the small range of temperature (only $9^{\circ} \mathrm{C}$ ) and the fact that the voltage transformer as well as the electrometer was necessarily subjected to the change in temperature, the precision of these determinations was probably not better than $30 \times 10^{-6}$ per ${ }^{\circ} \mathrm{C}$ in temperature coefficient of voltage.

\section{GUARD-HOOP POTENTIALS}

As the guard hoops constitute the most novel feature of the instrument, a number of experimental tests will now be described which were made to determine their applicability and limitations.

The derivation of an equation giving the voltage required to produce a given force on the disk, in terms of the dimensions of the instrument and the relative potentials of all points on an imaginary cylindrical surface tangent internally to the set of hoops, is given in section IX-1. This deduction is based on the assumption that the instrument and the potential distribution are axially symmetrical. It is convenient to express the potential $V_{R}(y)$ of a point on this cylindrical surface at a height $y$ above the lower plate by the equation

$$
V_{R}(y)=V_{s}\left[\frac{y}{b}+\sum_{k=1}^{k=\infty} G_{k} \sin \frac{k \pi y}{b}\right]
$$

Herein $V_{s}$ is the total applied voltage, $b$ the separation between disk and lower plate, $k$ is an integer, and the Fourier series in the second term describes the departure of the potential distribution from the ideal uniform gradient. Conversely, each coefficient $G_{k}$ is connected with the potential distribution by the relation

$$
G_{k}=\frac{2}{b} \int_{0}^{b}\left(\frac{V_{R}(y)}{V_{s}}-\frac{y}{b}\right) \sin \frac{k \pi y}{b} d y
$$


It is proved in section IX-1 that to a first approximation the potential difference $V_{s}$ required to produce a force $F$ on a central disk of radius $r_{d}$ is

$$
V_{s}=\frac{2 b \sqrt{2 F}}{r_{d}}\left[1-\sum_{k=1}^{k=\infty} C_{k} G_{k}\right] \text { approximately, }
$$

where the coefficients $C_{k}$ are given by

$$
C_{k}=(-1)^{k} \pi k \frac{2 J_{1}\left(\frac{i k \pi r_{d}}{b}\right)}{\frac{i k \pi r_{d}}{b} \times J_{0}\left(\frac{i k \pi R}{b}\right)}
$$

$J_{0}$ and $J_{1}$ being Bessel's functions with parameters 0 and 1 , respectively.

Values of the coefficients $C_{k}$ may readily be computed from the dimensions of the electrometer and are given in table 3 for an instrument of the present proportions.

TABLE 3.-Typical values of the coefficients, $C_{\boldsymbol{k}}$

\begin{tabular}{|c|c|c|c|c|c|}
\hline$C_{k}$ & $\begin{array}{l}b=6 \mathrm{~cm} \\
n=2\end{array}$ & $\begin{array}{l}b=12 \mathrm{~cm} \\
n=5\end{array}$ & $\begin{array}{l}b=24 \mathrm{~cm} \\
n=11\end{array}$ & $\begin{aligned} b & =48 \mathrm{~cm} \\
n & =23\end{aligned}$ & $\begin{aligned} b & =110 \mathrm{~cm} \\
n & =54\end{aligned}$ \\
\hline $\begin{array}{l}C_{1} \\
C_{3} \\
C_{3} \\
C_{4}\end{array}$ & $\begin{array}{l}-4.1 \times 10^{-0} \\
+4.8 \times 10^{-18} \\
-6.5 \times 10^{-27} \\
\end{array}$ & $\begin{array}{l}-2.0 \times 10^{-4} \\
+8.4 \times 10^{-0} \\
-8.1 \times 10^{-15} \\
-\end{array}$ & $\begin{array}{r}-4.7 \times 10^{-2} \\
+4.1 \times 10^{-6} \\
-3.0 \times 10^{-6} \\
\hdashline\end{array}$ & \begin{tabular}{l}
-0.621 \\
+.090 \\
$-8.9 \times 10^{-3}$ \\
\hdashline
\end{tabular} & $\begin{array}{r}-2.094 \\
+1.678 \\
-0.847 \\
+.358 \\
-.012\end{array}$ \\
\hline$C_{25}$ & & & $-\cdots$ & $\begin{array}{l}-3.9 \times 10^{-27} \\
+7.4 \times 10^{-53}\end{array}$ & - \\
\hline$C_{50}$ & & & & $\mid-1,1$ & $\begin{array}{l}+4.2 \times 10^{-28} \\
+1.7 \times 10^{-52}\end{array}$ \\
\hline
\end{tabular}

[ $r_{d}=8 \mathrm{~cm}, R=47.25 \mathrm{~cm}$.]

Here $n$ is the number of intermediate hoops which would be present when using the normal hoop spacing of $2 \mathrm{~cm}$. It will be seen that the values of $C_{k}$ are very small at small separations, which means that the hoops are then unnecessary. At larger separations the values are quite appreciable but decrease rather rapidly with increase in $k$, so that for values of $k$ comparable with the number of hoops, $C_{k}$ is vanishingly small.

Unfortunately, the correct values of $G_{k}$ as defined by eq 14 cannot be determined because the potential $V_{R}(y)$ is not known at all points. However, for the particular values of $y$ at which the hoops touch the imaginary cylindrical surface of radius $R$ the values of $V_{R}(y)$ are necessarily the same as the potentials of the hoops. The potential of any hoop is fixed by the capacitances of the potential divider units combined with the stray capacitances between the various hoops and from the hoops to ground and also to metal parts at high potential. The ratio of the potential of a hoop above that of the lower plate to the total potential difference $V_{s}$ is equal to the ratio of the effective capacitive reactance between the hoop and ground to the total capacitive reactance. This ratio is measured for each 
hoop, as described in detail in section VIII-6, page 299, by using an audio-frequency bridge. It is assumed that when the electrometer is used with a high 60-cycle voltage, this ratio of reactances is the same as when measured with about 380 volts at 1,000 cycles per second.

The points plotted in figure 12 show the potentials of the hoops in terms of the total applied difference $V_{s}$, as thus observed for the full separation, $110 \mathrm{~cm}$, when 54 intermediate hoops are used. The straight line $A$ corresponds to a uniform potential gradient. It will be noted that the hoops are rather systematically at a lower potential than the ideal, the maximum distortion amounting to about 3 percent of $V_{s}$. The scale of the figure is too small to show the minor

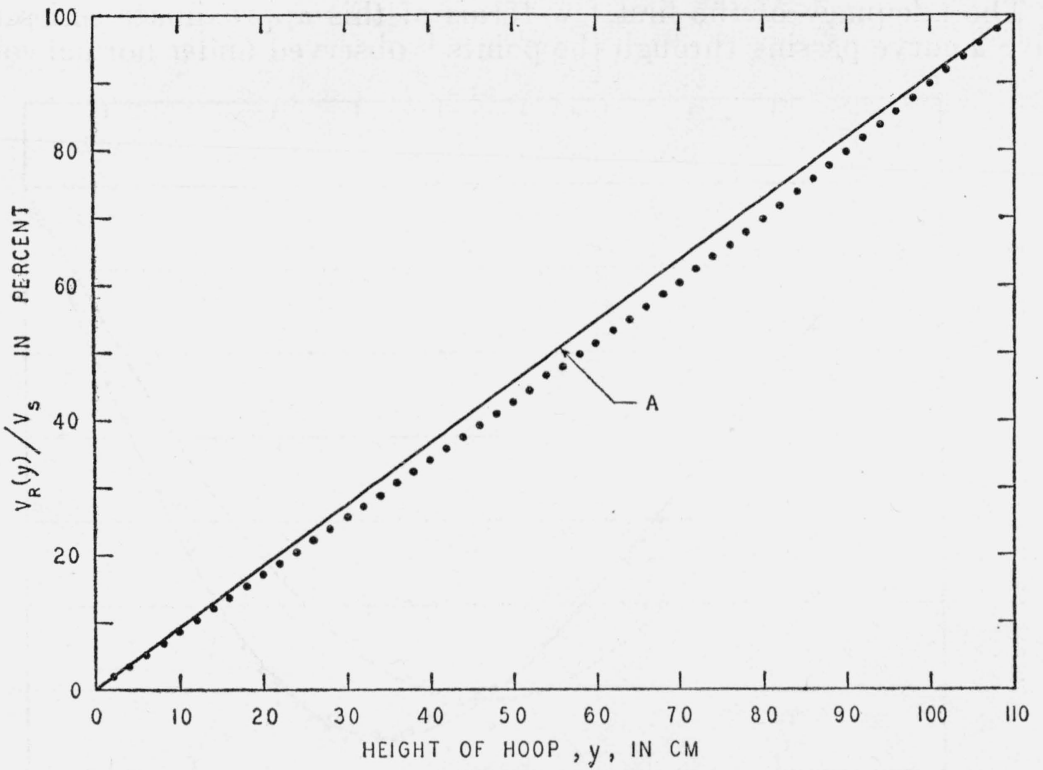

FigURE 12.-Typical potential distribution curve

The plotted points show the potentials of the hoops with respect to the total applied voltage as deduced from measurements made with the audio-frequency bridge. The line $A$ corresponds to the ideal uniform potential gradient. Abscissas are the heights of the hoops above the lower plate. Data were obtained with a separation of $110 \mathrm{~cm}$ and a hoop spacing of $2 \mathrm{~cm}$.

irregularities which result from the slight inequalities in capacitance of the various units of the potential divider.

Although the appearance of figure 12 suggests that the potential varies according to a smooth curve through the plotted points, it must be remembered that no information can be obtained by direct experiment about the values of potential at intermediate points. In fact, it is not only possible but for theoretical reasons certain, that the curve of potential versus height must contain superposed ripples of wave length equal to the hoop spacing. The origins and possible effects of such ripples are discussed in section IX-2, page 305 .

If the ripples are neglected, it is possible to compute, from the observations made with the audio-frequency bridge, a set of approximate Fourier coefficients, which we may denote by $G_{k}{ }^{\prime}$. The requirement that the curve defined by these $n$ coefficients shall pass through 
the $n$ observed points gives $n$ linear equations involving $G_{1}{ }^{\prime}, G_{2}{ }^{\prime}$. . . . $G_{n}{ }^{\prime}$. The solution of these equations gives for the $k$ th term

$$
G_{k}{ }^{\prime}=\frac{2}{n+1} \sum_{m=1}^{m=n}\left(\frac{V_{m}}{V_{s}}-\frac{m}{n+1}\right) \sin \frac{k m \pi}{n+1} k=1,2 \ldots n
$$

Table 4 gives values of $G_{k}{ }^{\prime}$ which are typical of normal operation at separations of 48 and $110 \mathrm{~cm}$, and shows the rapid decrease of $G_{k}{ }^{\prime}$ with increase in $k$. This decrease makes the terms of higher order in $\Sigma C_{k} G_{k}{ }^{\prime}$ negligible even at the wide separations where the rate of decrease in $C_{k}$ alone is slow.

The adequacy of the first few terms of this approximate series to give a curve passing through the points ${ }^{14}$ observed under normal con-

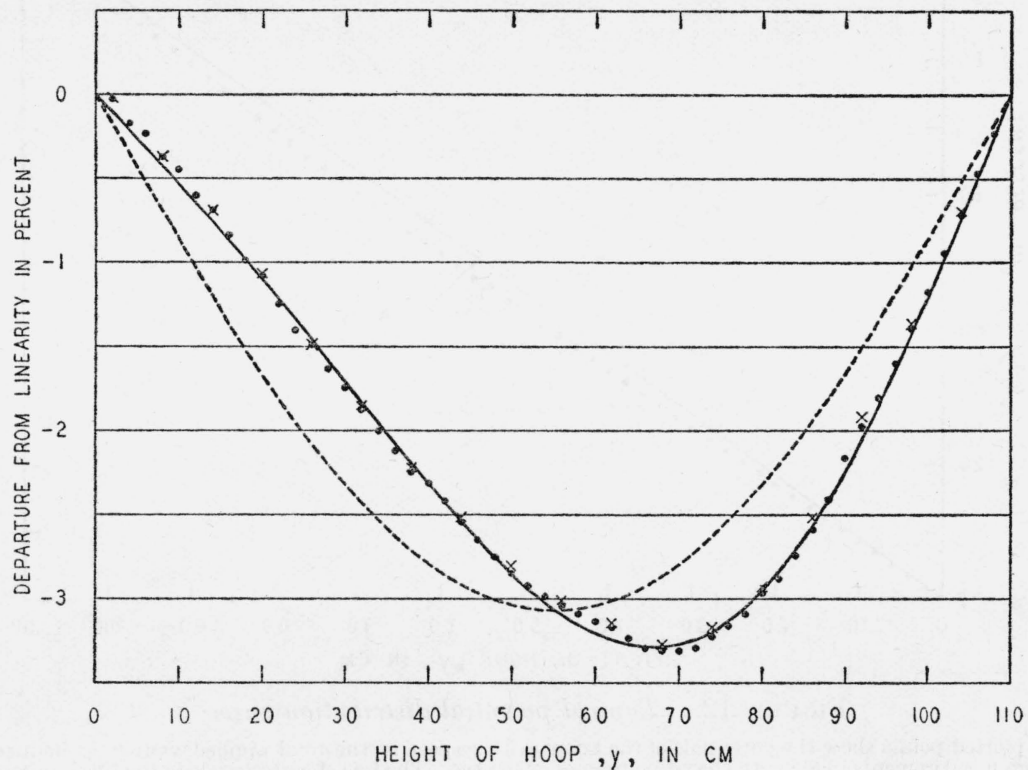

Figure 13.-Departure of hoop potentials from linearity.

Ordinates are the difference $\left(V_{S}(y) / V_{S}-y / b\right)$ between the relative potential at a height $y$ above the lower plate and that corresponding to a linear variation. A bscissas are height above the lower plate. The dots correspond to the same data as those given in figure 12; the crosses to similar data obtained when twothirds of the hoops were omitted so that the spacing was $6 \mathrm{~cm}$. The dotted curve shows the values of potential corresponding to the first term only of the Fourier series. The solid curve is that given by the first two terms of the series.

ditions is indicated by figure 13 and curve $A$ of figure 14 . In figure 13 the points show the data of figure 12 for the full separation of 110 centimeters on a greatly magnified scale by using as ordinates the difference $\left(V_{m} / V_{s}-y / b\right)$ between the observed data and the ideal straight line. The curve shows the result of summing the first two terms only of the series. Similarly, curve $A$ of figure 14 shows the resultant of the first two terms of the series using values of $G_{k}{ }^{\prime}$ computed from data taken under normal conditions with a separation of $48 \mathrm{~cm}$ between the disk and the lower plate, the normal hoop spacing of $2 \mathrm{~cm}$ being used.

\footnotetext{
${ }_{14}$ The points shown as dots were observed when the hoop spacing was the usual $2 \mathrm{~cm}$, and those indicated by crosses were observed on another occasion when two-thirds of the hoops had been removed, leaving a spacing of $6 \mathrm{~cm}$ between centers of adjacent hoops.
} 


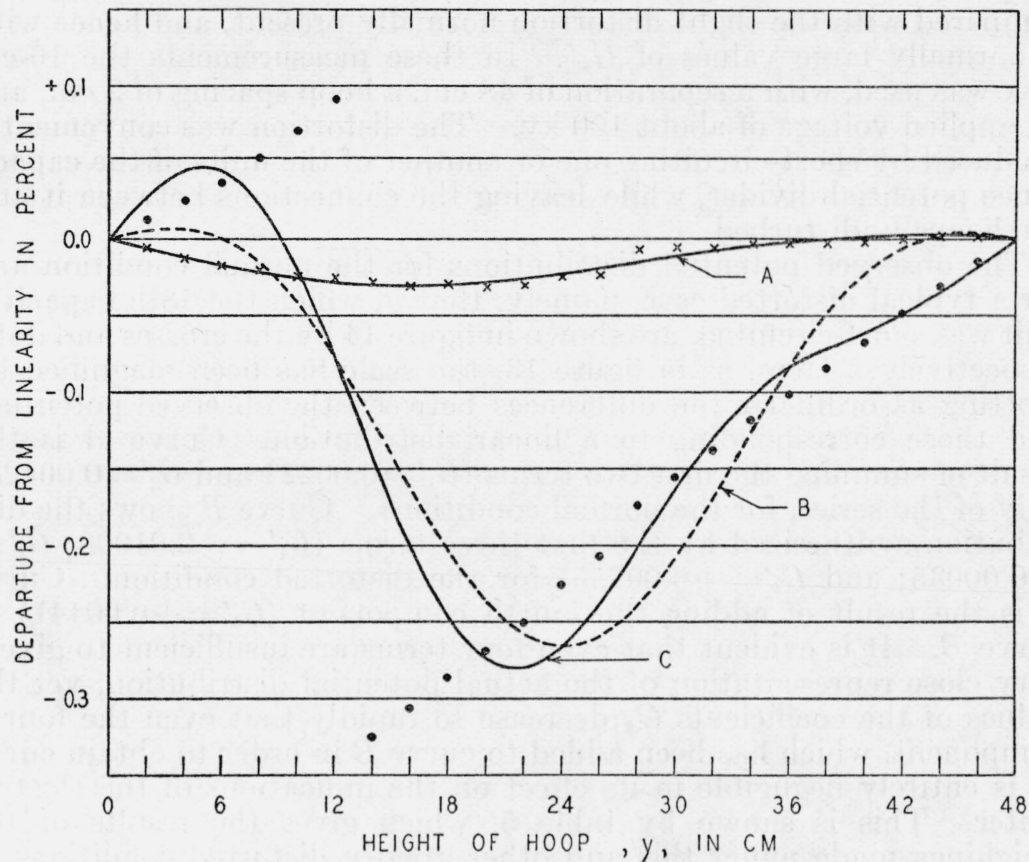

FIGURE 14.-Departure of hoop poiential from linearity under normal and abnormal conditions.

The crosses show the observed departures from linearity under normal conditions; plate separation $48 \mathrm{~cm}$ and hoop spacing $2 \mathrm{~cm}$. Curve $A$ is the resultant of the first two terms of the Fourier series based on these data. The dots show the departures from linearity observed when the 6 th and 7 th hoops, counting from the bottom, were connected together (i. e., when the 18th capacitor unit counting from the top was shortcircuited). Curve $B$ is the resultant of the first three terms of the corresponding series and curve $C$ is the resultant of the first four terms.

TABLE 4.-Typical values of Fourier coefficients and of correction for nonuniformity of hoop potentials

\begin{tabular}{|c|c|c|c|c|}
\hline \multirow[b]{2}{*}{$k$} & \multicolumn{2}{|c|}{$b=48 \mathrm{~cm} ; n=23$} & \multicolumn{2}{|c|}{$b=110 \mathrm{~cm} ; n=54$} \\
\hline & $G_{k}^{\prime}$ & $C_{k} G_{k}^{\prime}$ & $G_{k^{\prime}}$ & $C_{k} G_{k}^{\prime}$ \\
\hline${ }^{1}{ }^{1}$ & $\begin{array}{r}-0.00224 \\
-.00128 \\
-.00006 \\
\hdashline-.00663 \\
\hdashline-0.0\end{array}$ & 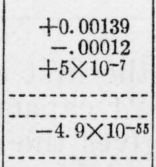 & $\begin{array}{r}-0.03099 \\
+.00655 \\
-.00026 \\
-.00014 \\
+.00072 \\
-.00290\end{array}$ & $\begin{array}{r}+0.06488 \\
+.01099 \\
+.00022 \\
+.00005 \\
-.00001 \\
-4.9 \times 10^{-58}\end{array}$ \\
\hline Sum..... & & +0.00127 & & +0.07613 \\
\hline$\Delta_{2}{ }^{2}=\ldots$ & & Negligible & & +0.00291 \\
\hline Correction factor $\mathbf{b}$.. & & 0.99873 & & 0.93042 \\
\hline
\end{tabular}

- Second order correction, as computed by eq 44.

b Factor in brackets in eq 43 .

As an experimental test of the adequacy of the theoretical correction formula, and more particularly of the correctness of the coefficients $C_{k}$, a number of weighings were made with the potential distribution deliberately distorted by an amount which was very large 
compared with the slight distortion normally present, and hence with abnormally large values of $G_{k}{ }^{\prime}$. In these measurements the $16-\mathrm{cm}$ disk was used, with a separation of $48 \mathrm{~cm}$, a hoop spacing of $2 \mathrm{~cm}$, and an applied voltage of about $100 \mathrm{kv}$. The distortion was conveniently produced by short-circuiting one or another of the units of the capacitance potential divider, while leaving the connections between it and the hoops undisturbed.

The observed potential distributions for the normal condition and for a typical distorted case, namely, that in which the 18th capacitor unit was short-circuited, are shown in figure 14 by the crosses and dots, respectively. Here, as in figure 13 , the scale has been magnified by plotting as ordinates the differences between the observed potentials and those corresponding to a linear distribution. Curve $A$ is the result of summing the first two terms $\left(G_{1}{ }^{\prime}=0.00224\right.$ and $\left.G_{2}{ }^{\prime}=0.00128\right)$ only of the series, for the normal conditions. Curve $B$ shows the distribution synthesized by the first three terms $\left(G_{1}{ }^{\prime}=-0.01908 ; G_{2}{ }^{\prime}=\right.$ +0.00035 ; and $\left.G_{3}{ }^{\prime}=+0.00755\right)$ for the distorted condition. Curve $C$ is the result of adding the fourth component $\left(G_{4}{ }^{\prime}=+0.00441\right)$ to curve $B$. It is evident that even four terms are insufficient to give a very close representation of the actual potential distribution, yet the values of the coefficients $C_{k}$ decrease so rapidly that even the fourth component, which has been added to curve $B$ in order to obtain curve $C$, is entirely negligible in its effect on the indications of the electrometer. This is shown by table 5, which gives the results of the weighings made under this and other greatly distorted conditions.

TABLE 5.-Adequacy of correction for nonuniformity of potential distribution

\begin{tabular}{|c|c|c|c|}
\hline $\begin{array}{l}\text { Capacitor unit } \\
\text { short-circuited }\end{array}$ & $\begin{array}{c}\text { Observed } \\
\text { difference } \\
\text { between } \\
\text { electrometer } \\
\text { (uncorrected } \\
\text { for hoops) and } \\
\text { voltmeter }\end{array}$ & $\begin{array}{l}\text { Correction } \\
\text { for hoop } \\
\text { potentials } \\
-\Sigma C_{k} G_{k}\end{array}$ & Residual \\
\hline $\begin{array}{l}\text { None } \\
\text { 1st } \\
6 \text { th } \\
11 \text { th } \\
18 \text { th }\end{array}$ & $\begin{array}{l}\text { Percent } \\
+0.12_{8} \\
+1.58_{7} \\
-1.04_{4} \\
-.060 \\
+1.13_{8} \\
+1.56_{6}\end{array}$ & $\begin{array}{l}\text { Percent } \\
-0.127 \\
+1.60_{3} \\
+1.077 \\
+0.07_{0} \\
-1.17_{2} \\
-1.61_{5}\end{array}$ & $\begin{array}{l}\text { Percent } \\
-0.00_{1} \\
+.01_{6} \\
+.03_{3} \\
+.00_{1} \\
-.03_{4} \\
-.04_{9}\end{array}$ \\
\hline
\end{tabular}

In this table the first column indicates which one, counting from the top, of the 24 capacitor units in use was short-circuited. The second column gives the observed percentage difference between the voltage as deduced from the electrometer without applying any correction for the potentials of the hoops and that indicated by the "voltmeter." The third column gives the value of the theoretical correction - $\Sigma C_{k} G_{k}^{\prime}$ for the same condition, the sets of values of $G_{k}{ }^{\prime}$ being computed by eq 17 from sets of data obtained with the audio-frequency bridge under both the normal and the distorted conditions, using the first three terms only. The last column gives the residual difference between the two instruments after applying the correction for hoop potentials. Each residual discrepancy is less than one-thirtieth of the uncorrected discrepancy, and the greatest residual is less than 0.05 percent. If the underlying assumptions 
on which the values of $C_{k}$ and thus of the correction $\Sigma C_{k} G_{k}^{\prime}$ are computed are sufficiently close to produce such a satisfactory correction for the large potential distortions present in these experiments, it seems that they should give a very accurate value of the much smaller correction (usually about 0.13 percent) resulting from the usual slight deviation of the potential distribution from the ideal.

As a matter of interest, a further series of experiments was carried out at a separation of $48 \mathrm{~cm}$ in which fewer than the normal number of hoops were used. The results are given in table 6 . It will be noted that when no hoops were used the electrometer was in error by over 1 percent. The presence of a single hoop, midway, reduced this error to 0.5 percent, and the insertion of more hoops rapidly diminished this error.

Similarly, when no hoops were used, the presence of a grounded metal object in the neighborhood of the instrument affected its indication by 0.2 percent, but when 11 hoops were in place the effect of this object was too small to be detectable.

TABLE 6.-Effectiveness of hoops in shaping the electric field and shielding it fro the effect of external objects

[Data with a 16-cm disk at a fixed separation of $48 \mathrm{~cm}$ ]

\begin{tabular}{|c|c|c|c|}
\hline $\begin{array}{l}\text { Hoop } \\
\text { spacing }\end{array}$ & $\begin{array}{l}\text { Number } \\
(n) \text { of in- } \\
\text { termediate } \\
\text { hoops used }\end{array}$ & $\begin{array}{l}\text { Excess of } \\
\text { electrometer } \\
\text { indication } \\
\text { over that of } \\
\text { "voltmeter" }\end{array}$ & $\begin{array}{l}\text { Increase in } \\
\text { electrometer } \\
\text { indication } \\
\text { due to ex- } \\
\text { ternal object } 1\end{array}$ \\
\hline $\begin{array}{c}\text { Centi- } \\
\text { meters } \\
2 \\
4 \\
6 \\
8 \\
12 \\
16 \\
24 \\
48\end{array}$ & $\begin{array}{r}23 \\
11 \\
7 \\
5 \\
3 \\
2 \\
1 \\
0\end{array}$ & $\begin{array}{c}\text { Percent } \\
0.00 \\
.03 \\
.13 \\
.17 \\
.26 \\
.36 \\
.48 \\
1.06\end{array}$ & $\begin{array}{r}\text { Percent } \\
0.00 \\
+.01 \\
+.04 \\
+.05 \\
+.10 \\
+.12 \\
+.18\end{array}$ \\
\hline
\end{tabular}

1 The "object" consisted of a grounded metal hemisphere $20 \mathrm{~cm}$ in diameter, mounted at the level of the lower plate at a distance of $38 \mathrm{~cm}$ from the hoops.

In the preceding discussion it has been assumed that the hoops were placed at exactly their proper heights and that only the potentials could be incorrect. This condition is fairly closely approached in the actual construction. Examination of a large number of measurements of height indicates that the hoops were on the average too high by $0.01_{1} \mathrm{~cm}$; the average deviation of these measurements, without regard to sign, being $\pm 0.02_{7} \mathrm{~cm}$.

It can be deduced from eq 17 that an error $(\Delta y)_{m}$ in the height of the $m$ th hoop produces an error in voltage given by

$$
\frac{2 s}{b^{2}}(\Delta y)_{m} \sum_{k=1}^{k=\infty} C_{k} \sin \frac{k m \pi}{n+1}
$$

where $s$ is the hoop spacing $(2 \mathrm{~cm}), n$ is the number of intermediate hoops in use, and $b$ is the separation between disk and lower plate. From the values of $C_{k}$ given in table 3 , it appears that to produce an error of 0.01 percent, even under the worst conditions, one hoop would have to be as much as $1 \mathrm{~mm}$ out of place. However, a systematic 
shift of all the hoops by $1 \mathrm{~mm}$ would produce an error of $0.02_{5}$ percent at a separation of $24 \mathrm{~cm}, 0.16$ percent at a separation of $48 \mathrm{~cm}$, and 0.10 percent at $110 \mathrm{~cm}$. Hence in the most careful work at these larger separations, corrections based on measurements of hoop height in which the accuracy of the mean is better than $0.1 \mathrm{~mm}$ are necessary.

It will be shown in section IX-2 that a further source of error exists in the possibility that the presence of external objects, and even of the floor and walls of the room, may cause the potential of a point in the space between the hoops to be appreciably different from the value it would have if the potential gradient external to the hoops was purely axial. Any such effect will be greatest when the plate separation is greatest, and will also increase rapidly with the spacing

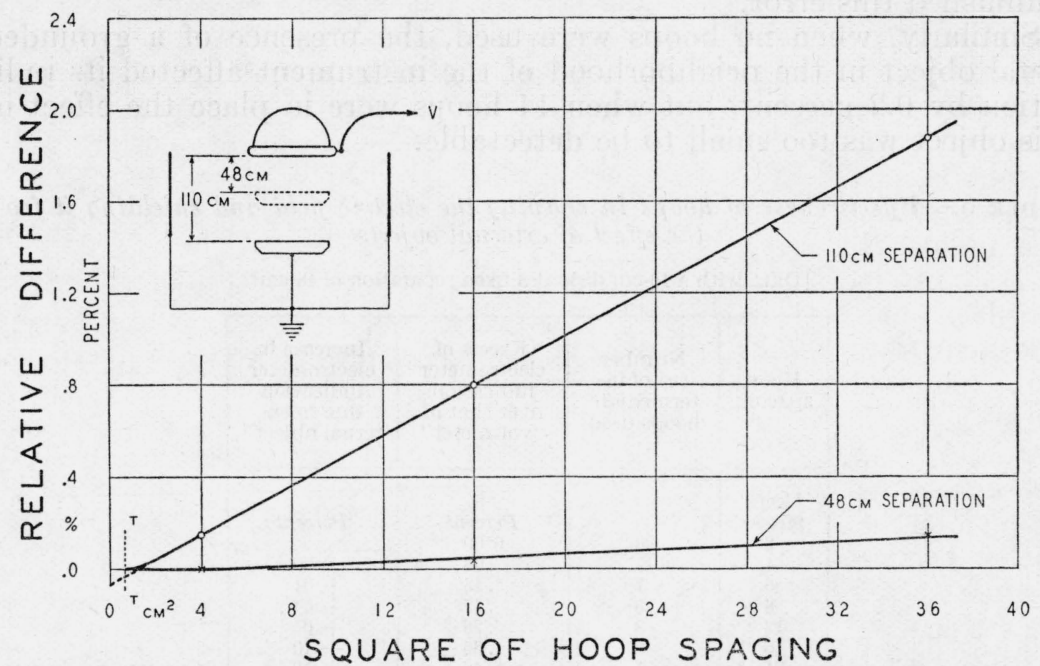

FIgURE 15.-Effect of distortion of the electric field between the hoops

Ordinates are the relative differences in the indications of the electrometer and of the "voltmeter." Abscissas are the squares of the center-to-center spacings of the hoops. The true indication should correspond to a spacing intermediate between the smallest, $2 \mathrm{~cm},\left(s^{2}=4\right)$ at which observations were made and that, indicated by the line $T T$, at which the hoops touch. 'The insert shows to scale the electrometer and at each side the grounded metal of the walkways.

of the hoops. A rough estimate indicates that at the full separation of $110 \mathrm{~cm}$ this effect, if uncorrected, might introduce an error of 0.13 percent and that at a hoop spacing of $6 \mathrm{~cm}$, instead of the normal $2 \mathrm{~cm}$, this error would increase to 1.4 percent.

A series of experiments was made to test the correctness of these estimates by making measurements at a separation of $110 \mathrm{~cm}$, first with the normal hoop spacing of $2 \mathrm{~cm}$ and then with part of the hoops removed to make the spacing $4 \mathrm{~cm}$ and later $6 \mathrm{~cm}$. The results are shown in figure 15. Here each ordinate is the difference, expressed in percent, between the indications of the electrometer and of the "voltmeter," and the abscissa is the square of the hoop spacing. The insert shows in true proportion the relative distances between the plates and walkways. ${ }^{15}$ It will be seen that the change in error with hoop spacing is in reasonable agreement with the estimated values.

\footnotetext{
16 Two semicircular wooden walkways (faced with a grounded metallic mesh) are used, on which the observer stands while gaging and adjusting the balance mechanism. They are moved as far as possible away from the electrometer before voltage is applied but are then at a relative spacing indicated by the insert in figure 15. All other metal objects in the neighborhood of the electrometer are also grounded.
} 
The lower curve in figure 15 gives corresponding data obtained with a plate separation of $48 \mathrm{~cm}$. At this smaller separation the ratio of the radial component of the external potential gradient to the axial component is much smaller than at $110 \mathrm{~cm}$, and the coefficients $C_{k}$ are also much smaller, so that even if the same error in $G_{k}{ }^{\prime}$ were present it would produce much less error in voltage. Consequently, the excess indication at $2-\mathrm{cm}$ spacing and $110-\mathrm{cm}$ separation over that at $48-\mathrm{cm}$ separation may be taken as a correction, and applied in the later use of the instrument at $110 \mathrm{~cm}$ under the same geometrical condition, even at higher voltages than can be applied safely at the shorter spacing. This correction we have designated as $\phi_{2}\left(s / r_{h}\right)$ in eq 12 . It is expected that as soon as greater space can be provided for the electrometer, enabling the walkways and other grounded objects to be removed to greater distances, this effect will be much reduced. By the addition of properly shaped conducting elements to the guard ring this effect can be further reduced to almost any desired extent. The criterion for the proper condition which gives a very small radial component of field will be the vanishing of the slope of lines such as those of figure 15, in which electrometer indication is plotted against the square of the hoop spacing.

It is interesting to note from figure 13 that the omission of twothirds of the hoops to give a spacing of $6 \mathrm{~cm}$ did not appreciably change the potentials of the hoops which were retained, and therefore did not change the value of $G_{1}{ }^{\prime}$ computed from the hoop potentials by more than 0.0004 . The weighings, however, indicate that the true value, $G_{1}$, which includes the effects of the potential of points between the hoops, was probably changed by nearly 0.008 , and hence that the ripple had an amplitude of approximately 0.8 percent. Ripples of this rather extreme magnitude are shown in case $I I D$ of figure 24 .

In conclusion, the efficacy of the hoops may well be illustrated by an experiment made with a separation of $110 \mathrm{~cm}$ and with no intermediate guard hoops, which showed that the voltage required to produce a given force was then only 71 percent of that required when conditions were normal. The instrument without its hoops thus has an error of 41 percent of the true voltage being measured, as contrasted with a correction of about 0.1 percent when the hoops are used at this largest separation.

\section{FLATNESS OF LOWER PLATE}

When used at small separations the flatness of the lower plate is presumably of almost equal importance with that of the disk and of the guard ring in determining the density of charge and hence the force of attraction. As the separation is increased, however, the guard hoops become of greater and the lower plate of less importance in determining the electric field at the disk. At a separation of $110 \mathrm{~cm}$, the removal of the guard hoops was found to change the calibration by 41 percent. A further indication of the lessened importance of the lower plate was obtained by an experiment at a separation of $48 \mathrm{~cm}$ with the hoops in the normal spacing and connection. A disk of metal, $1 \mathrm{~cm}$ thick and $11.2 \mathrm{~cm}$ in diameter, was placed on the center of the lower plate directly beneath the attracted disk. The voltage then required to produce a given force was found to be only 0.31 percent less than that required when the extraneous disk was absent, although the reduction in separation at the center was 2.1 percent. 


\section{VIBRATION}

The pulsating character of the electrostatic force must cause a certain very small vertical oscillation in the disk of the order of $10^{-5}$ $\mathrm{cm}$. The vibration of the balance mirror resulting from this is too small to be visible unless some resonant condition is approached. For one condition of loading for low stability, the mirror and stabilizing arm were found to exhibit a resonant vibration at 62 cycles per second, which produced a widening of the image at the scale. This widening was found experimentally not to alter the value of voltage determined by the electrometer, but since it reduced the ease of reading it was eliminated by loading the mirror mounting and thereby reducing the resonant frequency to about 55 cycles per second.

Because of the variation in instrument "constant" with height of the disk any appreciable vibration will tend to introduce a net contribution to the average force. Calculations indicate that such effects should not exceed a few thousandths percent unless the vibration is so large as to be easily visible.

Since it is assumed that the measured force is of electrostatic origin only, it was felt that a test should be made to determine the presence of any spurious aerodynamic force on the disk, such as the Bjerknes effect. The immediate concern was with continuous vibrations, either coming as sound from the 60-cycle supply transformers and electric heaters in the laboratory, or occurring in the disk itself because the electric force is pulsating. That such an effect is unimportant was determined by placing an improvised loud-speaker directly below the attracted disk and producing sound vibrations, over a wide range of audio frequencies, of several thousand times the amplitude usually present in the laboratory. Under this drastic condition only a small deflection was observed, and this was due largely to a translation of the balance beam resulting from its excessive vibration, the rotation being negligibly small.

The electrometer is not especially sensitive to the usual vibrations from nearby machinery and traffic.

\section{MECHANICAL DETAILS}

\section{MAIN STRUCTURE}

The main framework of the electrometer, as shown in figures 2 and 27 , consists of a circular base (43), an upper circular plate (4) with a central opening, and an intermediate ring (42), all about 1 meter in diameter. These parts are rigidly united by six hollow pillars (36) of fused silica $2.1 \mathrm{~m}$ long. The three metal parts were made of a particular cast iron chosen for its high machinability, stability, and freedom from warping, and were annealed before delivery to the Bureau. After the lower surface of the upper plate was machined, ${ }^{16}$ it was "spotted" on a precision surface plate, then stored for a period of months and again checked against the surface plate. This repeat test showed that the casting had not warped an appreciable amount during the period of storage, and the lower surface was accordingly then scraped to a plane.

${ }_{16}$ This work was very carefully done by the U. S. Naval Gun Factory at Washington, the machine-tool equipment of the instrument shop of the National Bureau of Standards being inadequate to machine pieces of this size and weight. 
The four cast-iron bushings (3) which make it possible to use any one of four attracted disks of various diameters, were machined to fit accurately into the circular central opening $(30.2 \mathrm{~cm}$ diameter) in the upper plate. These bushings are of iron of the same quality as the main castings. Each bushing was accurately scraped while in the upper plate so that its lower surface would be in the same plane as the lower surface of the upper plate. The upper surface of each bushing was ground accurately plane and parallel to its scraped lower surface, in order that its definite thickness could be used in measuring the separation of the opposed surfaces of the lower plate and of the guard ring. Since the flatness and surface condition of that portion of the guard ring closely adjacent to the attracted disk are more important than those of the remainder of the guard ring, this portion was formed of a stainless-steel ${ }^{17}$ insert (2) having a width of several centimeters. After being machined, the vertical side of the central opening of the insert and its lower surface were lapped and polished. The insert was held in position from above by retaining screws and stop screws which permitted precise vertical adjustment of the lower surface of the insert to make it coincide with the plane of the surrounding surface of the bushing.

The bushings were made hollow, primarily to avoid unnecessary weight, but the hollow interior proved to be of great convenience in the equalization of air pressure on the two sides of the disk. Six holes $20 \mathrm{~mm}$ in diameter extend completely through each bushing to accommodate the gage rods used when measuring the separation between guard ring and lower plate. These holes are ordinarily completely closed at the upper side and partially closed at the lower or working surface by means of brass plugs (9). The lower face of each plug was machined so as to lie in the plane of the lower surface of the bushing. The bottom and sides of these plugs are perforated so as to allow free passage of air between the lower side of the disk and the interior of the bushing.

The intermediate ring carries three micrometer screws (40), $120^{\circ}$ apart, and between them three jackscrews (41) for raising or lowering the lower plate when it is to be set at a new level. At six equidistant points the cross section of the ring is enlarged to admit openings through which pass the silica pillars. The ring is rigidly fastened by screws to iron sleeves which in turn are secured to the pillars by casting melted tin in the small annular space between the sleeves and the pillars. Cast-iron machined sockets are attached to the ends of the pillars by the use of tin cast in place, and the sockets are secured by cap screws to the upper plate and to the lower plate.

Fused silica was chosen for the pillars for two principal reasons, namely, its very low expansivity and its high electrical resistivity. It retains its high surface resistivity very well under the condition of high relative humidity of the air. It has obvious minor drawbacks, such as the difficulty of obtaining it in regular geometric forms and of machining it. Its most serious shortcoming for the present purpose is its fragility. The upper plate of the electrometer, weighing about 600 pounds and bearing the intricate and expensive balance mechanism, is carried at a height of about 8 feet from the floor on six brittle pillars, one or more of which might easily be broken by

${ }_{17}$ This close-grained material (20 percent of $\mathrm{Cr}, 1$ percent of $\mathrm{Cu}$, and 0.30 percent of $\mathrm{C}$ ) is capable of being worked by optical methods to a high precision. 
an inadvertent blow. To safeguard personnel as well as the electrometer mechanism, each hollow silica pillar has an inner pillar of thick-walled Micarta tubing (see (36) and (37), fig. 27). Because of the relatively high expansivity of the Micarta as compared with that of the fused silica, this inner pillar was made a little shorter than the silica pillar so as to take none of the load under ordinary conditions. There is a clearance between the Micarta pillar and the silica pillar which avoids any reduction in the surface resistance between the hoops.

Three of the pillars, spaced $120^{\circ}$ apart, have 55 small holes drilled in each, $2 \mathrm{~cm}$ apart, to receive pegs (32) for supporting the guard hoops (31). These holes were drilled by using a brass tube guided by a steel jig and kept charged with a mixture of water and carborundum.

\section{LOWER PLATE}

The lower plate (35) was cast of a special aluminum alloy ${ }^{18}$ having a hardness about equal to that of cast iron. This alloy was used to make the plate light and easy to adjust when the distance between it and the upper plate is changed. To give maximum rigidity for a given mass of metal, the lower plate is heavily ribbed, after the manner of the precision surface plates used by machinists. Its upper surface was scraped to be accurately plane.

To protect the upper surface of the lower plate during gaging and when the instrument is not in use, an aluminum cover $6 \mathrm{~mm}$ thick is provided. This is formed of three interlocking pieces to permit its insertion between the pillars.

In use the lower plate rests on a set of three rods (38) (fig. 27) of nickel-steel alloy of low expansivity. ${ }^{19}$ At their upper ends these rods are drilled and threaded to screw to studs set $120^{\circ}$ apart in the under side of the lower plate, near its edge. The lower ends of these three legs are connected rigidly by a triangular aluminum frame (39) clamped to them by setscrews.

The ends of the three rods and of the micrometer screws on which they rest, were shaped in accordance with the classical "hole, slot, plane" arrangement. This permits the plate to be lowered to a definite position on the micrometer screws without introducing any strain or deformation. Unfortunately, the weight of the plate is so great that the resulting wedging of one screw in the slot and the friction of the other on the plane prevent the sliding required to relieve strain from differential thermal expansion. The relatively slender rods can bend sufficiently, however, to relieve such strain without developing any appreciable distortion in the lower plate itself.

The bearing surfaces of the rods and the adjusting screws (40) were chromium-plated to minimize wear. Each adjusting screw covers a range of somewhat more than $10 \mathrm{~cm}$ and the sets of supporting rods differ in length by a constant interval of $10 \mathrm{~cm}$. The screws are of $1-\mathrm{mm}$ pitch and their heads are graduated to read directly to $0.01 \mathrm{~mm}$.

The three jackscrews (41) have coarse threads to permit relatively

\footnotetext{
18 This plate was cast, machined, and scraped at the Naval Gun Factory. The alloy used had an expansivity of $21 \times 10^{-6}$ per ${ }^{\circ} \mathrm{C}$.

${ }_{10}$ A bout $2 \times 10^{-6}$ per ${ }^{\circ} \mathrm{C}$. Although nickel-steel alloy of still lower expansivity was obtainable, its much higher cost prevented its use.
} 
rapid change of level of the lower plate. They also serve as a safety feature, for although they are lowered slightly after the plate reaches the desired level, to let the weight be carried on the micrometer screws, the ends of the jackscrews project deeply into recesses in the under side of the lower plate.

\section{DISKS AND DAMPING CHAMBER}

The attracted disks were made of sheet duralumin $1.5 \mathrm{~mm}$ thick. A central hub carrying a small conical chuck (see $a$, fig. 16) is attached to each disk by four screws which are so short as not to extend through the disk and mar its lower surface. This chuck grips the stem, which is made of thin-walled phosphor-bronze tubing, $2.5 \mathrm{~mm}$ in diameter and $30 \mathrm{~cm}$ long. At its upper end the stem has a conical fitting which engages a cone which in turn hangs on an annealed-copper suspension strip $0.6 \mathrm{~mm}$ by $0.03 \mathrm{~mm}$ by $4.0 \mathrm{~cm}$ long. The upper end of this strip is attached to the stirrup carried by the end agate bearing of the balance. The longer dimension of the cross section of the suspension strip is at right angles to the axis of the knife-edge so that the disk can hang as a pendulum without constraint in either direction, although any rotation about a vertical axis is checked by the torsion of the suspension. The rigid stem was made long to insure that the disk would hang nearly level even though the line from the center of the conical fitting perpendicular to the plane of the disk does not pass exactly through the center of gravity of the disk.

Disks of approximately the correct diameter were made to the required flatness or curvature as follows: A disk was clamped between two lapped and polished stainless-steel plates having the desired flatness or curvature and lubricated with graphite; this assembly with a thermocouple was packed in asbestos and held at approximately $400^{\circ}$ $\mathrm{C}$ for 7 hours, then allowed to cool slowly. Both sides of the disk were lapped and polished with chromium oxide, the two sides being worked alternately. When necessary the disk was reannealed. Subsequently a light finishing cut was removed from the edge. The lower side of the disk was then ruled with a pattern of 25 extremely shallow radial lines $0.2 \mathrm{~mm}$ apart at the circumference and about $4 \mathrm{~mm}$ long, and other single lines at $60^{\circ}$ intervals from the main group of lines. All burrs were then removed by honing with a fine abrasive stone. The main group of 25 lines is used in locating the disk in azimuth relative to a fiducial line on its bushing, when the disk is suspended in its working position in the electrometer. From the azimuth as thus located and the curve showing the height of the rim of the disk at various azimuths obtained when it was surveyed, its mean height can be correlated with the heights of the six positions on which the coplanarity microscopes are sighted.

Early experience with the electrometer showed that serious disturbances were produced even by very slow air currents, such as those resulting from temperature inequalities in the laboratory and within the electrometer dome, and even by wind outside the building. To minimize these effects, the air space just above the disk is separated as much as possible from the air in the dome by a metal cover $a$, figure 17. The stem of the disk passes through a hole in the center of this cover with only the necessary small clearance. The volume of the confined air space above the disk is reduced to a minimum by a wooden 
filler (8), figure 27 , and this space is vented to openings in the under surface of the guard ring. These openings are formed by small perforations ( $4 \mathrm{~mm}$ in diameter) in the lower ends of the brass plugs (9), figure 27 , which normally close the six holes which are provided in each bushing for use in gaging. Because of the symmetrical location of these holes, the mean air pressure at them must be closely equal to the mean pressure at the disk. This mean pressure is transmitted through the plugs to the hollow interior of the bushing and thence through two connecting tubes $b$, figure 17 , and (10), figure 27 , to the air space behind the disk. A convenient adjustment of the damping is obtained by the use of a butterfly valve in one of these connecting tubes. This was usually so adjusted that the motion was slightly underdamped.

\section{BALANCE}

The balance beam and the stirrups used in the electrometer were taken from an assay balance. The beam is made of aluminum alloy and provided with agate knives. All three bearings are also of agate, the central one being plane and the end bearings of the $V$ type. The load at either end is about $100 \mathrm{~g}$. The distance between the end knife-edges is $128 \mathrm{~mm}$. In the electrometer, the requirements to be met by the arrestment mechanism are so much more severe than those attending the ordinary use of a balance that an attempt to improve and adapt the control mechanism furnished by the balance maker was very soon abandoned, and a completely new design was developed. A view of this from the rear is shown in figure 18. Figure 19 shows a front view of part of the mechanism beneath the balance base; this latter view does not show the balance beam. Figure 20 shows a side view of the indicating and control mechanism.

The balance base (24), figure 27, is of annealed cast iron, and is supported from the upper plate by three legs (25). Micrometer adjustment provided at the upper ends permits the upper surface of the base to be brought into the desired plane. To minimize the vertical shift with temperature of the disk relative to the lower surface of the guard ring, the lower part of each leg is made of aluminum and the upper part of steel, as may be seen from figure 19; the proportionate lengths of the two parts are chosen with reference to the thickness of the cast-iron balance base and the height of the phosphor-bronze balance column from the upper surface of the base to the level of the central agate plane, so that the resultant expansivity of the structure supporting the balance beam shall be equal to the expansivity of the phosphor-bronze tubular stem and the suspension strip of the attracted disk.

Stop screws and clamping screws provide for locating and securing the balance base in approximately its correct position, namely, that in which the attracted disk hangs freely in the center of the opening in the guard ring. To allow the necessary horizontal adjustments the upper end of each leg fits into a hole in the underside of the balance base, with a relatively large clearance.

To provide the final, very precise horizontal adjustment of the position of the disk, the balance column is carried on a double $V$-slide arrangement with the two slides at right angles (fig. 17). The heads $c$ of the screws controlling these slides are graduated in divisions, each corresponding to a movement of $0.01 \mathrm{~mm}$ of the balance column. 


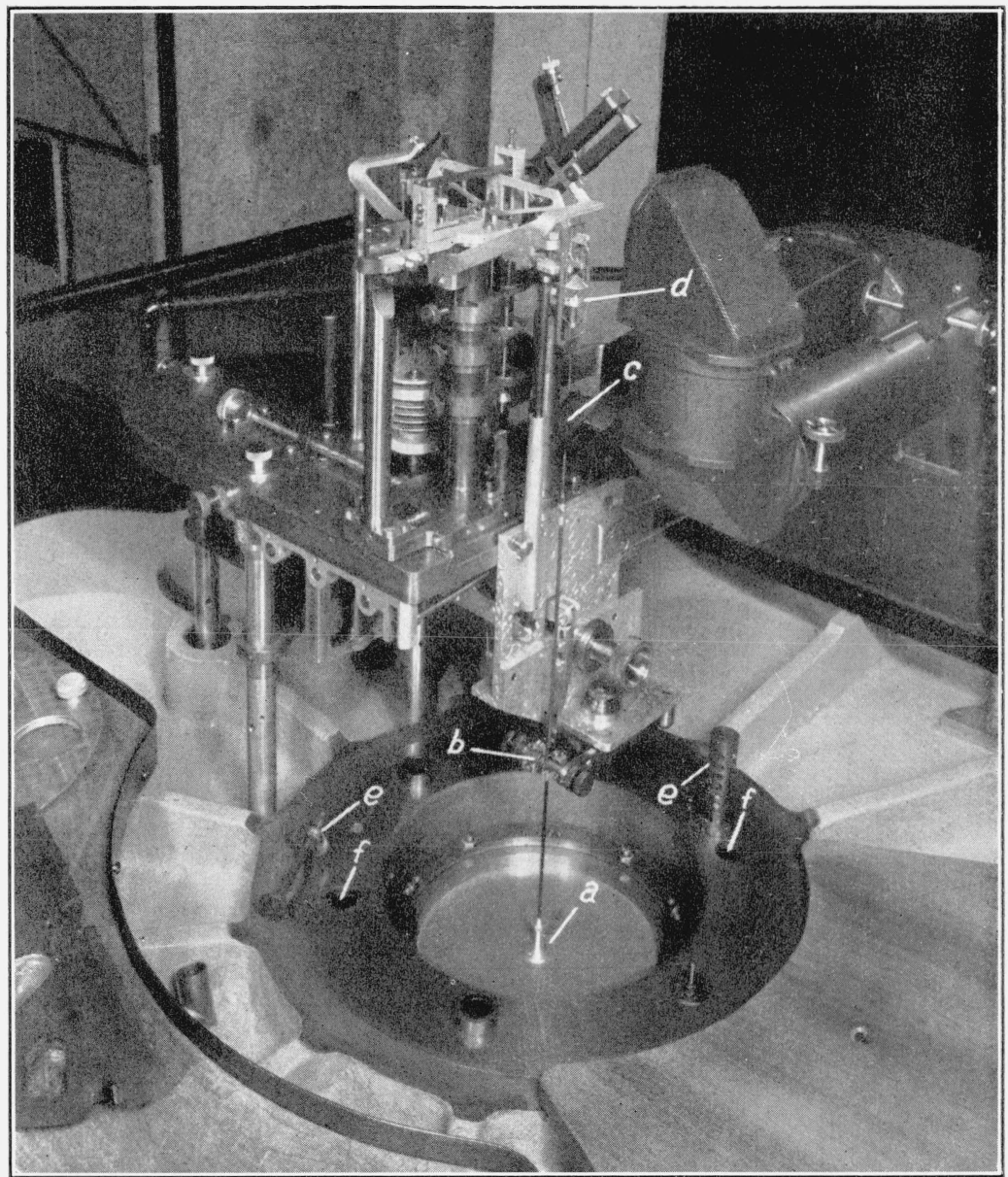

FIgURE 16.-View looking down at upper side of disk, in position.

Showing the hub, $a$; the stem and its clamp, $b$; the suspension strip, $c$; weights on the weight pan, $d$; and also the two types of perforated plugs, $e$, used in the gaging holes, $f$. 


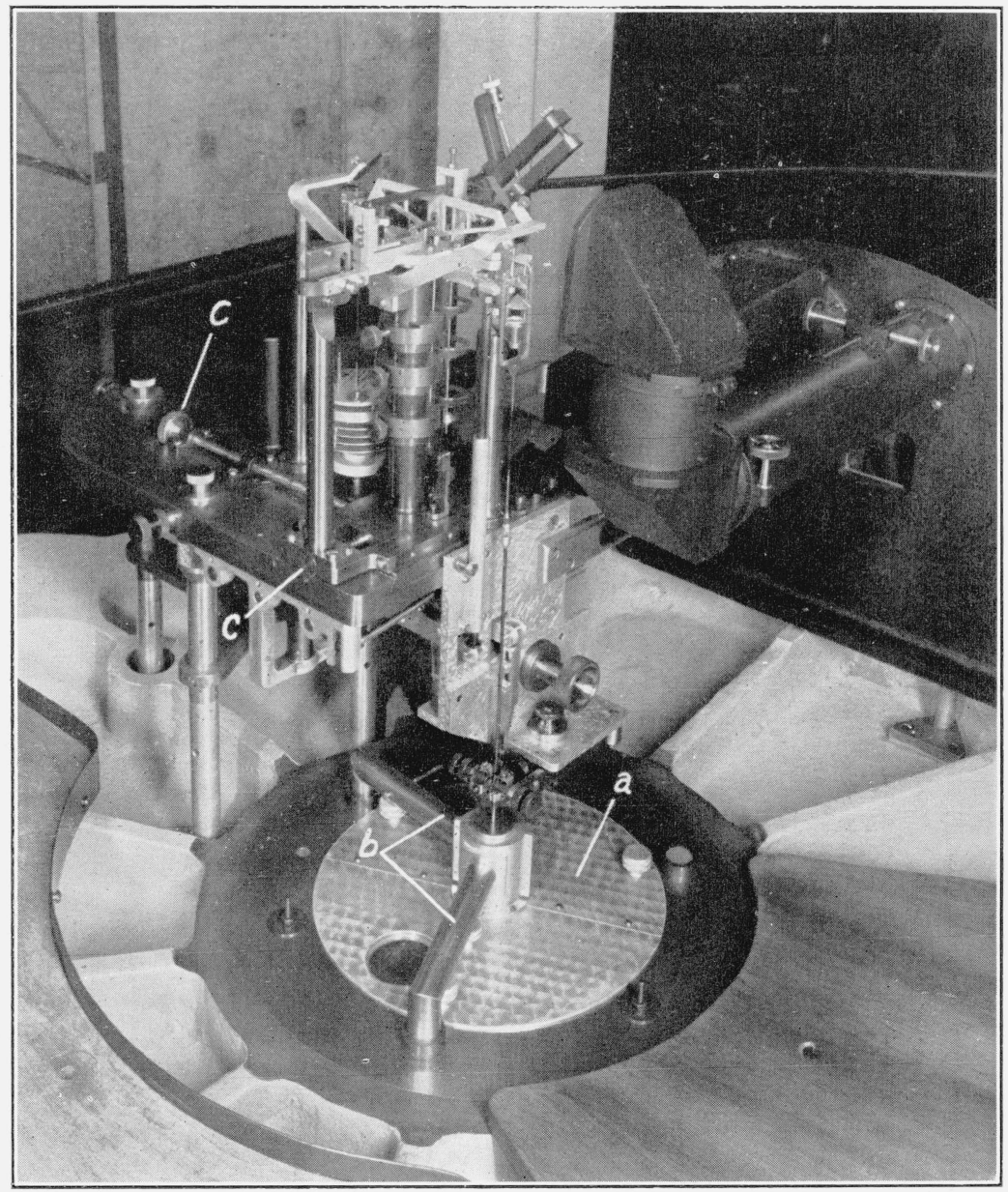

FIGURE 17.-View of balance mechanism.

Showing the metal cover, $a$, above the disk; the tubes, $b$, connecting air space above it to the gaging holes; and the heads, $c$, of the micrometer screws used to adjust the balance column in centering the disk. 




FIGURE 18.-Balance mechanism as seen from the side opposite the observer's station.

The counterpoise, $a$, with its multiple aluminum disks, and the chainomatic device, $b$, appear at the left of the balance pillar.

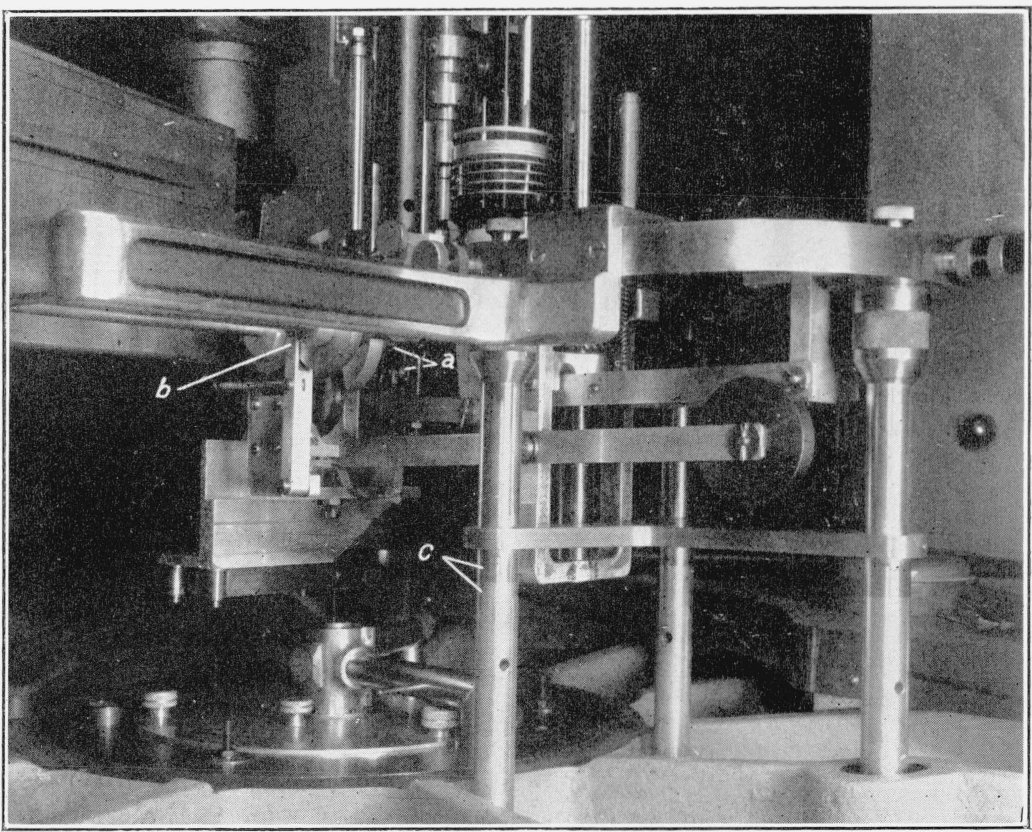

FIGURE 19.-Lower part of balance control mechanism, showing cams, a; interlock, $b$; and bimetallic balance base legs, $c$. 


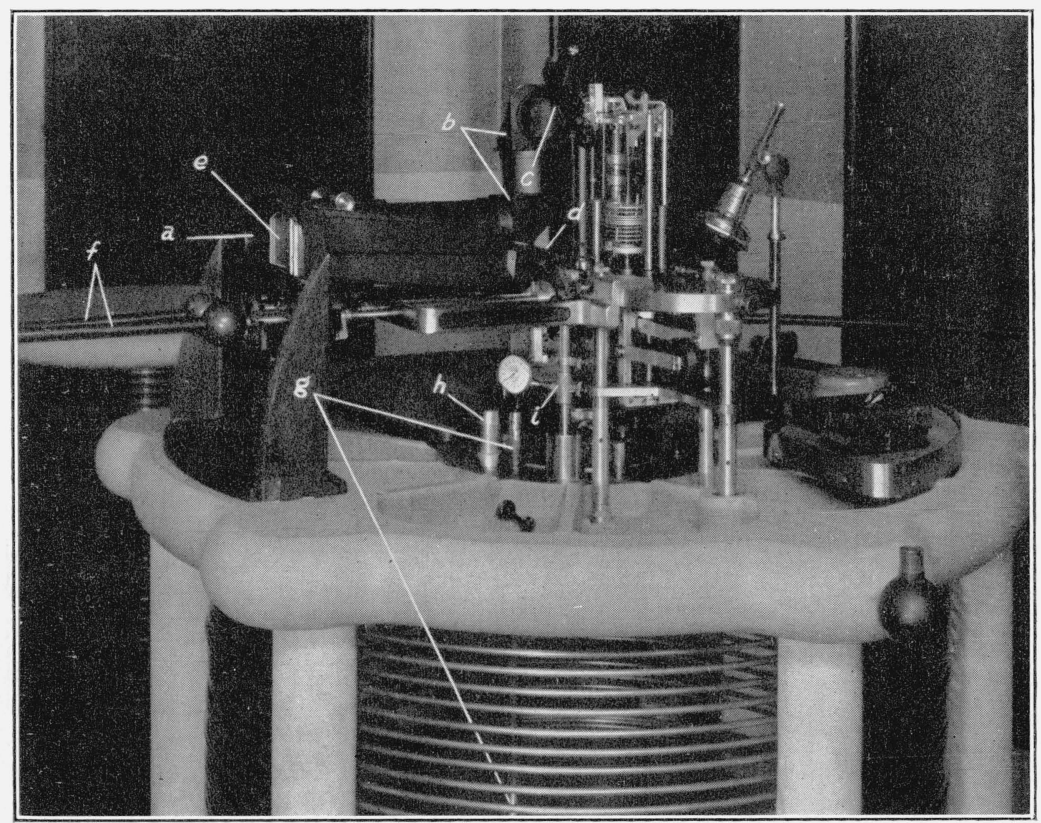

FiguRE 20.- Side view of electrometer mechanism with dome lifted.

Light from the source enters the light tube at $a$ and is reflected by prisms, $b$, to the mirrors, $c$. Thence it is reflected by the prism, $d$, through the exit window, $\rho$, to the ground-glass scale at the observer's station. Control rods, $f$, at the left extend to the observer's station (not shown). Gage rod, $g$, block, $h$, and surface gage, $i$, are in position for measuring separation. 
In the control mechanism for the balance a main arrestment is provided to remove the loads from the three knife-edges when the electrometer is not in use. This arrestment must also be operated between the determination of the disk height $h_{m}$ and the actual weighing of the electrostatic force. In order that this operation of the mechanism shall not alter the previously determined correlation between disk height and scale reading, it is a fundamental requirement that each time the balance is released the disk shall return to the same position. For a precision of 0.01 percent the permissible tolerance in the vertical height of the disk is of the order of $1 \mu$, and a motion, even if horizontal, of one end of the beam exceeding this amount will also produce an angular motion of the mirror which will introduce an appreciable error in the scale indication.

The main arrestment utilizes the classical "hole, slot, plane" principle. The conical "hole" is located near that end of the beam which carries the disk, so as to return the disk always to the center of the aperture. The "slot" is at the other end of the beam, and the "plane" is formed on an extended part of the mirror mounting in line with the central knife-edge. Individual adjustments are provided for the two $60^{\circ}$ lifting cones which engage the hole and the slot and for the spherical segment which engages the plane. The mechanism just described serves to lift the balance beam or to replace it on the central agate plane. Another lifter carrying two adjustable cones engages a hole and a slot in the upper part of the stirrup which carries the disk. In the fully arrested position this lifter separates the agate bearing from the knife-edge at this end of the beam. The load is taken off the third knife-edge in a more conventional manner by an adjustable plunger under the counterpoise which lifts it enough to take most of the weight. All three parts of the main arrestment are operated by turning a single control rod, and are so adjusted that the beam with its central knife-edge is lifted first and the end bearings immediately afterward. On release, these operations occur in the reverse order.

As a test of the precision with which the beam arrestments performed their function, six pairs of determinations of the zero of the instrument were made. Each determination consisted of 30 readings taken at intervals of about 3 seconds and averaged to eliminate the effect of variations in the ever-present air currents. After the first determination of each pair, the balance was arrested and released and the second determination of the pair was made. The six pairs of determinations were made on different days and the mean of the differences (taken without regard to sign) between the members of each pair was found to have the surprisingly small value $0.0011 \mathrm{~cm}$ on the scale. The balance sensitivity was such that this difference corresponds to a change in weight of less than 2 parts in $10,000,000$ or to a shift in the effective length of the balance arm of only $0.01 \mu$.

However, the mass $M_{F}$, which is a measure of the attractive force, is at best only one-thirtieth of the total mass of the disk, stem, etc., so that an error in voltage of 0.01 percent will be produced by a change of only 2 parts in 1,000,000 in the effective length of either arm of the balance. Because the above very gratifying performance had not been anticipated, the possibility of errors from this source had been minimized by providing, in addition, a stem-clamping device ${ }^{20}$ which serves to grip the stem of the disk while the mass $M_{p}$

${ }^{20} \mathrm{~J} . \mathrm{H}$. Poynting. On a method of using the balance with great delicacy and on its employment to determine the mean density of the earth. Trans. Roy. Soc. (London) 28, 2 (1878). 
or the voltage is being applied or removed. The purpose of this device (12), figure 27 , is to ensure that the knife-edges remain in undisturbed contact with their bearings throughout the weighing process. The stem clamp consists of a pair of flexible jaws which are pressed together by a spring and moved apart by a cam. A very light pressure is sufficient because the weight to be changed never exceeds a few grams. The jaws are adjusted so that the unavoidable impulses of translation or rotation which they tend to impart to the stem as they release it are very small.

Two leaf-spring stops are located above the balance beam and adjusted to limit its motion to correspond to the length of the groundglass scale at the observer's platform. By thus restricting the motion of the beam the relative shift of the balance knife-edges and bearings resulting from large deflections is minimized.

Interlocks are provided so that the main arrestment cannot be operated unless the stem clamp is in its released position, and the clamp cannot be operated unless the main arrestment is released. The same handle controls both the stem clamp and the lifter for the weight pan, so that the weights cannot be changed except while the stem is clamped.

For denominations of $20,50,100 \ldots 1,000 \mathrm{mg}$ the weights used are small cylinders of platinum-iridium alloy, which was chosen because of its high density and freedom from corrosion. The weights have rounded edges and a height 1.5 times the diameter. An electrical-instrument pivot, mounted in a pantograph engraving machine, was used as a marking tool to burnish the numerals $0.4 \mathrm{~mm}$ high on the top of each weight. This method of marking produces no grooves in which dirt may collect. The weight pan, of an aluminum alloy, contributes $100 \mathrm{mg}$ to the total removable weight $M_{p}$.

A fine, continuous adjustment of the load on the counterweight arm of the beam is conveniently made from a distance by means of a mechanism which we have designated by its trade name, "chainomatic." It may be seen at the end of the balance beam in figure 18 . One end of a gold chain of very small links weighing $0.029 \mathrm{~g} / \mathrm{cm}$ is attached to a metal cup in which is mounted an inverted cupped sapphire jewel, which rests on the polished, rounded end of a steel pivot carried by an arm attached to the balance beam near its end. This arrangement allows the utmost flexibility of motion of the chain while maintaining a very definite length of arm at which the weight of the chain acts. The outer end of the chain is attached to a horizontal arm carried at the top of a vertical plunger which can be raised or lowered slowly by rotating a shaft at the observer's station. The convenient fine adjustment of the counterweight from a distance, made possible by this chainomatic device, would obviously involve great difficulty if it were attempted by the ordinary process of moving a rider.

Figure 21 shows the balance beam $c$, the beam mirror $a$, and alongside it the fiducial mirror $b$ carried by a rigid support from the balance base and capable of fine adjustment by three screws. The light source at the observer's station consists of a brightly illuminated reticle, which is a photographic negative of a scale consisting of equally spaced lines. Light from this source enters the electrometer through a tube ( $a$, fig. 20) which contains a lens adjustable by a rack and pinion for focusing. It is reflected upward and to the right 


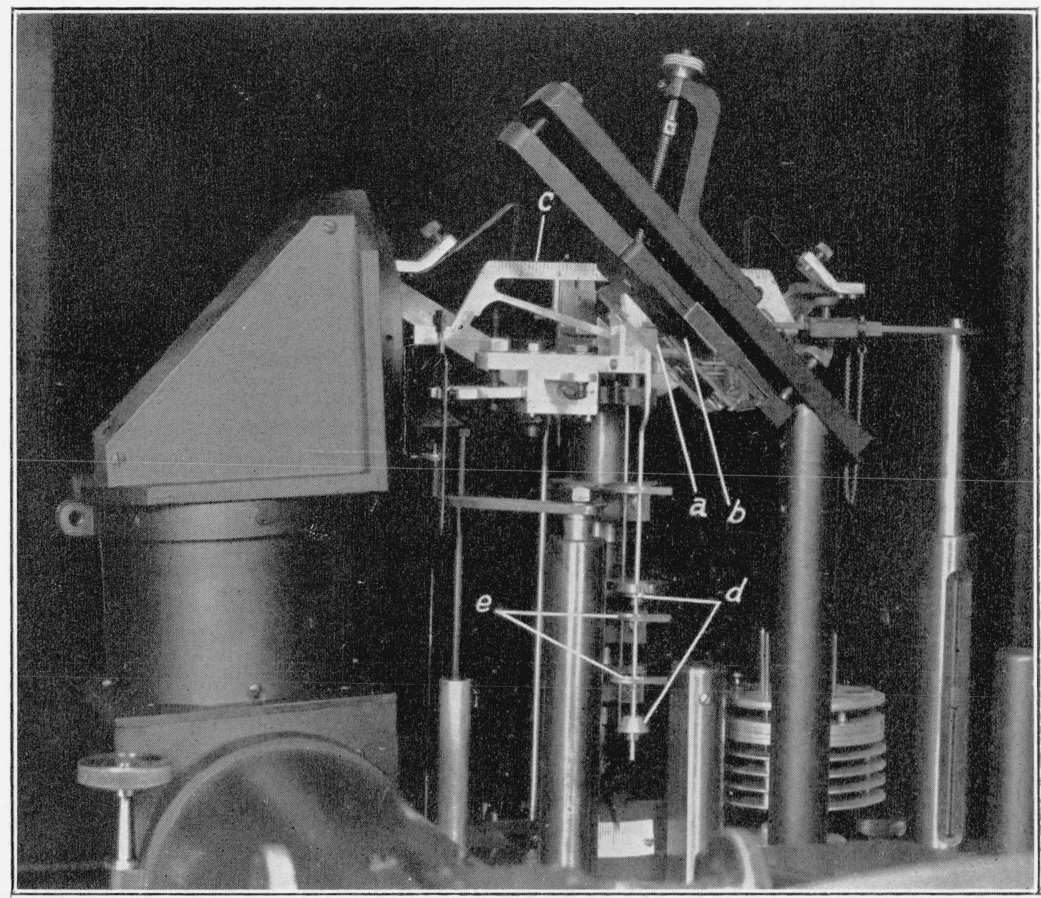

Figure 21.-Balance mechanism from the front.

At the left is the tube which contains lens and prisms for directing the beam of light onto the movable, $a$, and fiducial, $b$, mirrors, which are seen in the center. The balance beam, $c$, is seen behind the mirrors.
The stabilizing arm, with its conical seats, $d$, and the stability weight lifters, $e$, is visible in front of the balance column. 


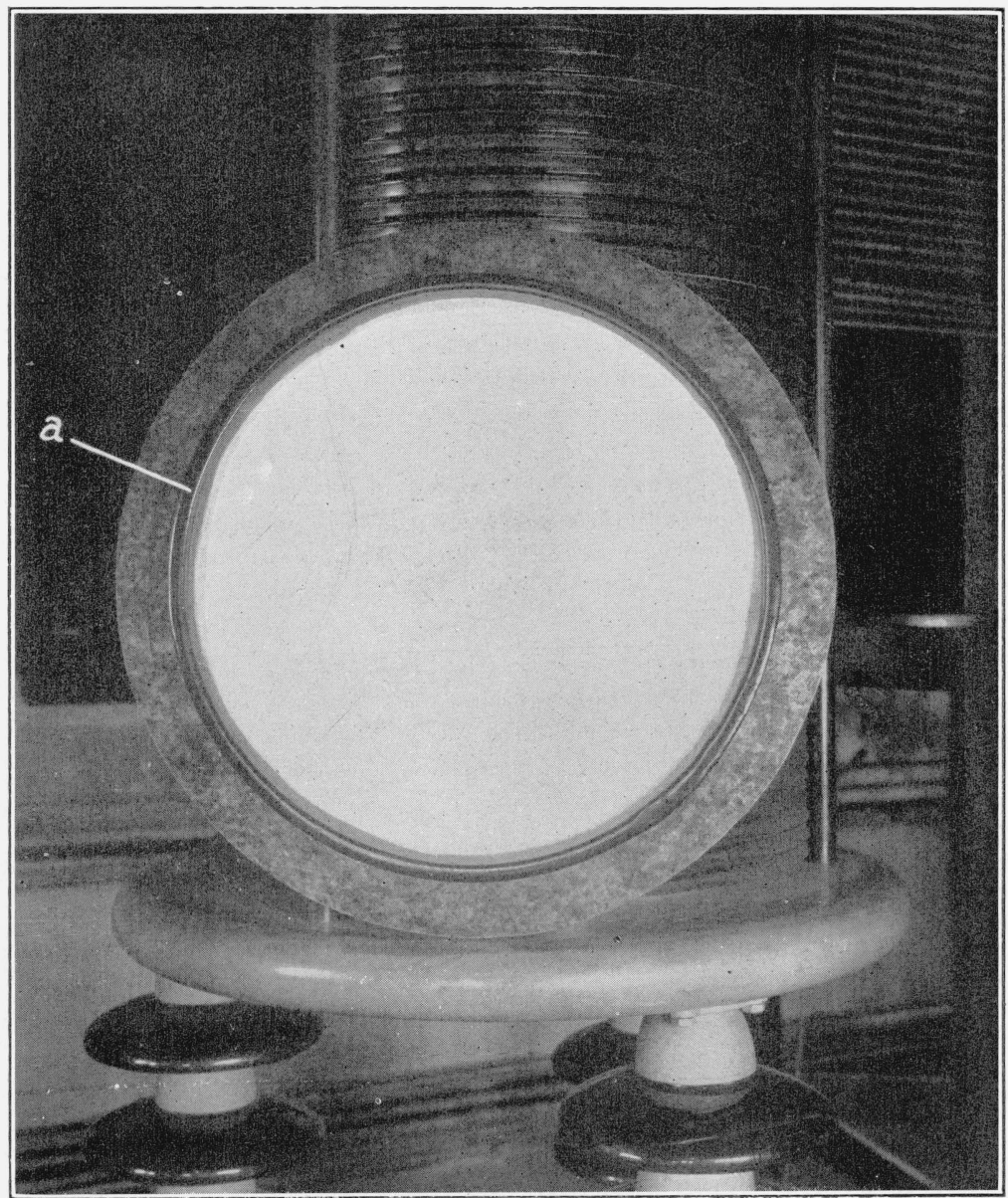

Figure 22.-Capacitor unit.

Micanite disk coated on both sides with tin, showing band of shellac, $a$, around edge of spraved tin electrode. Potential divider itself is seen behind the disk. 
by prisms in the tubular mounting and then strikes both the mirror mounted on the balance beam and the adjacent fiducial mirror. Each of these mirrors reflects its corresponding light beam downward to a prism (not shown in fig. 21 but visible at $d$ in fig. 20) which in turn reflects the two beams back to the horizontal ground-glass scale at the observer's station. The spacing of the lines in the light source is such that each of its images on the screen forms a vernier when observed in connection with the millimeter divisions on the scale. A shift of one coincidence corresponds to $0.05 \mathrm{~mm}$. By means of suitable adjustments of the light source and of the prisms and the scale, the central line of the image from the beam mirror may be brought to the central zero of the scale when the disk and guard ring are very nearly coplanar. The fiducial mirror is then adjusted so that its image also falls at the zero of the scale but lies vertically below and only partly overlaps the image from the beam mirror. The relative position of the two images is thus independent of any movement of the light source or the scale. This use of a fiducial image was described by A. Palm. ${ }^{21}$ The optical magnification of the motion of the disk is approximately 80 .

An aluminum rod extends downward from the center of the balance beam and carries three conical seats at distances of $4.1,8.2$, and 12.3 $\mathrm{cm}$ from the central knife-edge. Weights, ranging from 1 to $10 \mathrm{~g}$, for increasing the stability of the balance may be placed on or removed from one or more of these seats by means of three weight lifters. These lifters are supported and guided by the central column of the balance so that weights can be applied or removed with a minimum disturbance of the balance.

The disk is balanced by a counterpoise carried by the stirrup at the other end of the beam. The counterpoise is of special design in order to avoid change in the equilibrium of the beam with variation in the barometric pressure. The disk, being of an aluminum alloy, has a large buoyancy effect, and if a brass counterpoise (for example) were used the zero position would change if the barometric pressure should change. The obvious remedy is to make the mean density of the counterpoise the same as that of the disk. In addition to this feature, however, it was felt to be expedient to take care of any possible difference in the tendency of metallic surfaces to take up moisture from the air and to give it out. Accordingly, the complex counterpoise shown at $a$ in figure 18 was so constructed that it had the same exposed surface of each kind of metal as well as the same mean density and mass as the parts carried at the other end of the beam.

\section{POTENTIAL DIVIDER AND HOOPS}

The guard hoops and connectors are of thin-wall copper-nickel alloy tubing $8 \mathrm{~mm}$ in diameter, fitted together so as to avoid sharp edges and corners. They rest on maple pegs impregnated with paraffin which are inserted in holes in alternate silica pillars. Normally, the hoops are spaced $2 \mathrm{~cm}$ center-to-center.

The capacitor units, figure 22 , are made of micanite disks $63.5 \mathrm{~cm}$ in diameter and $2 \mathrm{~mm}$ thick. The electrodes consist of coatings of tin, $53 \mathrm{~cm}$ in diameter, sprayed on both sides. To prevent the formation of corona near the edges of the electrodes, melted shellac was

${ }^{21}$ Z. tech. Physik 1, 137-146 (1920). 
extruded to form a band about $3 \mathrm{~mm}$ thick and about $10 \mathrm{~mm}$ wide over the edge of the electrode and adjacent micanite surface. The presence of this band prevents the formation of a visible glow discharge at voltages below about 5,000 volts per unit, which is approximately twice the voltage at which corona would form in the absence of the band of shellac.

The capacitor units are assembled one above another, with aluminum spacers which serve to connect them in series and to hold them $2 \mathrm{~cm}$ apart. The spacers also support the tubular leads which connect each spacer to the appropriate hoop. When the electrometer is used at less than its maximum (110-cm) separation, the unused sections at the bottom of the potential divider are short-circuited. When the hoop spacing used is greater than $2 \mathrm{~cm}$ the appropriate intermediate tap leads and hoops are removed and the corresponding capacitor units are left connected in series between the remaining taps.

The assembly of micanite units and aluminum spacers is clamped together by bolts of insulating material, between a pair of thick aluminum end plates with rounded edges. The complete potential divider is shown at the left in figure 2.

The average capacitance of the units is about $6,500 \mu \mu \mathrm{f}$. Individual values unavoidably varied somewhat from this mean. In view of this fact, units were arranged in such an order ${ }^{22}$ as to obtain the best approximation to the desired condition, in which the total capacitive reactance from the top to any spacer is exactly proportional to the number of units in circuit.

\section{DETAILS OF OPERATION}

\section{WEIGHING}

When everything has been made ready for a voltage measurement, as in a comparison of the electrometer with the "voltmeter," the balance arrestment and the stem clamp are released while the mass $M_{F}$ is on the stirrup and no voltage is applied. The vernier reticle at the lamp is adjusted to bring the image from the fiducial mirror into coincidence with the zero of the scale, the chainomatic control is then adjusted, if necessary, to bring the vernier image from the beam mirror also very close to this zero. Unless the air in the laboratory is unusually quiet, this image will not remain stationary and the "zero reading" is obtained by making 30 independent readings at equal time intervals (usually every 3 seconds) and taking the mean.

The stem is then clamped, the mass $M_{F}$ removed, and voltage is applied and adjusted to approximately the selected value. The stem clamp is then released. Because of the decreased stability of the balance the motion is now somewhat slower, and the amplitude is greater both by reason of the greater sensitivity and because of fluctuations in the applied voltage. By suitable manipulation of the applied voltage the spot of light can be kept very close to the fiducial mark for intervals of several seconds. When 10 satisfactory readings have been obtained in which both instruments have been reasonably steady for an interval as great as the period of the electrometer, the stem is clamped, the voltage removed, the mass $M_{F}$ replaced, and the stem again released. A second set of 30 readings

?2 W. A. Price. The Measurement of Electrical Resistance, Oxford, Clarendon Press, p. 83-84 (1894). 
to obtain the balance zero is then made. If the zero readings differ only slightly, their average is taken as $S_{0}$ and the reading at which the settings were made with voltage applied is taken as $S_{v}$. It is then assumed that the respective air forces $A_{0}$ and $A_{0}$ are equal and drop out of the final result. Just before and just after each group of weighings the barometer and the wet- and dry-bulb thermometers are read to give data needed for the calculation of the dielectric constant of the air. Temperatures are read from six other thermometers. Two of these are located in holes in the upper plate and the lower plate, respectively, one is clamped against one of the silica pillars, and the remaining three are clamped against the three invar legs which support the lower plate. The temperature of the upper plate is used in computing the radius of the disk, and the temperatures of the lower plate and of the invar legs are used in computing the separation from the previously determined values which for convenience had been reduced to a conventional standard temperature of $20^{\circ} \mathrm{C}$.

To minimize errors arising from air forces it was found essential to maintain a thermostatic control of the air temperature. The indoor temperature was controlled at a value equal to the estimated maximum outdoor temperature. This is usually nearly constant for a period from $1 \mathrm{p}$. m. to $4 \mathrm{p}$. $\mathrm{m}$. each day and hence during this time the indoor and outdoor temperatures are nearly equal and air currents resulting from temperature differences are a minimum. Also the air in the dome, having already come to thermal equilibrium with the air in the laboratory, shows no tendency to produce a steady air force on the disk by expansion or contraction. The air in the laboratory is stirred continuously except during each weighing and for an interval of about 10 minutes before the weighing is begun.

The thermostatic control and the stirring also serve to minimize errors in potential distribution among the hoops, which might result from the relative changes in capacitance of the end and the central sections of the potential divider, occasioned by the different rates at which these sections follow any change in room temperature.

\section{MEASUREMENT OF COPLANARITY}

The determinations of the height $h_{m}$ of the disk relative to its guard ring are made at least twice daily, just before and just after each set of weighings. For this purpose there were constructed two special "coplanarity" microscopes. The essential features of one of these microscopes are shown in figure 27. Each consists of a 16-mm objective and a $12 \times$ eyepiece held about $70 \mathrm{~cm}$ apart. The eyepiece contains a ruled reticle which is used in measuring the width of the gap. Each objective was modified by the insertion of a right-angled prism between its lenses so that the central ray coming vertically downward from the disk through the front lens of the objective is reflected by the prism to pass horizontally through the back lens, intermediate lens and eyepiece. An intermediate lens (8-diopter) between the eyepiece and the objective can be moved through a range of $35 \mathrm{~mm}$ and serves to give a fine adjustment of focus and thus a measurement of object distance (over a range of $0.14 \mathrm{~mm}$ ). The intermediate lens is mounted on a rack, the pinion of which is supported on the microscope tube and carries an indexed head and revolution counter to 
show the position of the lens. Calibration curves for each indexed head and counter in terms of change in object distance were obtained experimentally by using a Zeiss Optimeter. A change of $1 \mu$ in object distance corresponds to about $1 \mathrm{~mm}$ on the micrometer head.

The two microscopes rest in a channel in an aluminum mounting. One-half of this is shown in longitudinal section at (29), figure 27 . When in place this mounting extends completely through the instrument and can be clamped against the lower surface of the guard ring along any desired diameter. Two clamps, hinged near the ends of the channel, bear against the upper side of the guard ring, while two pairs of projections $20 \mathrm{~cm}$ from the ends bear against the lower side of the guard ring. Tightening the clamp screws bends the channel and moves the (inner) objective ends of the microscopes downward. This bending action is utilized to bring the microscopes within their focusing range. To prevent any change in this bending as a result of differential thermal expansion or of slipping at the points of contact after clamping, because of strain then set up, one pair of projections is mounted on two flexible strips which are mounted vertically to resist vertical thrust but permit the relief of horizontal strain. The other two projections are formed integral with the sides of the channel.

Each microscope can be moved horizontally in or out with respect to the channel to enable the observer to view either the disk or the guard ring at will. The outer end of the microscope tube at (29) is guided by four steel balls which limit shake and rotation but permit longitudinal motion. The inner end is guided by a groove accurately milled and scraped in the back of the objective housing. This groove rests on the top of an adjustable steel finger (30) mounted in the aluminum channel. By making observations on the almost optically flat surface of the stainless-steel insert, corrections were obtained for the slight vertical motion of the objectives resulting from unavoidable irregularities in their grooves. The magnification of the microscope is about 200 diameters.

Before the microscopes are used the position of the disk is made definite in the following manner. The mass $M_{P}$ is removed from the stirrup and the stem clamp is released in the normal manner. The counterpoise end of the beam being heavier tilts down until it rests lightly against a small projection, carried on the chainomatic plunger. The observer at the scale then operates the chainomatic control to raise the counterpoise end of the beam until the vernier image from the beam mirror has the desired reading $S_{m}$. This is usually chosen to be in coincidence with the image reflected from the fiducial mirror. Under these conditions the disk hangs freely from its knife-edge in the same way as when voltage is being measured, and the stresses in the beam and at the knife edges differ from their normal values by only the small amount caused by a change $M_{R} g$ in the load.

Determinations are made at six equally spaced azimuths. Each determination is computed from 10 independent settings for sharpest focus on the disk near its edge and from 10 similar settings on the bushing. Each set of 10 readings is averaged and the vertical height in centimeters (from an arbitrary zero) corresponding to this average, is read off from the calibration curve of the microscope. The difference between these heights for each pair of sets is corrected for irregularities in the groove which guides the microscope objective and the corrected result is taken as the difference in height between the edge 
of the disk and the guard ring at that azimuth. The six differences thus obtained are then averaged. The entire operation requires about 40 minutes.

\section{GAGING}

The gaging of the distance between the guard ring and the lower plate, both for the purpose of adjusting their opposed surfaces to parallelism and for accurately determining their separation $c$, is done as shown in figure 27. The long gage rod (33) rests on the segmental contact block (34) and protrudes through the gaging hole in the guard ring. A short gage block (6) is placed beside the rod and the difference in level of their upper ends is measured by the surface gage (5), which reads to 0.0001 inch. To protect the lower plate from damage it was covered during gaging by an aluminum plate, which had openings to receive and locate the segmental block (34) in the correct position. The separation $c$ is computed from the readings of the dial indicator, the known thickness of the bushing, and the lengths of the blocks and the rod. The six thermometers attached to the instrument are read at the time of gaging to permit the result to be reduced to the standard $20^{\circ} \mathrm{C}$ basis.

The gage rods and blocks were made of nickel-iron alloy which had an expansivity of $2 \times 10^{-6}$ per ${ }^{\circ} \mathrm{C}$. Their ends were plated with chromium to minimize wear. The shorter ones were lapped to be correct to $2 \mu$ and the longer to 0.001 percent. It was found much less expensive to make two complete sets of gages rather than a single set because the precise adjustment was thereby greatly facilitated. Each set comprises 15 rods, $1.9 \mathrm{~cm}$ in diameter and of lengths $12,14,16,18,20$, $30,40 \ldots 120 \mathrm{~cm}$; and 10 blocks, $3.2 \mathrm{~cm}$ in diameter and of length ranging from 4.8845 to $12.8845 \mathrm{~cm}$ by 2 -cm intervals. These latter unusual lengths combined with the particular thickness of the guard ring facilitated the gaging.

\section{MEASUREMENT OF DIAMETERS}

The diameters of the duralumin disks and also of the apertures in the steel bushings are measured by comparing them with the lengths of a set of duralumin line standards $10,12,14$, and $16 \mathrm{~cm}$ between lines. The measurements were made with two microscopes having micrometer eyepieces, for which one division of the indexed head of the screw corresponds to about $1 \mu$.

When a disk is to be measured, the microscopes are clamped firmly to a heavy steel bar which is supported above a turntable. The axes of the microscopes are vertical and at such a distance apart that the marks at the ends of the line standard are near the centers of their fields of view, when the standard lies along a diameter of the turntable. The focusing of the microscopes, after an initial adjustment, is done by vertical adjustment of the bar.

When the diameter of the aperture in a bushing is to be measured, the microscopes are mounted on studs which rise from a heavy castiron ring $30 \mathrm{~cm}$ in internal diameter, which is supported directly from the inverted bushing by three small brass levelling screws and is guided in its rotation by the external cylindrical surface of the bushing. The focusing of the microscopes after they are once aligned is done by the levelling screws which support the ring. In both cases measurements are made along 24 equally spaced diameters. 
These diameter measurements involve in effect the notoriously difficult comparison of line standards with end standards. However, readings made under widely different types of illumination were so consistent as to indicate that the errors were probably well below the tolerance of $8 \mu$ which corresponds to 0.01 percent in voltage. The fact that the ultimate object of the measurements is to determine the mean of the diameters of a solid disk and of an aperture doubtless tends to compensate the systematic error.

To reduce the results to the diameters at the standard temperature, $20^{\circ} \mathrm{C}$, at which the length of the line standard is known, the values for the bushing aperture must be corrected for thermal expansion, using the difference in expansivity of stainless steel and duralumin. No correction is needed for the disks since their expansivity is the same as that of the standard.

\section{SURVEY OF THE DISK}

To obtain the data needed to locate the average height of the rim of the disk with respect to the six particular points viewed in the coplanarity microscopes, and also to determine the general shape of its lower surface and the corresponding value of $h_{d}$, it is necessary to make a precise survey of this surface.

Because of the softness of the aluminum alloy, it was not found feasible to produce a sufficiently high polish on the surface of the disk to permit the use of interference methods for this purpose. The survey is therefore made by reading an indicator, the contact finger of which is placed in succession at about 120 points uniformly distributed over the disk. The difference between the mean of these readings and the mean of 120 other readings made at points which lie along a circumference close to the edge, indicated by line $Y Y$, figure 4, is denoted by $h_{d}$ and is used as indicated in the formulas. A graph of the readings at the edge, plotted against azimuth, serves to correlate the mean height of the rim with the height of the particular six points of the circumferential set at which observations are made with the coplanarity microscopes.

It was found that the pressures exerted by the contact fingers of the dial indicators available were excessive and would cause scratching of the disk. An optical indicator was therefore constructed embodying the principles of the Tuckerman strain gage. ${ }^{23}$ In this indicator the motion of a contact finger, held against the disk by a very light force, tilts a prism, the angular motion of which, relative to a fiducial roof prism, is observed with an autocollimator. One division on the autocollimator scale corresponds to $0.0005 \mathrm{~mm}$ at the disk surface.

As an aid in surveying the disks and also in adjusting the stems and measuring the diameters of the disks, as described in the preceding section, a small precision lathe was mounted on a wall with its headstock down and its axis vertical. A stepped faceplate carried on the spindle of the headstock thus forms a turntable on which the disk can be laid, inverted, with its stem extending down through the hollow spindle. A bracket rigidly fastened to the lathe supports a surface plate on which the block carrying the optical indicator mentioned above is placed.

In carrying out the survey the effect of any tilt of the surface of the disk with respect to a plane at right angles to the axis of the lathe

${ }^{23}$ Proc. Am. Soc. Testing Materials 23, pt. 2, 602-610 (1923). 
is eliminated by taking two sets of readings, the disk being rotated $180^{\circ}$ between them. Irregularities and bending of the surface plate on which the indicator block slides are allowed for by suitable corrections. These are obtained by substituting an optical flat for the electrometer disk and making a pair of diametrical traverses across it. When the disk is hanging in the electrometer its shape will differ slightly from that which it assumes when it is supported near its rim upside down on the stepped faceplate. The magnitude of this change of shape, as calculated from observations of the deflection produced at the center of the disk when a known weight was hung from the stem, was found to be entirely negligible.

\section{MEASUREMENT OF HOOP-POTENTIAL DISTRIBUTION}

The ratio of the capacitive reactances above and below each of the tap points in the potential divider is measured by means of the bridge circuit shown at the left in figure 23. The two arms $R_{1}$ and $R_{2}$ are noninductive resistances; the other two arms are formed by the total

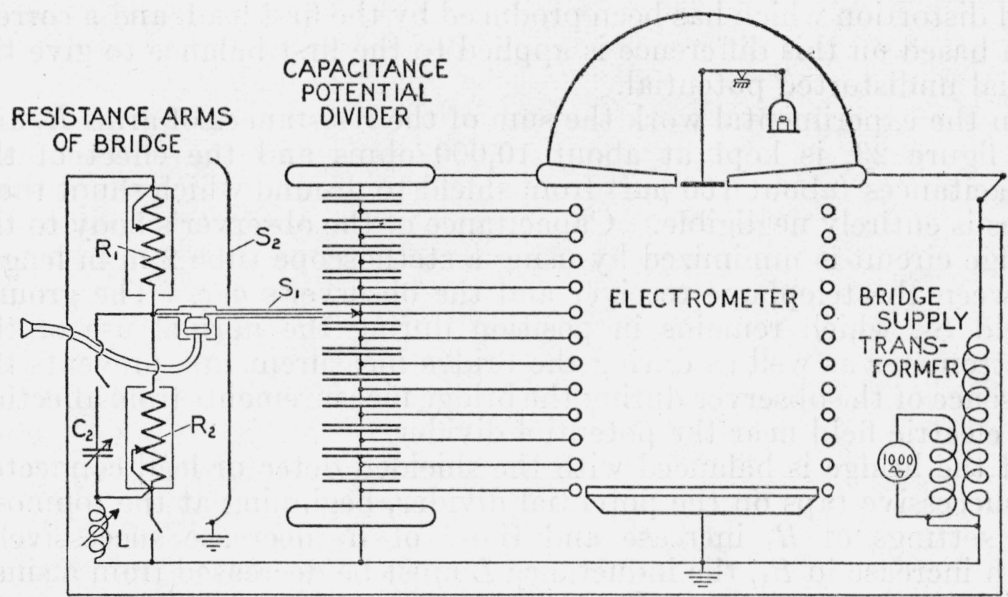

FIGURE 23.-Schematic diagram of audio-frequency bridge used to measure potential distribution.

capacitances effectively present between the tap under test and the electrometer plates. The bridge is supplied with 1,000-cycle current at about 350 volts.

As far as possible, during these bridge measurements, all leads and connected apparatus are located and connected as in the normal use of the electrometer, and thus introduce no disturbing capacitance. The connection from the spacer at the tap point to the telephone receiver, which constitutes the bridge detector, is, however, liable to distort the electric field and requires special consideration. This lead can be shielded, as is indicated in figure 23, up to a point a few millimeters from its junction with the spacer in the potential divider. If this shield, $S_{1}$ is connected to the junction of the two resistance arms of the bridge, no difference of potential will exist across the 
capacitance between the shield and the lead within it; and the capacitances between the shield and external conductors will act merely as shunts around the resistance arms of the bridge, where they affect only the quadrature component of the impedances of these arms.

The mere presence of the shielded lead will, however, produce a distortion of the electric field and a consequent change in potential of the hoops, unless the lead should lie exactly in that surface which, prior to the insertion of the lead, was the equipotential which contained the spacer under test. By suitably mounting the telephone receiver, a considerable length of the lead at the end near the potential divider can be made to lie fairly close to this equipotential surface; and its departure from this ideal position at greater distances will only slightly distort the field. As a further precaution, however, it was found desirable to use two such shielded leads, symmetrically placed and electrically in parallel, but physically located at different azimuths so as to have but little mutual effect. The change in the bridge balance produced when the second lead is added is taken as a measure of the field distortion which has been produced by the first lead, and a correction based on this difference is applied to the first balance to give the initial undistorted potential.

In the experimental work the sum of the resistances of arms $R_{1}$ and $R_{2}$, figure 23 , is kept at about $10,000 \mathrm{ohms}$ and the effect of the capacitances (about $100 \mu \mu \mathrm{f}$ ) from shield to ground which shunt these arms is entirely negligible. Capacitance of the observer's body to the bridge circuit is minimized by using a stethoscope tube $2 \mathrm{~m}$ in length between the telephone receiver and the observer's ear. The ground shield $S_{2}$, which remains in position during the normal use of the electrometer as well as during the bridge measurements, prevents the presence of the observer during the bridge measurements from affecting the electric field near the potential divider.

If the bridge is balanced with the shielded detector lead connected to successive taps on the potential divider, beginning at the topmost, the settings of $R_{1}$ increase and those of $R_{2}$ decrease successively. With increase in $R_{1}$, the inductance $L$ must be decreased from a small positive value until when $R_{1}$ is large, the capacitance $C_{2}$ must be connected in parallel with $R_{2}$. This is consistent with the effect to be expected from the capacitance between the shield $S_{1}$ and the divider. The slight shunting effect of this capacitance on the effective ratio of the resistances is therefore computed on the assumption that the voltages to ground of all the hoops are in the same phase, and that the small observed phase shifts are due only to the shield capacitance. Under normal conditions this shunting correction affects $G^{\prime}{ }_{k}$ by only 10 parts in a million. The correction to the voltage deduced from the change in balance when the second shielded lead is attached is only about 0.03 percent, when the separation is $48 \mathrm{~cm}$, but is about 0.25 percent when the separation is $110 \mathrm{~cm}$.

\section{MATHEMATICAL DETAILS}

\section{CORRECTION FOR NONUNIFORMITY OF HOOP-POTENTIAL DISTRIBUTION}

The electric potential $V(r, y)$ at any point within the space bounded by the plane, parallel electrometer plates at a separation $b$ and by the 
right cylinder of radius $R$ tangent internally to the set of guard hoops, must satisfy Laplace's equation, which in cylindrical coordinates is

$$
\frac{\partial^{2} V}{\partial r^{2}}+\frac{1}{r} \frac{\partial V}{\partial r}+\frac{\partial^{2} V}{\partial y^{2}}+\frac{1}{r^{2}} \frac{\partial^{2} V}{\partial \theta^{2}}=0
$$

From the symmetry of the apparatus it is evident that $\frac{\partial^{2} V}{\partial \theta^{2}}=0$. The problem is, therefore, to find $V$ as a function of $r$ and $y$ which satisfies eq 18 and also meets the following boundary conditions (which assume the disk to be coplanar with the guard plate and the gap to be zero):

$$
\left.\begin{array}{l}
\text { For } y=0, \quad V=0 \text { for all values of } r \\
\text { For } y=b, V=V_{S} \text { for all values of } r \\
\text { For } r=R, V=V_{R}(y)
\end{array}\right\}
$$

where $V_{R}(y)$ describes the distribution of potential at the cylindrical boundary. This function can be expressed in the form

$$
V_{R}(y)=V_{S} \frac{y}{b}+V_{S} \sum_{k=1}^{k=\infty} G_{k} \sin \frac{k \pi y}{b}
$$

Here the first term corresponds to the ideal linear variation of potential, while the second term is the Fourier's series describing the departure from this ideal, $k$ being an integer.

In searching for the solution let us assume that it has the form

$$
V=V_{S} \frac{y}{b}+F_{1}(y) F_{2}(r)
$$

Making the necessary differentiations and substituting the results in eq 18 gives

$$
F_{1}(y) \frac{\partial^{2} F_{2}(r)}{\partial r^{2}}+\frac{F_{1}(y)}{r} \frac{\partial F_{2}(r)}{\partial r}+F_{2}(r) \frac{\partial^{2} F_{1}(y)}{\partial y^{2}}=0,
$$

or on rearranging terms to separate the variables

$$
\frac{1}{F_{2}(r)} \times \frac{\partial^{2} F_{2}(r)}{\partial r^{2}}+\frac{1}{r F_{2}(r)} \times \frac{\partial F_{2}(r)}{\partial r}=-\frac{1}{F_{1}(y)} \times \frac{\partial^{2} F_{1}(y)}{\partial y^{2}}
$$

Since the left-hand member of eq 23 is a function of $r$ only, while the right-hand member is a function of $y$ only, each member must be equal to a constant. Let this constant be designated by $\mu^{2}$.

The equation involving $r$ is then

$$
\frac{\partial^{2} F_{2}(r)}{\partial r^{2}}+\frac{1}{r} \frac{\partial F_{2}(r)}{\partial r}-\mu^{2} F_{2}(r)=0
$$

a solution of which is

$$
F_{2}(r)=J_{0}(i \mu r),
$$

where $J_{0}$ is Bessel's function with parameter zero. 
The equation involving $y$ is

$$
\frac{\partial^{2} F_{1}(y)}{\partial y^{2}}+\mu^{2} F_{1}(y)=0
$$

the solution of which can be written

$$
F_{1}(y)=V_{s} A \cos \mu y+V_{s} B \sin \mu y
$$

Since $\mu$ is entirely arbitrary the complete solution satisfying eq 18 is found by adding all possible solutions of the above forms and is

$$
V=\frac{V_{s} y}{b}+V_{s} \sum_{k=1}^{k=\infty}\left\{A_{k} \cos \left(\mu_{k} y\right)+B_{k} \sin \left(\mu_{k} y\right)\right\} J_{0}\left(i \mu_{k} r\right)
$$

To fit the boundary conditions given by eq 19, the series must vanish at $y=0$ and $y=b$ for any value of $r$. Hence the factor multiplying $J_{0}\left(i \mu_{k} r\right)$ must vanish for any value of $k$ for these particular values of $y$. Hence

$$
A_{k}=0 \text { and } \mu_{k} b=k \pi
$$

Inserting these relations in eq 28 gives

$$
V=\frac{V_{s} y}{b}+V_{s} \sum_{k=1}^{k=\infty} B_{k} \sin \left(\frac{k \pi y}{b}\right) \times J_{0}\left(i \frac{k \pi r}{b}\right)
$$

At the particular radius $R$ this becomes

$$
V_{R}=\frac{V_{s} y}{b}+V_{s} \sum_{k=1}^{k=\infty} B_{k} \sin \left(\frac{k \pi y}{b}\right) \times J_{0}\left(i \frac{k \pi R}{b}\right)
$$

A comparison of eq 31 with eq 20 indicates that the boundary condition fixed by the latter will be satisfied if

$$
B_{k}=\frac{G_{k}}{J_{0}\left(\frac{i k \pi R}{b}\right)}
$$

and the final solution for the potential anywhere within the hoopenclosed space becomes

$$
V=\frac{V_{s} y}{b}+V_{s} \sum_{k=1}^{k=\infty} G_{k} \sin \left(\frac{k \pi y}{b}\right) \times \frac{J_{0}\left(\frac{i k \pi r}{b}\right)}{J_{0}\left(\frac{i k \pi R}{b}\right)}
$$

To calculate the attractive force produced by such an electric field we have for the vertical component of potential gradient at any point 


$$
\frac{\partial V}{\partial y}=\frac{V_{s}}{b}\left[1+\sum_{k=1}^{k=\infty} k \pi G_{k} \cos \left(\frac{k \pi y}{b}\right) \times \frac{J_{0}\left(\frac{i k \pi r}{b}\right)}{J_{0}\left(\frac{i k \pi R}{b}\right)}\right]
$$

The surface density of charge $\sigma$ on the upper plate is related to the potential gradient adjacent to the surface by the equation

$$
\sigma=\frac{1}{4 \pi}\left(\frac{\partial V}{\partial y}\right)_{y=b}
$$

Combining this relation with eq 34 gives for the charge density on the disk

$$
\sigma=\frac{V_{s}}{4 \pi b}\left[1+\sum_{k=1}^{k=\infty}(-1)^{k} k \pi G_{k} \frac{J_{0}\left(\frac{i k \pi r}{b}\right) \mid}{J_{0}\left(\frac{i k \pi R}{b}\right)}\right]
$$

For the present applications the summation corresponds to the relatively small departure from the ideal distribution and is small compared with unity. Neglecting the square of this summation therefore, we may write

$$
\sigma^{2}=\frac{V_{s}^{2}}{16 \pi^{2} b^{2}}\left[1+2 \sum_{k=1}^{k=\infty}(-1)^{k} k \pi G_{k} \frac{J_{0}\left(\frac{i k \pi r}{b}\right)}{J_{0}\left(\frac{i k \pi R}{b}\right)}\right]
$$

The attractive force is given by

$$
F=2 \pi \int_{0}^{r d} \sigma^{2} \times 2 \pi r d r
$$

Substituting eq 37 in eq 38 , noting that

$$
\int_{0}^{x} x J_{0}(x) d x=x J_{1}(x)
$$

where $J_{1}$ is Bessel's function with parameter 1 , gives

$$
F=\frac{V_{s}^{2}}{4 b^{2}}\left[\frac{r_{d}^{2}}{2}+2 \sum_{k=1}^{k=\infty}(-1)^{k} \frac{b r_{d} G_{k} J_{1}\left(\frac{i k \pi r_{d}}{b}\right)}{i J_{0}\left(\frac{i k \pi R}{b}\right)}\right]
$$

or on rearranging

$$
F=\frac{V_{s}^{2} r_{d}^{2}}{8 b^{2}}\left[1+4 \sum_{k=1}^{k=\infty}(-1)^{k} \frac{b}{i r_{d}} G_{k} \frac{J_{1}\left(\frac{i k \pi r_{d}}{b}\right)}{J_{0}\left(\frac{i k \pi R}{b}\right)}\right]
$$

and on solving for $V_{s}$, again neglecting higher powers of the summation, 


$$
V_{s}=\frac{2 b \sqrt{2 F}}{r_{d}}\left[1-\sum_{k=1}^{k=\infty}(-1)^{k} k \pi G_{k} \frac{2 J_{1}\left(\frac{i k \pi r_{d}}{b}\right)}{\frac{i k \pi r_{d}}{b}} \times \frac{1}{J_{0}\left(\frac{i k \pi R}{b}\right)}\right]
$$

which is in the form

$$
V_{s}=\frac{2 b \sqrt{2 F}}{r_{d}}\left[1-\sum_{k=1}^{k=\infty} C_{k} G_{k}\right]
$$

where

$$
C_{k}=(-1)^{k} \pi k \cdot \frac{2 J_{1}\left(\frac{i k \pi r_{d}}{b}\right)}{\frac{i k \pi r_{d}}{b} \times J_{0}\left(\frac{i k \pi R}{b}\right)}
$$

In cases where the terms neglected in passing from eq 36 to eq 37 are appreciable, the voltage is given rigorously by

$$
V_{s}=\frac{2 b \sqrt{2 F}}{r_{d}}\left[1+2 \sum_{k=1}^{k=\infty} C_{k} G_{k}+\Delta_{2}\right]^{-\frac{1}{2}},
$$

where

$$
\begin{aligned}
& \Delta_{2}=\sum_{k=1}^{k=\infty} \frac{\pi^{2} k^{2}\left[\left\{J_{0}\left(\frac{i k \pi r_{d}}{b}\right)\right\}^{2}+\left\{J_{1}\left(\frac{i k \pi r_{d}}{b}\right)\right\}^{2}\right]}{\left\{J_{0}\left(\frac{i k \pi R}{b}\right)\right\}^{2}} G_{k}^{2}+\left\{\sum_{\substack { k=1 \\
\begin{subarray}{c}{l=1 \\
l \neq k{ k = 1 \\
\begin{subarray} { c } { l = 1 \\
l \neq k } }\end{subarray}}^{k=\infty}\right. \\
& \left.\frac{(-1)^{k+l} 2 k l \pi^{2} b\left[k i J_{1}\left(\frac{i k \pi r_{d}}{b}\right) \times J_{0}\left(\frac{i l \pi r_{d}}{b}\right)-l J_{0}\left(\frac{i k \pi r_{d}}{b}\right) \times i J_{1}\left(\frac{i l \pi r_{d}}{b}\right)\right]}{\pi r_{d}\left(l^{2}-k^{2}\right) J_{0}\left(\frac{i k \pi R}{b}\right) J_{0}\left(\frac{i l \pi R}{b}\right)} G_{k} G_{\imath}\right\}
\end{aligned}
$$

It may be noted that for large values of the arguments, such as correspond to large values of $k$, the semiconvergent expansions for the Bessel's functions can be used to express $C_{k}$ in the form

$$
C_{k}=(-1)^{k} \frac{2 b}{r_{d}}\left(\frac{R}{r}\right)^{\frac{3}{2}} \frac{\left(1+\frac{b}{8 k \pi r_{d}} \cdots\right)}{\left(1-\frac{3 b}{8 k \pi R} \cdots\right)} e^{k \pi\left(r_{d}-R\right) / b}
$$

Since $R$ is considerably larger than $r_{d}$ the exponential factor in this expression becomes exceedingly small for very moderate values of $k$. In particular, the ripple ${ }^{24}$ in potential which results from the finite (2-cm) spacing of the hoops and which introduces terms of order $k=b$, will be affected by the factor $e^{\pi\left(r_{d}-R\right)}$, which for $R=50 \mathrm{~cm}$ and

${ }_{24}$ This ripple has been treated by Snow (see footnote 7) from a somewhat different approach with similar results. 
$r_{d}=8 \mathrm{~cm}$ is about $10^{-50}$. However, when $b$ is sufficiently large compared with $\pi R$ and $\pi r_{d}$, the values of $C_{k}$ increase with successively increasing values of $k$, so that the summation will converge only if the successive values of $G_{k}$ decrease at a sufficiently rapid rate.

\section{SOURCES OF ERROR IN FOURIER COEFFICIENTS, $G_{k}{ }^{\prime}$}

Although the experiments reported in table 5 indicate that the values of $C_{k}$ are correct and that changes in potential distribution are adequately allowed for by the $\Sigma C_{k} G_{k}{ }^{\prime}$ correction term, there remains a further possible source of error if the values of $G_{k}^{\prime}$ computed from eq 17 differ from the true values of $G_{k}$, as defined by eq 14 . The difference between these values may be written

$$
\begin{aligned}
G_{k}^{\prime}-G_{k} & =\frac{2}{b}\left[\sum_{m=1}^{m=n}\left\{s\left(\frac{V_{m}}{V_{s}}-\frac{m}{n+1}\right) \sin \frac{k \pi m}{n+1}-\int_{(m-1 / 2) s}^{(m+1 / 2) s}\left(\frac{V_{R}(y)}{V_{s}}-\frac{y}{b}\right) \sin \frac{k \pi y}{b} d y\right\}\right. \\
& \left.-\int_{0}^{s / 2}\left(\frac{V_{R}(y)}{V_{s}}-\frac{y}{b}\right) \sin \frac{k \pi y}{b} d y-\int_{b-s / 2}^{b}\left(\frac{V_{R}(y)}{V_{s}}-\frac{y}{b}\right) \sin \frac{k \pi y}{b} d y\right]
\end{aligned}
$$

where $s$ is the spacing of the hoops, center to center. The significance of this expression can be seen best perhaps by the graphic interpretation shown in figure 24. Here in the upper-left corner is shown an assortment of assumed potential distribution curves similar to figure 12 and corresponding to different alternative hypothetical cases.

Line $A$ illustrates the ideal linear potential distribution, curve $B$ a smooth distribution such as would result if only the first few coefficients $G_{k}$ had appreciable values. Curves $C$ and $D$ show other distributions in which the potential at points in the spaces between the hoops departs from the smooth gradient of curve $B$. To show the relations involved more clearly, the difference between each curve and the line $A$, i. e., the quantity $\frac{V_{R}(y)}{V_{S}}-\frac{y}{b}$, is plotted to a much enlarged scale in the central part of the figure. The end section $I$ of curve $B$, containing the first three hoops, is shown at $I B^{\prime}$, and a typical intermediate section $I I$ spanning two hoops and half of each space is shown magnified at $I I B^{\prime}, I I C^{\prime}$, and $I I D^{\prime}$ for the three types of distribution $B, C$, and $D$, respectively.

At $S_{I}$ and $S_{I I}$ are shown (ordinate scale at the right) portions of a graph of the factor $\sin k \pi y / b$ (for the case $k=1$ ) ${ }^{25}$ corresponding to sections $I$ and $I I$, respectively.

At the bottom of the figure $I B^{\prime \prime}, I I B^{\prime \prime}, I I C^{\prime \prime}$, and $I I D^{\prime \prime}$, respectively, are shown portions of the corresponding graphs of the resultant

quantity $\left(\frac{V_{R}(y)}{V_{S}}-\frac{y}{b}\right) \sin \frac{k \pi y}{b},(k=1)$ which occurs in eq 46 .

Now at the points $P_{1}, P_{2} \ldots P_{m}$, where $y=\frac{1}{n+1}, \frac{2}{n+1}, \ldots \frac{m}{n+1}$,

${ }^{25}$ For convenience in drawing, the negative of this factor (i. e., $-\sin \pi y / b$ ) has been plotted in figure 24 . 

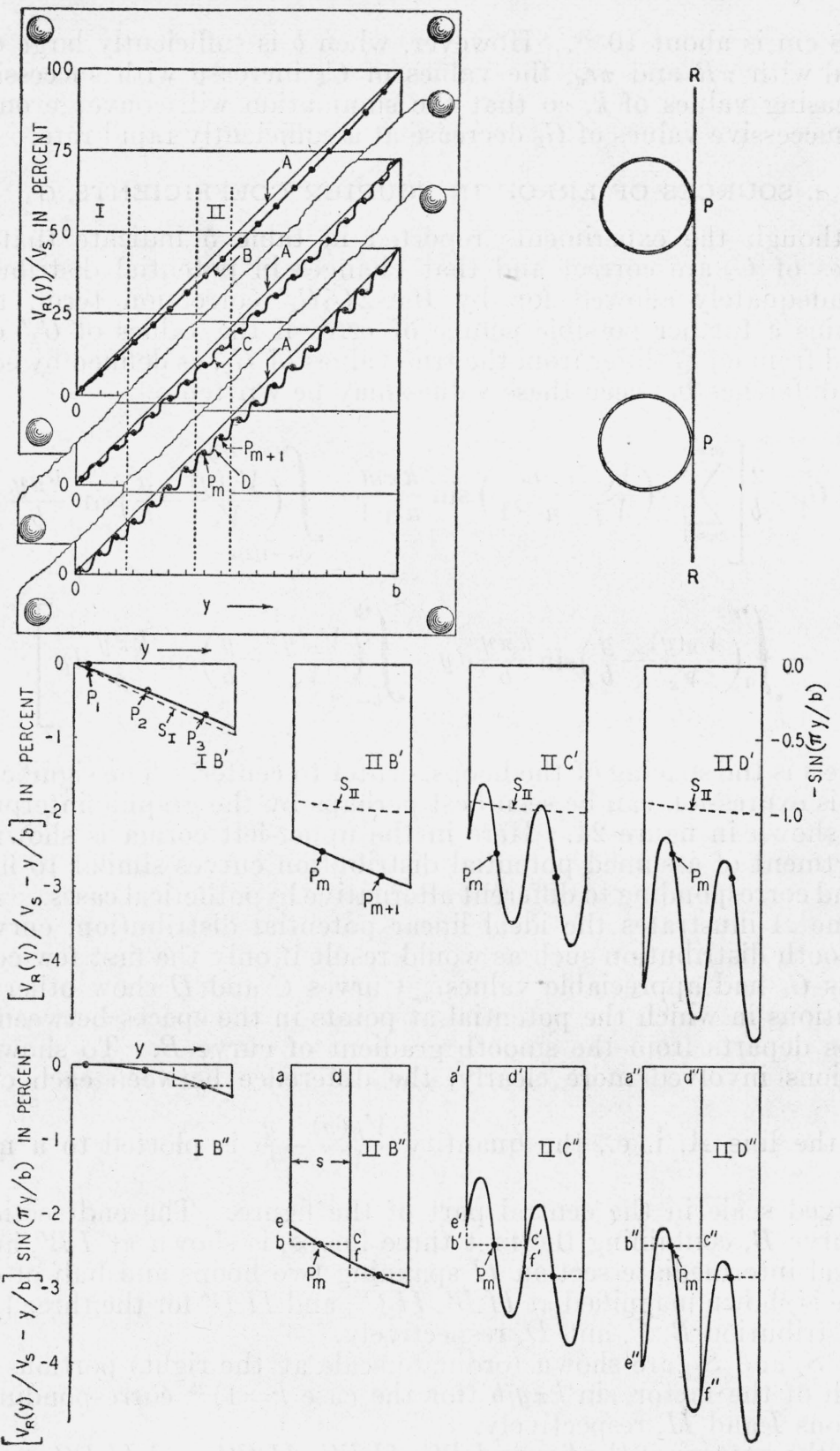

Figure 24.-Graphic illustration of origin of error in Fourier coefficients $G_{k}{ }^{\prime}$.

At $A, B, C$, and $D$ are shown examples of the four following types of potential-distribution curves: $A$, the ideal linear distribution; $B$, a smooth curve similar to the resultant of the first few terms of the Fourier series, and passing nearly through the points $P_{1}, P_{2}, \ldots P_{m}$, which were observed with 6 -cm spacing and 110-cm separation; $C$, a curve having a ripple of such magnitude as to satisfy the requirement that the potential be independent of height at points close to each hoop and $D$, a curve having a ripple of the shape that is produced if the potential gradient has a radial as well as an axial component, and approximately of the magnitude present with a plate separation of $110 \mathrm{~cm}$ and the abnormal hoop spacing of $6 \mathrm{~cm}$. Below are shown to a magnified scale for section $I$ of curve $B$ and section $I I$ of curves $C$ and $D$, the departure $\left(V_{B}(y) / V_{\mathcal{S}}-y / b\right)$ from curve $A$. At the bottom are shown the product of the departure multiplied by the factor $\sin \pi y / b$ for the three cases, this product being the quantity which appears in eq 46 which gives the difference between the true coefficients $G_{k}$ and the approximate values $G_{k}^{\prime}$. 
respectively, $V_{R}(y)$ has the values $V_{1}, V_{2} \ldots V_{m}$ and hence the value of the contribution to the summation in eq 46 from the first term of each pair in the brace is equal to the area of a rectangle such as $a b c d$ (see $I I B^{\prime \prime}$ ) of width $s$ and of height equal to the height of the graph at point $P_{m}$. The second term of each pair contributes an amount equal to the area of a strip, also of width $s$, enclosed between

the axis of $y$ and the graph $e P_{m} f$ of $\left(\frac{V_{R}(y)}{V_{S}}-\frac{y}{b}\right) \sin \frac{k \pi y}{b}$. The last two terms of eq 46 correspond to the areas of the first and last half-sections and are in practice entirely negligible, because both the factors of the integrand are zero at $y=0$ and at $y=b$.

The approximate coefficients $G_{k}^{\prime}$ will therefore equal the true coefficients $G_{k}$ if the sum of the areas of the $n$ rectangles is equal to the total area between the curve and the axis. If the curve is reasonably smooth, as drawn in $I B^{\prime \prime}$ and $I I B^{\prime \prime}$, the error arising from lack of equality between the two types of strip is very small. Because of the rapid decrease of $C_{k}$ with increase in $k$, we are concerned only with the values of $G_{k}{ }^{\prime}$ for small values of $k$. For these the factor $\sin k \pi y / b$ changes but little over the narrow range, $s$, of any one strip, and also $y / b$ varies only by the small amount $1 /(n+1)$. Therefore, a significant error in any of the first few values of $G_{k}{ }^{\prime}$ is likely to arise only if the potential $V_{R}(y)$ changes so rapidly as to differ materially within each strip from its value $V_{m}$ at the beginning of each interval. Such a rapid change in $V_{R}(y)$ may occur if a ripple of wave length $b /(n+1)$ is present, and a consideration of this possibility is now in order.

Each plotted point in the curves of potential distribution such as figure 13 and figure 14 and of points such as $P_{m}$ of figure 24 , corresponds to the potential of the circle along which each conducting hoop, of circular section, is tangent to the imaginary cylindrical surface of radius $R$ considered in section $I X-1$. The insert at the upper right of figure 24 shows to scale for the normal $2-\mathrm{cm}$ spacing a cross section of two adjacent hoops and the points $P$ at which they are tangent to the surface $R R$. Therefore, in the immediate neighborhood of each point $P$, the potential must be nearly constant over a short distance and the curve of $V$ versus $y$ must have a horizontal tangent at each point. The actual shape of the curve is therefore similar to that shown in curve $C$ of figure 24, and has a rippled appearance. Here line $A$ shows again the ideal linear variation.

If we include in the Fourier series by which we attempt to represent $V_{R}(y)$, in addition to the $n$ terms of which the coefficients are the values of $G_{k}{ }^{\prime}$ already found, a term $G^{\prime}{ }_{2 n+2} \sin 2(n+1) \frac{\pi y}{b}$ and set $G^{\prime}{ }_{2 n+2}=-\frac{1}{2(n+1) \pi}$, this term will suffice to diminish the slope of the potential-distribution curve at each point of tangency by an amount equal to the mean slope $V_{S} / b$ and thus to give the rippled appearance. Moreover, the term itself vanishes at each of the points of tangency so that its inclusion does not disturb the fit of the series already found.

In section $I I C^{\prime}$ of figure 24 there is shown a ripple of this magnitude, and as shown at $I I C^{\prime \prime}$ its presence does not make the area $a^{\prime} e^{\prime} P_{m} f^{\prime} d^{\prime}$ materially different from the area $a^{\prime} b^{\prime} c^{\prime} d^{\prime}$. 
An exact meeting of the requirement for a horizontal tangent at each hoop can be obtained by including also for each of the $n$ terms of the original approximate series, such as the term $G_{p}^{\prime} \sin \frac{p \pi y}{b}$, a pair of compensating terms. These are

$$
-\frac{p G_{p}{ }^{\prime}}{4(n+1)} \sin (2 n+2-p) \frac{\pi y}{b} \text { and }-\frac{p G_{p}{ }^{\prime}}{4(n+1)} \sin (2 n+2+p) \frac{\pi y}{b} .
$$

The sum of such a pair of terms also vanishes at the points of tangency when $y=m b /(n+1)$, and therefore their inclusion does not affect the first $n$ values of $G_{k}^{\prime}$. Also the ripples which they introduce are in effect similar to those shown at $I I C^{\prime}$ in figure 24 and contribute on the whole substantially equal areas above and below the smooth curve $e^{\prime} P_{m} f^{\prime}$. Furthermore, the largest value of $p$ is equal to $n$, so that these terms, which must be introduced to fit the curve to the required horizontal tangent at each observed point, will range in order from $n+2$ to $3 n+2$. Table 3 shows that the values of $C_{k}$ corresponding to these high orders are so exceedingly small as to make the contribution of these terms to the correction $\Sigma C_{k} G_{k}{ }^{\prime}$ entirely negligible.

Unfortunately, ripples of the type just discussed and in such a phase as to satisfy the condition of horizontal tangency are not the only type which may occur.

A ripple of the same wave length, $b /(n+1)$, but occurring in such a phase that the points of tangency lie at or near one set of its crests, as shown at $D$ in figure 24 , will cause a cumulative systematic difference between the area under the curve and that of the rectangle and thus an error in the value of $G_{k}^{\prime}$ even for $k=1$.

A ripple of this latter type is to be expected if in the region external to the hoops there exists a component of electric field acting radially outward in addition to the normal axial field. If the electrometer is used at its full separation of $110 \mathrm{~cm}$ in the confined space available in the Bureau's present high-voltage laboratory, the grounded metal shields on the walkways are on the average at a distance of only $120 \mathrm{~cm}$ from the hoops. Hence the radial gradient near the uppermost hoops is nearly equal to the axial gradient. Under such conditions the potential at any point in the open spaces between the hoops will be lower than it would be if the radial component of field were absent.

In figure 24 distribution curve $D$ shows a ripple of this type and of about the magnitude probably present when the electrometer is used with the exaggerated hoop spacing of $6 \mathrm{~cm}$ (i. e., with two-thirds of the hoops removed). The area of the rectangular strip $a^{\prime \prime} b^{\prime \prime} c^{\prime \prime} d^{\prime \prime}$ is obviously not equal to the area $a^{\prime \prime} e^{\prime \prime} P_{m} f^{\prime \prime} d^{\prime \prime}$ between the curve and the axis and consequently by eq $46, G_{1}{ }^{\prime}$ is not equal to $G_{1}$.

A rough estimate of the magnitude of this shift in potential can be obtained from the treatment by Maxwell (Elec. and Mag. 1, sect. 203) for a plane grid of parallel wires, subjected to a field normal to the plane. This indicates that for an instrument of the present proportions this effect might introduce an error in voltage of 0.13 percent. It also indicates that the error would increase approximately as the square of the hoop spacing $s$, and for a spacing of $6 \mathrm{~cm}$ would amount to 1.4 percent. The experimental confirmation of the existence of these errors has already been discussed in section VI-8, page 284 . 


\section{VARIATION OF FORCE WITH HEIGHT OF DISK}

In the course of this work we have used as the best available basis for design and as a guide in reducing our observations, a theoretical expression for the attractive force based on the paper by Snow. ${ }^{26}$ In this paper there is given a solution for the electric field existing between an infinite plane conductor, simulating the lower plate, and two semiinfinite conductors which simulate the disk and the guard ring. These latter are spaced at distances $b$ and $c$, respectively, above the first conductor and have their lower surfaces plane and parallel to its upper surface. The two upper conductors are separated by a straight crevasse of width $a$ and of infinite length, the sides of which extend for an infinite distance upward perpendicular to the upper surface of the lower conductor. This crevasse simulates the gap between the disk and the guard ring. Snow's eq 27, when expressed in the present notation, is

$$
F_{s}=2 \pi w\left(\frac{V_{s}}{4 \pi c}\right)^{2}\left\{x-\frac{2 h}{\pi} \log _{e} \frac{2 c\left(e^{\frac{\pi x}{c}}-1\right)}{\left(a^{2}+h^{2}\right)^{1 / 2}}+\frac{a}{\pi} \tan ^{-1}\left(\frac{a}{h}\right)\right\}
$$

In this equation $F_{s}$ is the downward force acting on a rectangular strip of width $w$, extending back from the edge of the crevasse for a distance $x$, and $h$ is the height of the disk above the plane of the guard ring.

This can be arranged to give

$$
F_{s}=w \frac{V_{s}^{2}}{8 \pi c^{2}}\left\{x\left(1-\frac{2 h}{c}\right)-\frac{2 h}{\pi} \log _{e} \frac{2 c\left(1-e^{-\frac{\pi x}{c}}\right)}{\left(h^{2}+a^{2}\right)^{1 / 2}}+\frac{a}{\pi} \tan ^{-1}\left(\frac{a}{-h}\right)\right\}
$$

In this expression the first term in the brace contributes a force $\frac{V_{s}^{2} w}{8 \pi c^{2}}\left(1-\frac{2 h}{c}\right) x$, which is (neglecting terms in $h^{2} / c^{2}$ and higher) the same as $\frac{V_{s}^{2} w x}{8 \pi b^{2}}$. This contribution is proportional to the area of the strip and represents the normal force which would be produced on an area $w x$ by a uniform charge density $V_{s} / 4 \pi b$. The remainder of the righthand member of eq 48 must therefore be taken to represent the small correction per unit length due to the nonuniformity of charge density along the edge of one conductor.

This result may now be applied approximately to the actual case of a circular disk of radius $r_{d}$, by substituting its area $\pi r_{d}^{2}$ for the area $w x$ of the strip and its perimeter $2 \pi r_{d}$ for the width $w$ along the crevasse. By also substituting $r_{d}$ for $x$ in the exponential term of eq 48 we obtain the approximate relation

$$
F=\frac{V_{s}^{2}}{8 \pi c^{2}}\left[\pi r_{d}^{2}\left(1-\frac{2 h}{c}\right)-2 \pi r_{d}\left\{\frac{2 h}{\pi} \log _{e} \frac{2 c\left(1-e^{-\frac{\pi r_{d}}{c}}\right)}{\left(h^{2}+a^{2}\right)^{1 / 2}}-\frac{a}{\pi} \tan ^{-1}\left(\frac{a}{-h}\right)\right\}\right]
$$

26 Chester Snow. Effect of clearance and displacement of attracted disk, and also of a certain arrangement of conducting hoops, upon the constant of an electrometer. BS J. Research 1, 513 (1928) RP17. It may be noted that the method of applying this solution to the circular disk, which leads to his eq 29 , is incorrect. The process given here, which leads to eq 50 , has much greater justification but is still only an approximation. 
This can be reduced to

$$
F=\frac{V_{s}^{2} r_{d}^{2}}{8 c^{2}}\left[1+\frac{a}{r_{d}}-\frac{2 h}{c}-\frac{4 h}{\pi r_{d}} \log _{e} \frac{2 c\left(1-e^{-\frac{\pi r_{d}}{c}}\right)}{\left(h^{2}+a^{2}\right)^{1 / 2}}+\frac{2 a}{\pi r_{d}} \tan ^{-1}\left(\frac{h}{a}\right)\right]
$$

By introducing $r_{m}$, the mean of the radius of the disk and that of the aperture in the guard ring, that is

$$
r_{m}=r_{d}+\frac{a}{2}
$$

and neglecting terms in $\frac{a^{2}}{r_{d}^{2}}$, we have

and

$$
r_{m}^{2}=r_{d}^{2}\left(1+\frac{a}{r_{d}} \cdots \cdot\right)
$$

$$
F=\frac{V_{s}^{2} r_{m}^{2}}{8 c^{2}}\left[1-h\left\{\frac{2}{c}+\frac{4}{\pi r_{d}} \log _{e} \frac{2 c\left(1-e^{-\frac{\pi r_{d}}{c}}\right)}{\left(h^{2}+a^{2}\right)^{3 / 2}}-\frac{2 a}{\pi r_{d} h} \tan ^{-1}\left(\frac{h}{a}\right)\right\}\right]
$$

which is in the form

$$
F=\frac{V_{s}^{2} r_{m}^{2}}{8 c^{2}}[1-f h]
$$

where the coefficient $f$ varies only slightly with $h$, being in the form

where

$$
f=f_{0}+\frac{1}{r_{d}} \phi_{3}\left(\frac{h}{a}\right)
$$

$$
f_{0}=\frac{2}{c}+\frac{2}{\pi r_{a}}\left\{2 \log _{e}\left(\frac{2 c\left(1-e^{-\frac{\pi r_{d}}{c}}\right.}{a}\right)-1\right\}
$$

and

$$
\phi_{3}\left(\frac{h}{a}\right)=\frac{2}{\pi}\left\{\log _{e} \frac{1}{\left(1+\frac{h^{2}}{a^{2}}\right)}-\frac{a}{h} \tan ^{-1}\left(\frac{h}{a}\right)+1\right\}
$$

Figure 25 shows this quantity $f$ plotted as a function of $h$ for several different gap widths and spacings.

Equation 50 has proved to be of great utility in this work, but because of the approximations necessary in passing from the theoretical case, in which the field varies in two dimensions only, to the case of the actual electrometer, which is three-dimensional, it is not exact. The extent to which it correctly describes the variation of force with disk height is indicated in figure 26. This typical case is for the $16-\mathrm{cm}$ disk with a separation of $12 \mathrm{~cm}$, a clearance of $0.0106 \mathrm{~cm}$, and a mass $M_{F}$ of $2,550 \mathrm{mg}$. Here the abscissas are the scale readings of the electrometer. The disk and the guard ring are coplanar when the reading is $24.8 \mathrm{~cm}$, and differ in height by about $0.0625 \mathrm{~cm}$ at scale readings of $20 \mathrm{~cm}$ and of $30 \mathrm{~cm}$, the disk being high ( $h$ positive) for readings less than $24.8 \mathrm{~cm}$. 
If the electrometer is regarded as a deflection instrument, curve $B$, figure 26 , gives its observed scale law, each ordinate being the voltage corresponding to the scale reading plotted as abscissa. If the electrostatic force for a given voltage were independent of disk height, the variation in voltage would on the contrary be such as to produce the force needed to tilt the balance against the moment of the stabilizing weights. This force corresponds to curve $A$, the slope of which is $2 \gamma / M_{F}$. From curves $A$ and $B$ it is possible to compute for any scale reading what the electrostatic force at the corresponding disk height must be to give the scale law experimentally observed. This force is plotted in curve $C$, the force at the coplanar position being taken as unity. If the quantity $1-f h$ as calculated from eq 52 is subtracted

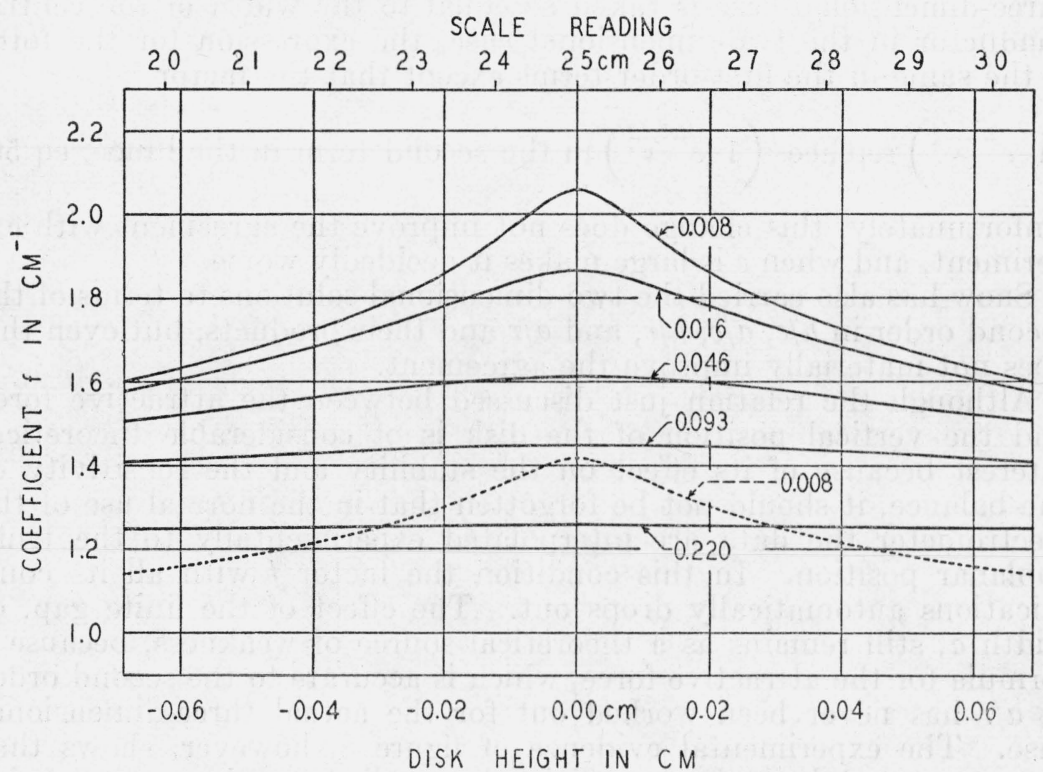

FIGURE 25.-Graphs showing computed values of the coefficient $f$ as a function of the height $h$ of the disk relative to the plane of the guard ring.

Solid-line curves are for 10-cm disk with different values of the width of gap ( $a$ in $\mathrm{cm}$ ) indicated on each curve. The dotted-line curve is for a 16-cm disk having the same gap width $(0.008 \mathrm{~cm})$ as the uppermost curve. Spacing $c=6 \mathrm{~cm}$ for all curves.

from curve $C$, the residuals are as shown in curve $D$. It is to be noted that because of the quadratic relation between force and voltage, the percentage discrepancies in terms of voltage here plotted to an enlarged scale in curve $E$ are only one-half of the corresponding differences in force shown in curve $D$. It will be seen that the residual force, not accounted for theoretically, is very small. At the $30-\mathrm{cm}$ point where the disk protrudes $0.0625 \mathrm{~cm}$ (six times the gap width) below the edge of the guard ring, the discrepancy is only 0.03 percent of the total force and only 0.4 percent of the actual change in force. At the 20-cm point, where the disk is withdrawn, the discrepancy is greater but the theory still accounts for 96 percent of the observed change in force. For wider separations the discrepancy becomes even less and is roughly inversely proportional to the separation. 
The difference between theory and experiment, though very small, is however definitely greater than the precision of the measurements, as is indicated by the close fit of the experimental points to the smooth curve $E$. There must be, therefore, a real discrepancy and it is probably introduced in the arbitrary process of passing to the actual threedimensional case from the two-dimensional case to which Snow's mathematical treatment of the field near the edge of the disk applies.

Later (unpublished) work by Snow has yielded a complete solution of another two-dimensional case in which the conductor which simulates the disk is bounded on two sides by infinitely long narrow crevasses which are bounded by the two semi-infinite conductors which simulate the guard ring. If the diameter of the disk in the three-dimensional case is taken as equal to the width of the central conductor in the two-dimensional case, the expression for the force is the same in the first-order terms except that the factor

$$
\left(1-e^{-\frac{2 \pi r_{d}}{c}}\right) \text { replaces }\left(1-e^{-\frac{\pi r_{d}}{c}}\right) \text { in the second term in the brace, eq } 50 .
$$

Unfortunately, this change does not improve the agreement with experiment, and when $c$ is large makes it decidedly worse.

Snow has also carried the two-dimensional solutions to terms of the second order in $h / c, a / c, h / r$, and $a / r$ and their products, but even this does not materially improve the agreement.

Although the relation just discussed between the attractive force and the vertical position of the disk is of considerable theoretical interest because of its effect on the stability and the sensitivity of the balance, it should not be forgotten that in the normal use of the electrometer the data are interpolated experimentally to the truly coplanar position. In this condition the factor $f$ with all its complications automatically drops out. The effect of the finite gap, of width $a$, still remains as a theoretical source of weakness, because a formula for the attractive force, which is accurate to the second order in $a / r$ has never been worked out for the actual three-dimensional case. The experimental evidence of figure 8 , however, shows that the gaps used have been sufficiently small to make second-order terms in $a / r$ negligible, and it is obvious that the first-order correction which we have used is that applying in the actual three-dimensional case.

\section{CONCLUSIONS AND DISCUSSION}

The absolute electrometer here described is intended for the accurate measurement of the effective value of alternating voltages up to 275,000 volts. It will be seen from the foregoing sections that for measurements up to 100,000 volts the accuracy approaches 0.01 percent.

The possibility of using the electrometer to make a new determination of the ratio, $v$, of the electrostatic to the electromagnetic unit of charge has been considered. For this purpose the spacing could be reduced to $12 \mathrm{~cm}$, at which value the hoops become unnecessary and the voltage gradient can be raised to $5,000 \mathrm{v} / \mathrm{cm}$. Using the $16-\mathrm{cm}$ disk, the attractive force would be $2.2 \mathrm{~g}$. Such a force could be weighed with sufficient accuracy if the optical magnification between the beam mirror and the scale were somewhat increased. It is 


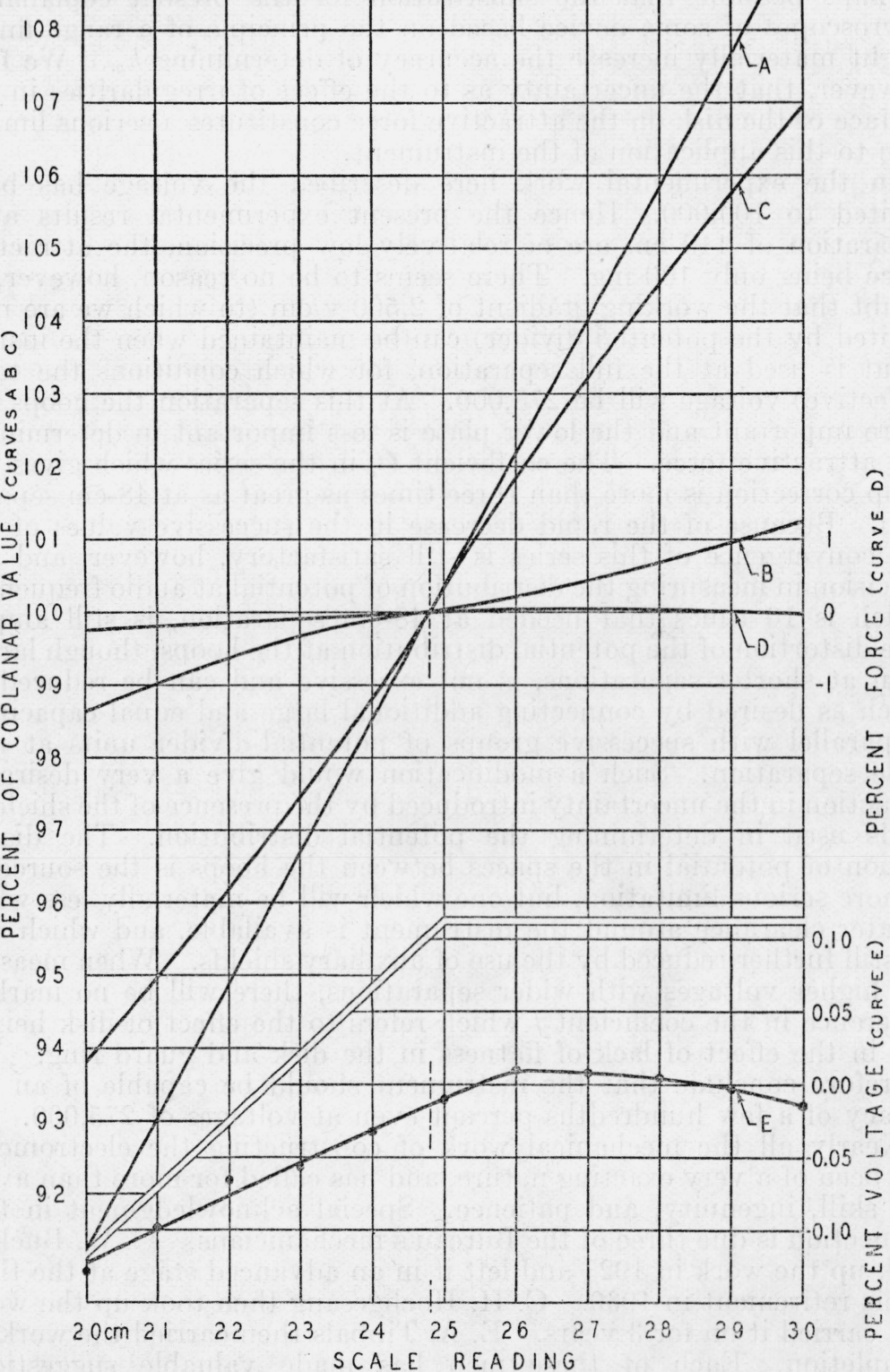

FIGURE 26.-Comparison of theoretical with experimental scale law for a 16-cm disk at 12-cm separation.

Abscissas are scale readings of the electrometer in centimeters and correspond to vertical displacements of the disk (protruding at 30 and receding at $20 \mathrm{~cm}$ ) from the coplanar position indicated by the dot-dash line. The ordinates of each curve are expressed as a percentage of the ordinate at the coplanar position. Ordinates of curve $A$ give the attractive force on the disk necessary to balance the resultant mechanical forces. For any given position of the disk this force is theoretically proportional to the square of the voltage. Ordinates of curve $B$ show for all positions on the scale the voltage experimentally found necessary for balance. Ordinates of curve $C$ were obtained by dividing the ordinates of curve $A$ by the square of the ordinates of curve $B$. Ordinates of curve $D$ were obtained by subtracting from the experimental curve $C$ the theoretical values of $(1-f h)$ calculated from eq 50. The residual curve $D$ has been replotted in curve $E$ to a much larger scale and in terms of voltage rather than of force. The fit of the individual points on this curve gives an indication of the high precision of the experimental results. 
perhaps possible that the substitution for the present coplanarity microscopes of some device based on the principle of a range finder might materially increase the accuracy of determining $h_{m}$. We feel, however, that the uncertainty as to the effect of irregularities in the surface of the disk on the attractive force constitutes a serious limitation to this application of the instrument.

In the experimental work here described the voltage has been limited to 100,000 . Hence the present experimental results at a separation of $110 \mathrm{~cm}$ are of relatively low precision, the attractive force being only $100 \mathrm{mg}$. There seems to be no reason, however, to doubt that the working gradient of $2,500 \mathrm{v} / \mathrm{cm}$ (to which we are now limited by the potential divider) can be maintained when the instrument is used at the full separation, for which conditions the total (effective) voltage will be 275,000 . At this separation the hoops are more important and the lower plate is less important in determining the attractive force. The coefficient $C_{1}$ in the series which gives the hoop correction is more than three times as great as at 48-cm separation. Because of the rapid decrease in the successive values of $G_{k}$, the convergence of this series is still satisfactory, however, and the precision in measuring the distribution of potential at audio frequency, which is 10 times that needed at 48-cm separation, is still ample. The distortion of the potential distribution at the hoops, though larger than at shorter separations, is not excessive and can be reduced as much as desired by connecting additional large and equal capacitors in parallel with successive groups of potential-divider units at this wide separation. Such a modification would give a very desirable reduction in the uncertainty introduced by the presence of the shielded leads used in determining the potential distribution. The distribution of potential in the spaces between the hoops is the source of a more serious limitation, but one which will be materially less when greater clearance around the instrument is available, and which can be still further reduced by the use of auxiliary shields. When measuring higher voltages with wider separations, there will be no marked difference in the coefficient $f$ which refers to the effect of disk height nor in the effect of lack of flatness in the disk and guard ring. We therefore conclude that the instrument should be capable of an accuracy of a few hundredths percent even at voltages of 275,000 .

Nearly all the mechanical work of constructing the electrometer has been of a very exacting nature, and has called for more than average skill, ingenuity, and patience. Special acknowledgment in this connection is due three of the Bureau's mechanicians. C. W. Buckey took up the work in 1923 and left it in an advanced stage at the time of his retirement in 1930 . C. H. Hochgesang then took up the work and carried it on for 3 years. E. A. Tibbals then carried the work to completion. Each of these men has made valuable suggestions relating to the design of important details.

The authors also express their appreciation of the hearty cooperation of the U. S. Naval Gun Factory authorities, and in particular of the personal attention given by Messrs. Crown of the foundry, Strauss and Fawcett of the metallurgical laboratory, and Murray of the torpedo tube shop. 
1. Disk.

Stainless-steel insert

4. Upper plate.

5. Surface gage.

. Gage block.

. Cover above disk.

Perforated plug in gage hole.

. Air duct.

.

. Prism. $a$

4. Suspension strip.

5. Weight lifter.

16. Weight pan.

17. Gold ligament

18. Beam mirror.

19. Fiducial mirror.

1. Chainomatic chain

. Stabilizing rod.

3. Balance colum

4. Balance base.

5. Balance-base legs.

26. Control rods. ${ }^{a}$

. Vernier reticle.

ctrometer scale

nd mounting.

1. Guard hoops

33. Gage rod.

4. Segmental gage block

5. Lower plate.

36. Silica pillars

3icarta inner pillars.

8. Invar rods

. Aluminum cross brace.

.

1. Jackscrews.

43. Base.

a In the actual instrument prism (13) is at $90^{\circ}$ from the position here drawn so that the reflected rays to the the lo rom those shown.

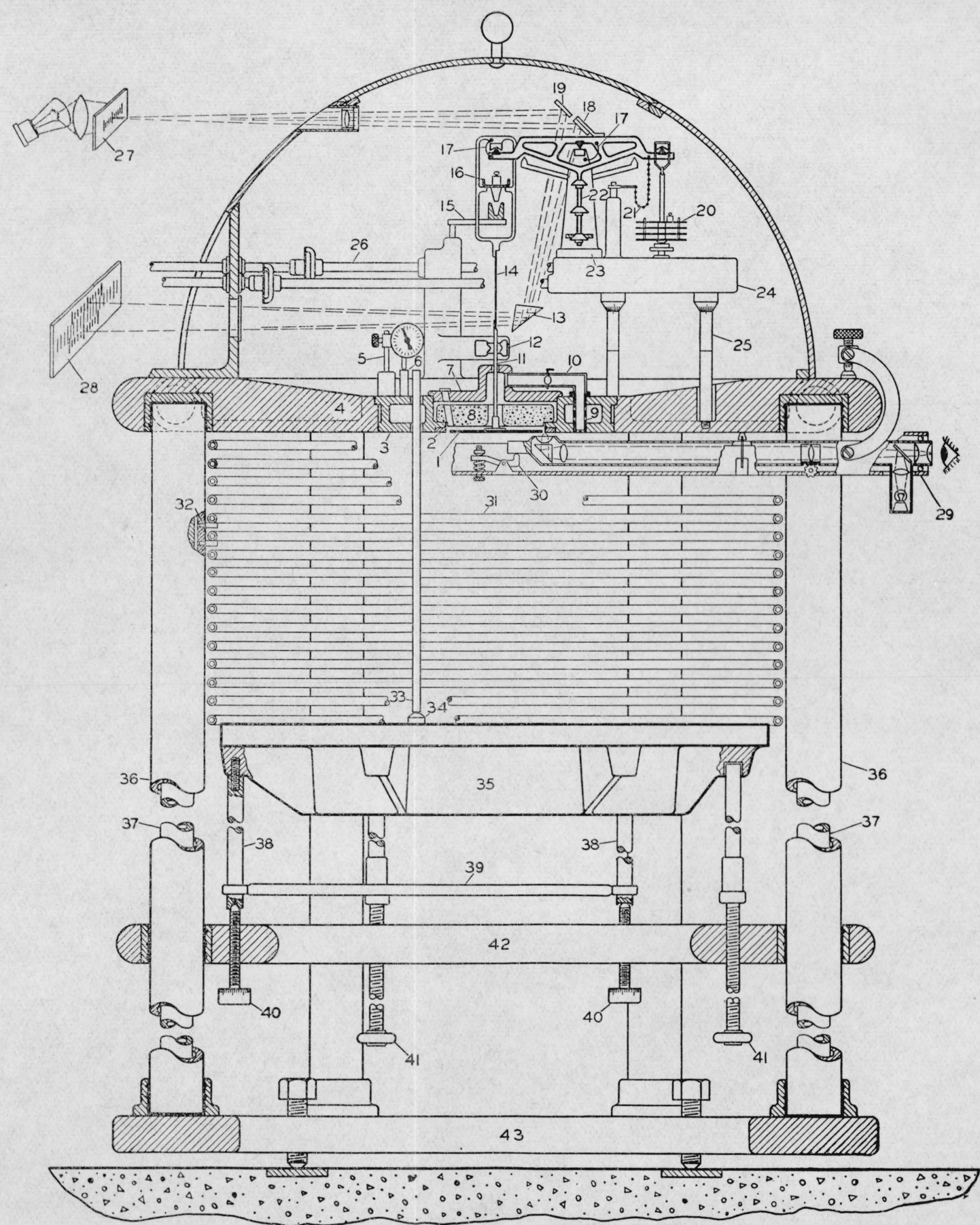

FIGURE 27.-Schematic cross section of electrometer and accessory equipment.

$44637-38$ (Face v. 314 ) 


\section{LIST OF PRINCIPAL SYMBOLS}

$A=$ area of disk.

$A_{0}=$ air force on disk when zero is read, dynes.

$A_{v}=$ air force on disk when voltage is measured, dynes.

$a=$ radial width of gap between disk and guard ring, centimeters.

$b=$ separation between disk and lower plate, centimeters.

$C_{k}=$ attenuation factor affecting Fourier coefficient of order $k$.

$c=$ separation between guard ring and lower plate, centimeters.

$F=$ attractive force on disk, dynes.

$F_{0}=$ attractive force on disk when disk and guard ring are coplanar, dynes.

$f=$ coefficient indicating change of force with height of disk, $\mathrm{cm}^{-1}$.

$f_{0}=$ value of $f$ when $h=0, \mathrm{~cm}^{-1}$.

$G_{k}=k$ th Fourier coefficient, defined by eq 14 , in the series describing the departure of the actual potential distribution from the ideal linear one.

$G_{k}{ }^{\prime}=$ coefficient approximating $G_{k}$ but derived by eq 17 from potential measurements at hoops only.

$g=$ acceleration of gravity, $980.08 \mathrm{~cm} \mathrm{sec}^{-2}$.

$h=$ height, $(b-c)$, of lower surface of the disk above that of guard ring, centimeters.

$h_{m}=$ value of $h$ when scale reading is $S_{m}$, and the coplanarity microscopes are used, centimeters.

$h_{d}=$ mean height of active surface of disk above the mean level of its rim, centimeters.

$i=\sqrt{-1}$.

$L=$ equivalent pointer length of optical system, centimeters.

$l_{c}, l_{d}, l_{t}=$ effective lengths of balance arms, centimeters.

$M_{r}=$ mass of removable weights used on disk arm of balance, grams.

$n=$ number of intermediate hoops.

$P=$ effective stiffness of balance, dyne-centimeters per radian.

$q=$ magnification of disk motion at scale, $=L / l_{d}$.

$R=$ radius of guard hoops, centimeters.

$r_{b}=$ radius of aperture in guard ring, centimeters.

$r_{d}=$ radius of disk, centimeters.

$r_{m}=$ mean of $r_{b}$ and $r_{d}$.

$r_{h}=$ radius of cross section of hoops, centimeters.

$S=$ reading on scale, centimeters.

$S_{m}=$ reading on scale when coplanarity microscopes are being used to determine $h_{m}$.

$S_{0}=$ zero scale reading with no voltage applied, centimeters.

$S_{0}=$ scale reading with voltage applied, centimeters.

$s=$ spacing of hoops, center-to-center, centimeters.

$V=$ potential with respect to that of the lower plate.

$V_{s}=$ voltage applied to electrometer in electrostatic cgs units.

$V_{M}=$ voltage applied to electrometer in electromagnetic volts (i. e., $10^{8} \mathrm{cgs}$ units).

$V_{m}=$ voltage of $m$ th hoop counting from the bottom.

$v=$ ratio of the electrostatic unit to the electromagnetic volt, $=$ 299.805 . 
$y=$ height above lower plate, centimeters.

$\gamma=$ stability coefficient, $\mathrm{g} \mathrm{cm} \mathrm{cm}^{-1}$.

$\Delta_{2}=$ second-order correction defined by eq 44 .

$\epsilon=$ dielectric constant of the air within the electrometer.

$\sigma=$ electric-charge density, electrostatic units.

$\phi_{1}=$ correction determined experimentally for effect of disk shape.

$\phi_{2}=$ correction determined experimentally for field distortion between hoops.

$\phi_{3}=\mathrm{a}$ function defined by eq 54 .

Washington, November 4, 1937. 